Aus der Klinik für Unfallchirurgie, Orthopädie und Plastische Chirurgie

(Prof. Dr. med. W. Lehmann)

der Medizinischen Fakultät der Universität Göttingen

\title{
Evaluation neuer sonographischer Bildverfahren im Schockraum zur \\ Erkennung von Thoraxverletzungen
}

\author{
INAUGURAL-DISSERTATION \\ zur Erlangung des Doktorgrades \\ der Medizinischen Fakultät der \\ Georg-August-Universität zu Göttingen
}

vorgelegt von

Söhren Dirk Brauns

aus

Kehl am Rhein

Göttingen 2020 
Dekan:

Betreuungsausschuss:

Betreuer:

\section{Prüfungskommission:}

Referent

Ko-Referent:

Drittreferentin:
Prof. Dr. med. W. Brück

Prof. Dr. med. K. Dresing

PD Dr. med. J. Uhlig

Prof. Dr. hum. biol. M. Schön

Datum der mündlichen Prüfung: 11.03 .2020 
Hiermit erkläre ich, die Dissertation mit dem Titel "Evaluation neuer sonographischer Bildverfahren im Schockraum zur Erkennung von Thoraxverletzungen" eigenständig angefertigt und keine anderen, als die von mir angegebenen Quellen und Hilfsmittel verwendet zu haben.

Kehl, den 02.08.2019 


\section{Inhaltsverzeichnis}

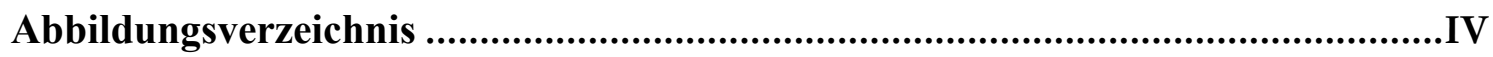

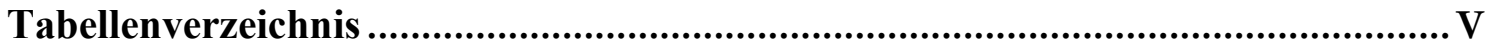

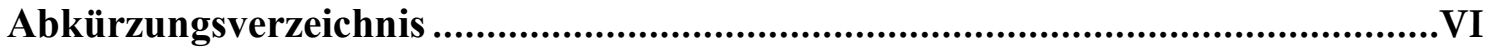

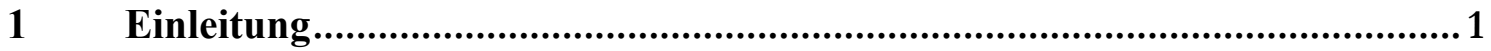

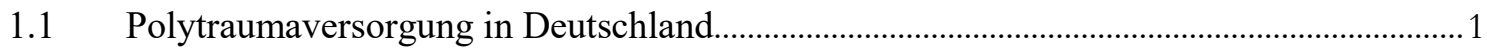

1.1.1 Skalen zur Einschätzung der Verletzungsschwere ...........................................................2

1.1.2 Organisation der Polytraumaversorgung in Deutschland......................................................6

1.1.3 Das überregionale Traumazentrum Göttingen-Kassel ........................................................

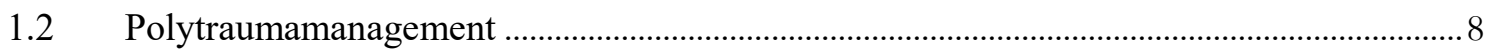

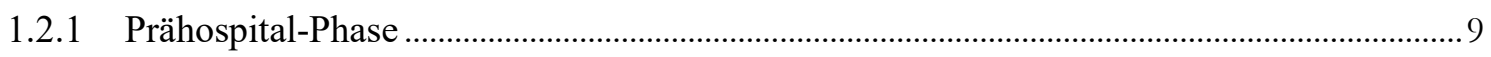

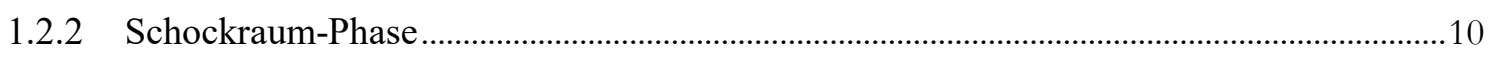

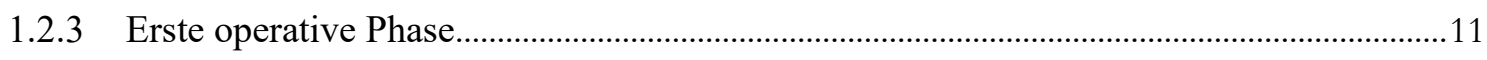

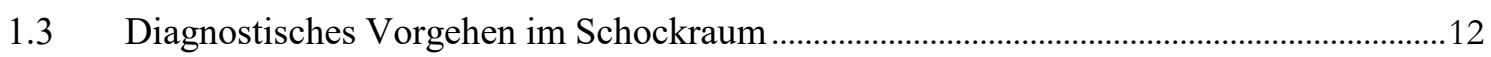

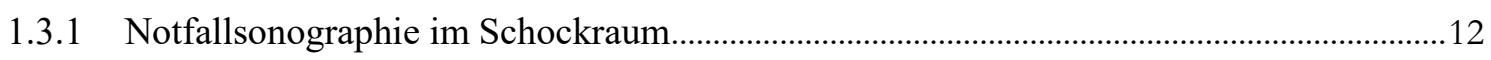

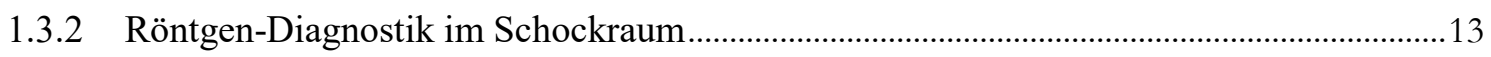

1.3.3 Computertomographie im Schockraum ……………………………...................................14

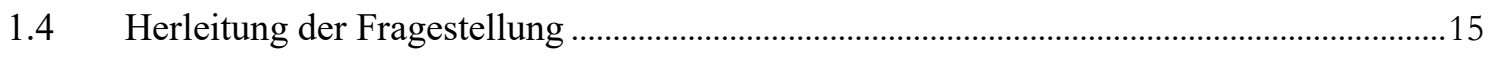

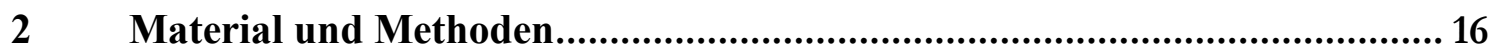

2.1 Beurteilung des Mediastinums im Schockraum mittels Thoraxröntgenuntersuchung .....16

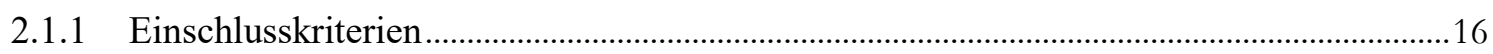

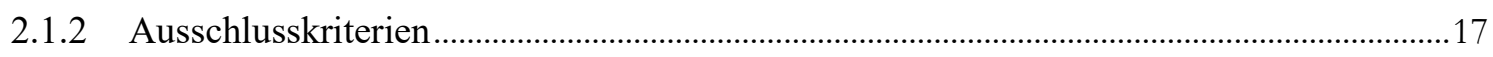

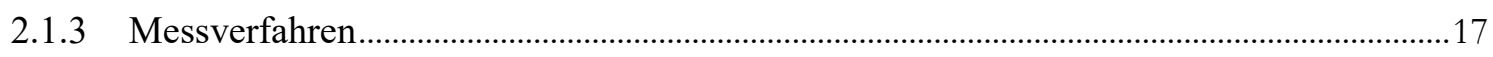

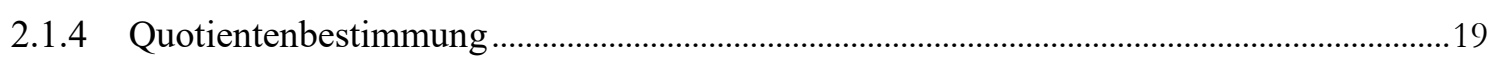

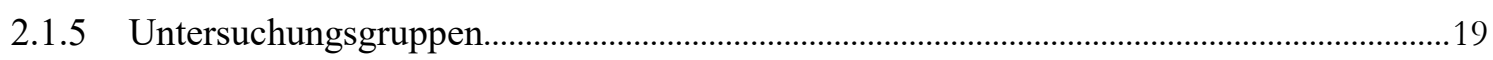

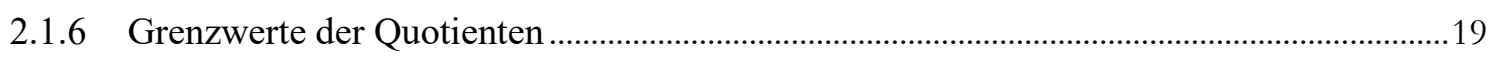

2.1.7 Analyseparameter zur Früherkennung mediastinaler Verletzungen ....................................20

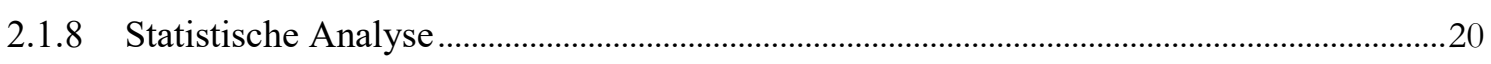

2.2 Erstdiagnostik eines Pneumo- und Hämatothorax durch eFAST und Thoraxröntgenuntersuchung im Schockraum .....................................................................22

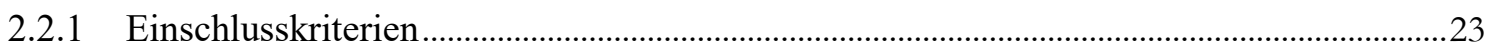

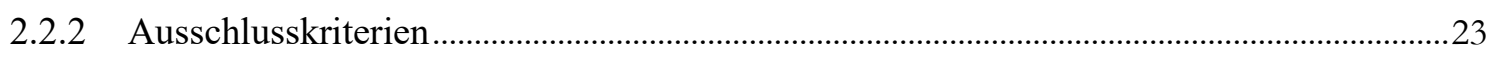

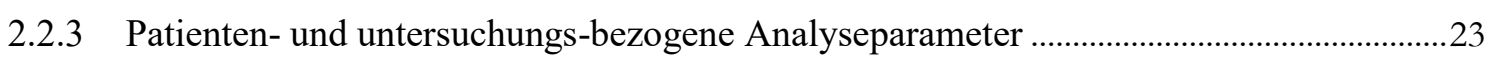

2.2.4 Statistische Auswertung der Testgenauigkeit von eFAST und
Thoraxröntgenuntersuchung ...........................................................................................23

2.2.5 Analyse der Konsequenzen aus eFAST und Thoraxröntgenuntersuchung .........................23 
2.3 Analyse von Thoraxröntgenuntersuchung und CT verstorbener

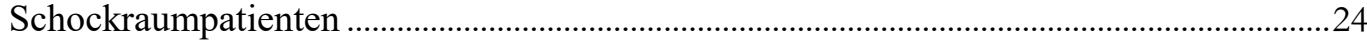

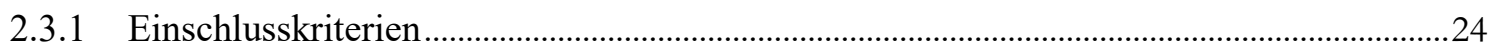

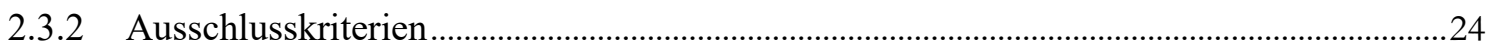

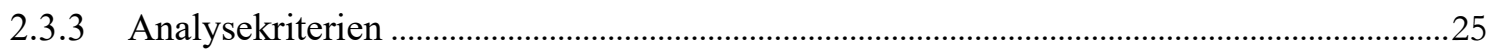

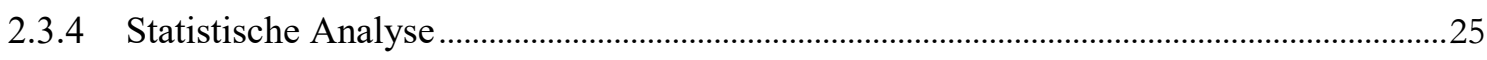

2.4 Modalitäten und Computerprogramme ……..................................................................2

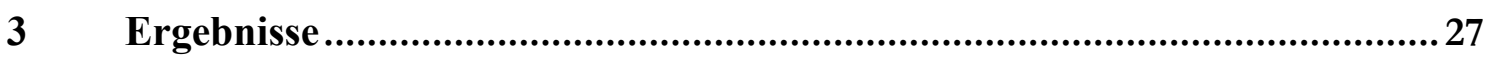

3.1 Die Thoraxröntgenuntersuchung zur Beurteilung des Mediastinums im Schockraum ....27

3.1.1 Mediastinum-Quotienten der Untersuchungsgruppen .........................................................29

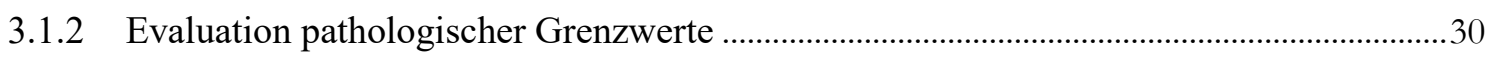

3.2 Früherkennung mediastinaler Verletzungen ........................................................................

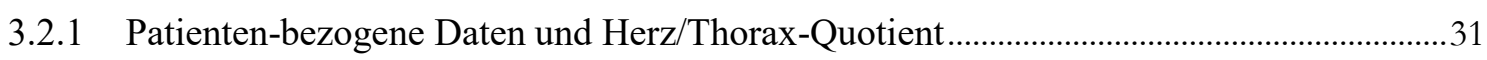

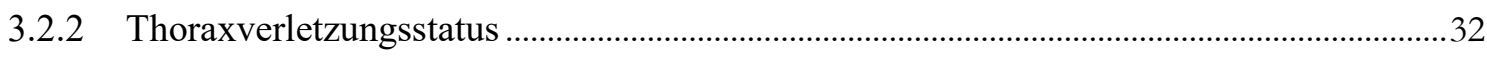

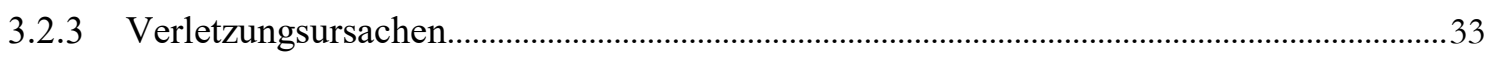

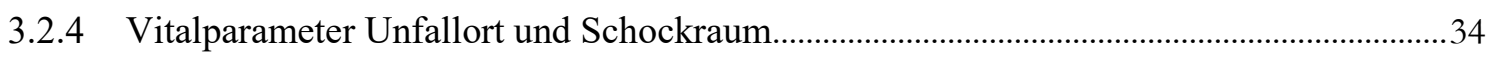

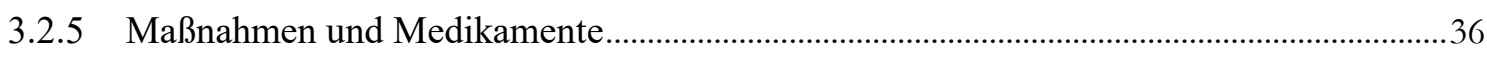

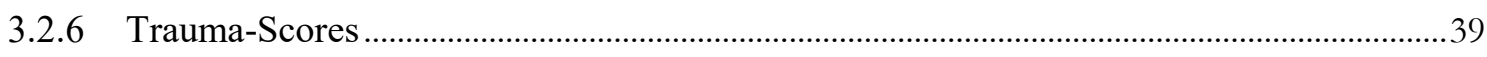

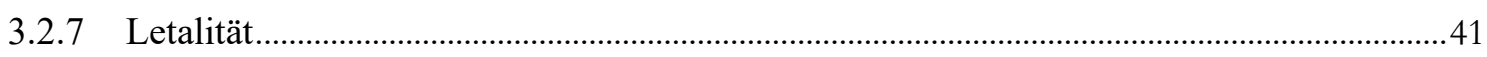

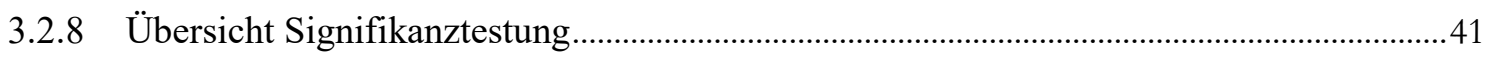

3.3 Vergleich bildgebender Verfahren der initialen Pneumo-/Hämatothorax-Diagnostik im Schockraum .................................................................................................................... 43

3.3.1 Testgenauigkeit von eFAST und Thoraxröntgenuntersuchung ................................................ 44

3.3.2 Konsequenzen aus eFAST und Thoraxröntgenuntersuchung ................................................ 45

3.4 Analyse von Thoraxröntgenuntersuchung und cCT/Ganzkörper-CT verstorbener

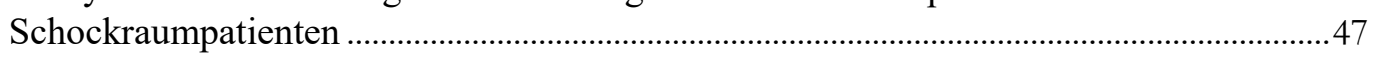

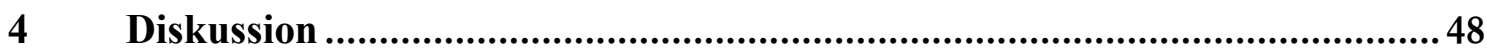

4.1 Die Rolle der Thoraxröntgenuntersuchung zur Beurteilung des Mediastinums im

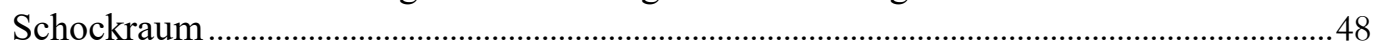

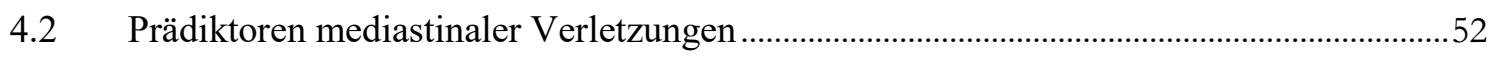

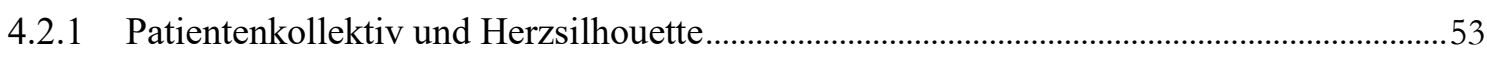

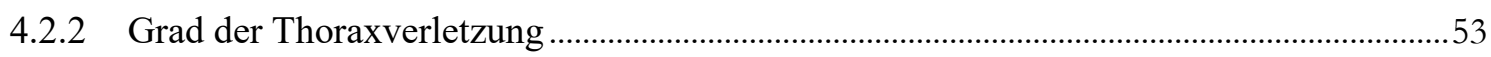

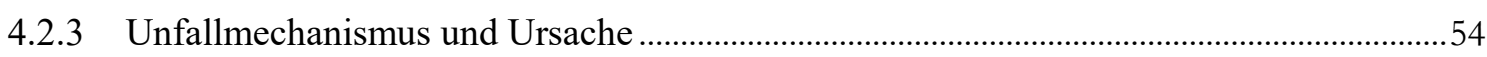

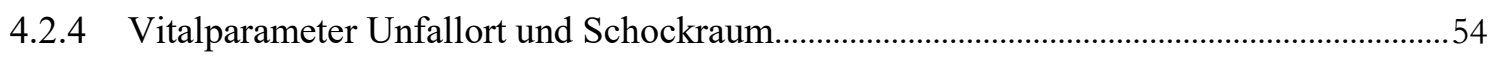

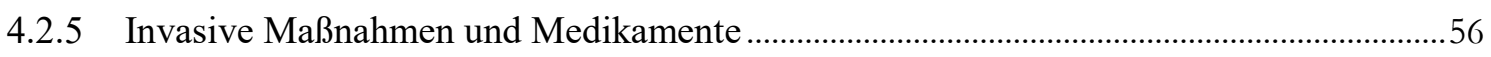

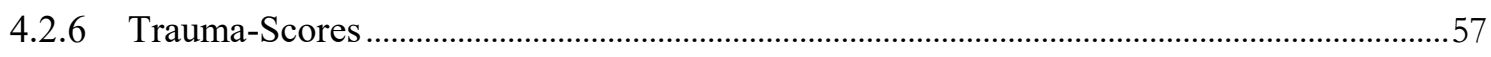

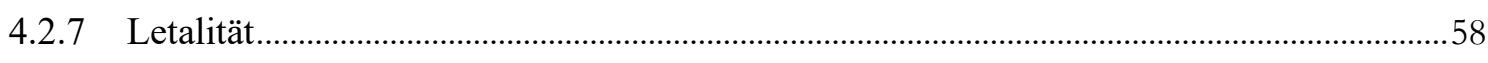

4.3 Aussagekraft von eFAST und Thoraxröntgenuntersuchung zur Pneumo- und

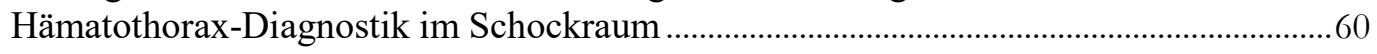

4.3.1 Konsequenzen aus eFAST, Thoraxröntgenuntersuchung und CT ……………………......63

4.4 Stellenwert von Thoraxröntgenuntersuchung und cCT/Ganzkörper-CT bei verstorbenen Schockraumpatienten. 


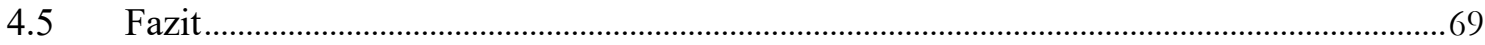

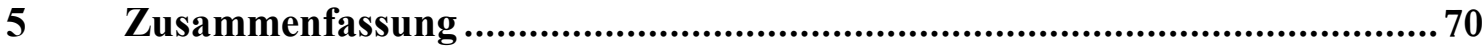

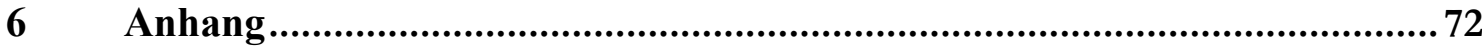

6.1 Initiale Beurteilung des Mediastinums mittels Thoraxröntgenuntersuchung im

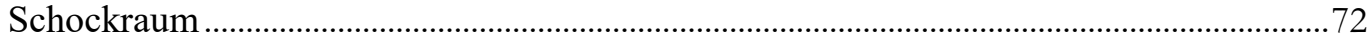

6.2 Früherkennung mediastinaler Verletzungen .............................................................................

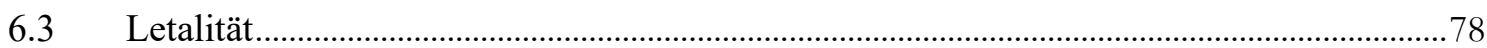

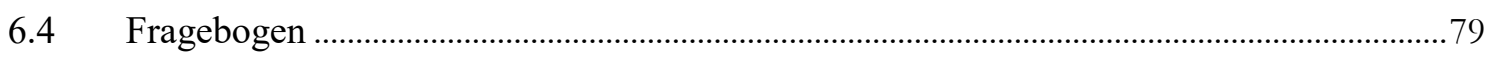

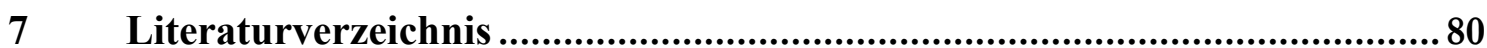




\section{Abbildungsverzeichnis}

Abbildung 1.1: Unfallursachen von Schockraumpatienten...........................................................2

Abbildung 1.2: Traumanetzwerk Göttingen-Kassel ...................................................................

Abbildung 1.3: Damage control und early total care ......................................................................

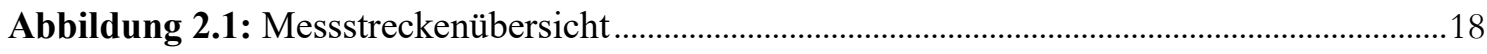

Abbildung 3.1: Messwertverteilung der Mediastinum/Thorax-Quotienten.....................................28

Abbildung 3.2: Geschlechterverteilung der Mediastinum/Thorax-Quotienten...............................28

Abbildung 3.3: Untersuchungsgruppen-bezogene Messwertverteilung der Mediastinum/Thorax-Quotienten.

Abbildung 3.4: Herzsilhouettengröße der Untersuchungsgruppen .....................................................32

Abbildung 3.5: Untersuchungsgruppen-bezogene Verletzungsschwere des Thorax .......................33

Abbildung 3.6: Thoraxverletzungsursachen der Untersuchungsgruppen........................................... 34

Abbildung 3.7: Systolische Blutdruckwerte der Untersuchungsgruppen.......................................... 35

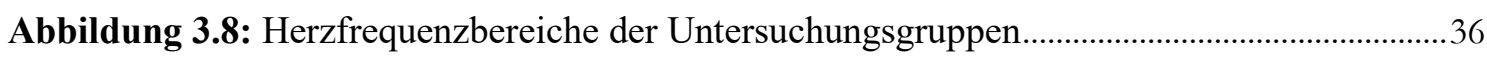

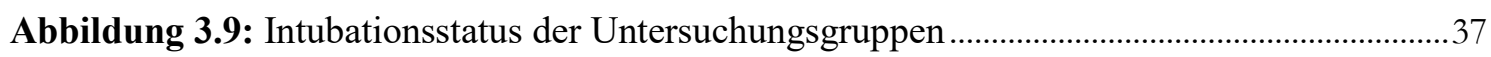

Abbildung 3.10: Thoraxdrainagenstatus der Untersuchungsgruppen ................................................ 38

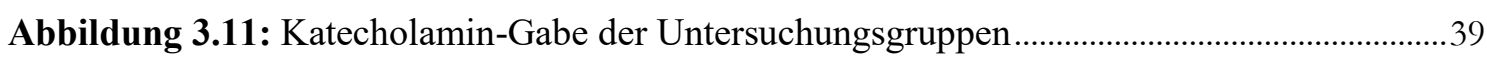

Abbildung 3.12: NISS-Punktwerte der Untersuchungsgruppen ...................................................... 40

Abbildung 3.13: RISC-II-Score der Untersuchungsgruppen ............................................................ 40

Abbildung 3.14: Prozentualer Letalitätsvergleich der Untersuchungsgruppen ................................ 41

Abbildung 3.15: Prozentuale Darstellung der eFAST-Befunde .......................................................... 44

Abbildung 3.16: Prozentuale Darstellung der Thoraxröntgenuntersuchungsbefunde.....................45

Abbildung 3.17: Prozentuale Verteilung der CT- und Thoraxröntgenuntersuchung ......................47

Abbildung 6.1: Fragebogen zur Pneumo-/Hämatothorax-Primärdiagnostik im Schockraum......79 


\section{Tabellenverzeichnis}

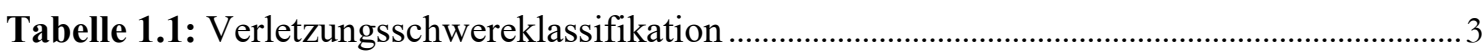

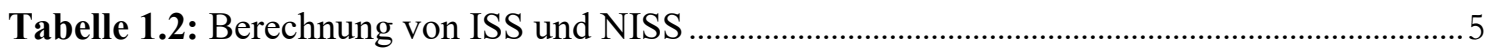

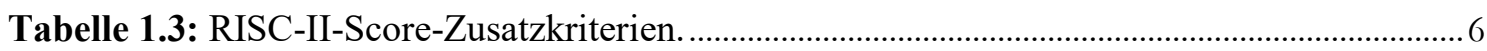

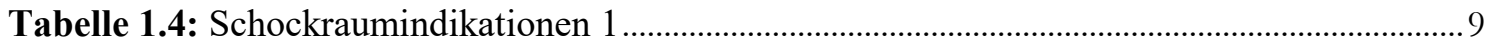

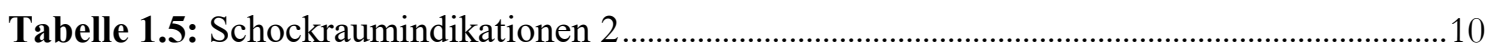

Tabelle 1.6: Sonographische Pneumothoraxzeichen ..................................................................13

Tabelle 2.1: Übersicht angewandter Signifikanztests Teil 1 .......................................................21

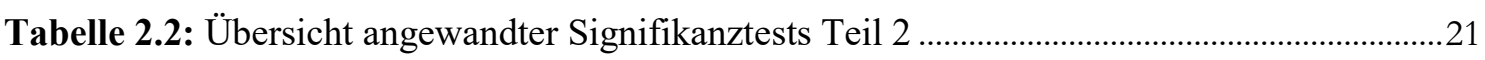

Tabelle 3.1: Definierte Grenzwerte des Mediastinum/Thorax-Quotienten Messwert 1................30

Tabelle 3.2: Definierte Grenzwerte des Mediastinum/Thorax-Quotienten Messwert 2................31

Tabelle 3.3: Ergebnisse der Signifikanztestung und Signifikanzniveau Teil 1 ................................ 41

Tabelle 3.4: Ergebnisse der Signifikanztestung und Signifikanzniveau Teil 2 ..............................42

Tabelle 3.5: Alters- und Geschlechterverteilung des Patientenkollektivs ........................................43

Tabelle 3.6: Prozentuale Testgenauigkeit von eFAST und Thoraxröntgenuntersuchung zur Pneumo-/Hämatothorax-Diagnostik im Schockraum.............................................................4

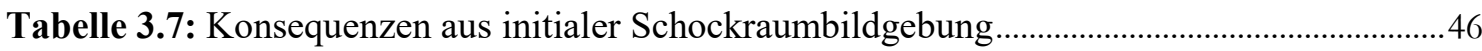

Tabelle 4.1: Autorenübersicht zur Testgenauigkeit der Thoraxröntgenuntersuchung in der Pneumo-/Hämatothorax-Diagnostik.

Tabelle 4.2: Autorenübersicht zur Testgenauigkeit von eFAST und Thoraxröntgenuntersuchung in der Pneumo-/Hämatothorax-Diagnostik......................................................63

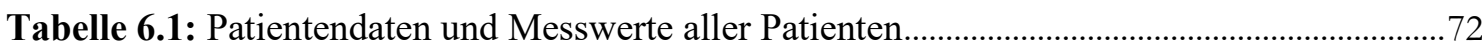

Tabelle 6.2: Grenzwert-bezogene Ergebnisverteilung des Mediastinum/Thorax-Quotienten

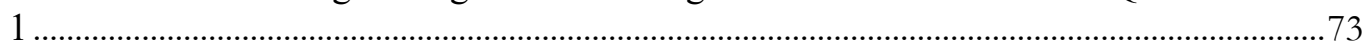

Tabelle 6.3: Grenzwert-bezogene Ergebnisverteilung des Mediastinum/Thorax-Quotienten

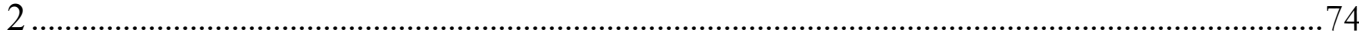

Tabelle 6.4: Übersicht deskriptive Daten des Herz/Thorax-Quotienten und der

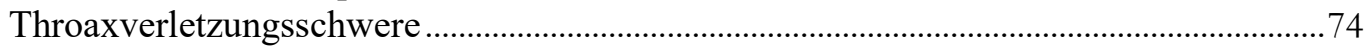

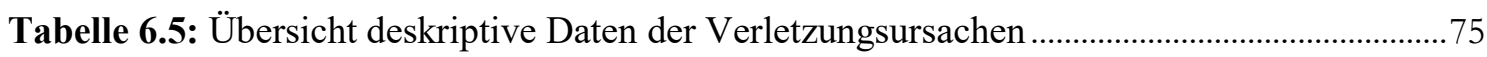

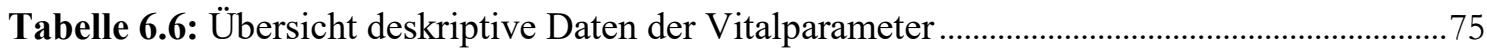

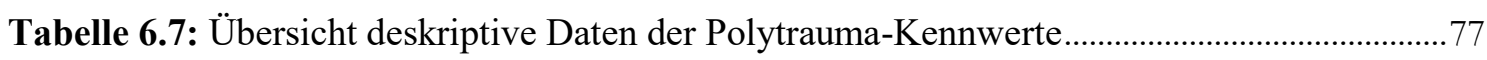

Tabelle 6.8: Übersicht deskriptive Daten zur Schockraumletalität...................................................78 


\section{Abkürzungsverzeichnis}

\begin{tabular}{|c|c|}
\hline AAST & American Association for the Surgery of Trauma \\
\hline AIS & Abbreviated Injury Scale \\
\hline ACS COT & American College of Surgeons Committee on Trauma \\
\hline ATLS & Advanced Trauma Life Support \\
\hline ARDS & Acute Respiratory Distress Syndrome \\
\hline CT & Computertomographie \\
\hline $\mathrm{DGU} \otimes$ & Deutsche Gesellschaft für Unfallchirurgie \\
\hline eFAST & Extended Focused Assessment with Sonography for Trauma \\
\hline ETC & European Trauma Course \\
\hline GCS & Glasgow Coma Scale \\
\hline Gruppe RöT & Gruppe Röntgenthorax \\
\hline Gruppe eF & Gruppe eFAST \\
\hline $\mathrm{HF}$ & Herzfrequenz \\
\hline ISS & Injury Severity Score \\
\hline Med./Thor.-Quotient & Mediastinum/Thorax-Quotient \\
\hline Neg. präd. Wert & Negativ prädiktiver Wert \\
\hline NISS & New Injury Severity Score \\
\hline $\mathrm{OP}$ & Operation \\
\hline PACS & Picture Archiving and Communication System \\
\hline PHTLS & Pre Hospital Trauma Life Support \\
\hline Pos. präd. Wert & Positiv prädiktiver Wert \\
\hline RISC /RISC-II & Riversed Injury Severity Classification/-II \\
\hline RR & Riva-Rocci (Blutdruck) \\
\hline SIRS & Systemic Inflammatory Response Syndrome \\
\hline SMR & Standard-Mortalitätsrate \\
\hline Sono & Sonographie \\
\hline $\mathrm{SpO}_{2}$ & Sauerstoffsättigung \\
\hline TRISS & Trauma and Injury Severity Score \\
\hline UMG & Universitätsmedizin Göttingen \\
\hline ZNS & Zentrales Nervensystem \\
\hline
\end{tabular}




\section{$1 \quad$ Einleitung}

\subsection{Polytraumaversorgung in Deutschland}

Im Jahr 2015 haben in Deutschland 9,73 Millionen Menschen eine Unfallverletzung und 24.578 einen tödlichen Unfall erlitten (BAuA 2017). Laut Jahresbericht des TraumaRegister DGU® (2018) wurden deutschlandweit 43.289 Schockraumpatienten ins Traumaregister aufgenommen. Davon hatten 18.805 Patienten einen ISS von $\geq 16$ und 4996 eine relevante Verletzung mindestens zweier Körperregionen, in Kombination mit einem physiologischen Problem (TraumaRegister DGUß 2018, Pape et al. 2014).

Das Polytrauma wurde 1987 von Tscherne definiert und bezeichnet die Verletzung von mehreren Körperregionen oder Organsystemen, wobei jede einzelne oder die Kombination mehrerer für den Betroffenen lebensbedrohlich ist. Die aktuelle Polytraumadefinition schließt zusätzlich hämodynamisch relevante und immunmodulatorische Vorgänge mit ein (Oestern et al. 2014, Niethard et al. 2014). Dies kann zum Systemic Inflammatory Response Syndrom (SIRS) und Multiorganversagen führen, weshalb polytraumatisierte Personen ein erhöhtes Morbiditäts- und Mortalitätsrisiko aufweisen (Butcher und Balogh 2009, Niethard et al. 2014).

Hauptursache von Polytraumen sind schwere Verkehrsunfälle und Absturztraumen. Sie stellen einen klinischen Notfall dar (ca. 1\% aller Notarzteinsätze) und führen in etwa 20\% zum Tod (Aschenbrenner und Biberthaler 2012).

Die führenden Unfallursachen der über den Schockraum eingelieferten Patienten, die als sogenanntes Basis-Kollektiv vom TraumaRegister DGU ${ }^{\circledR}$ zusammengefasst werden, sind in Abbildung 1.1 dargestellt. Dabei zeigt sich an erster Stelle der Sturz aus unter 3 m Höhe, an zweiter Stelle die PKW-Verkehrsunfälle, gefolgt von Stürzen aus über $3 \mathrm{~m}$ Höhe (siehe Abbildung 1.1). 


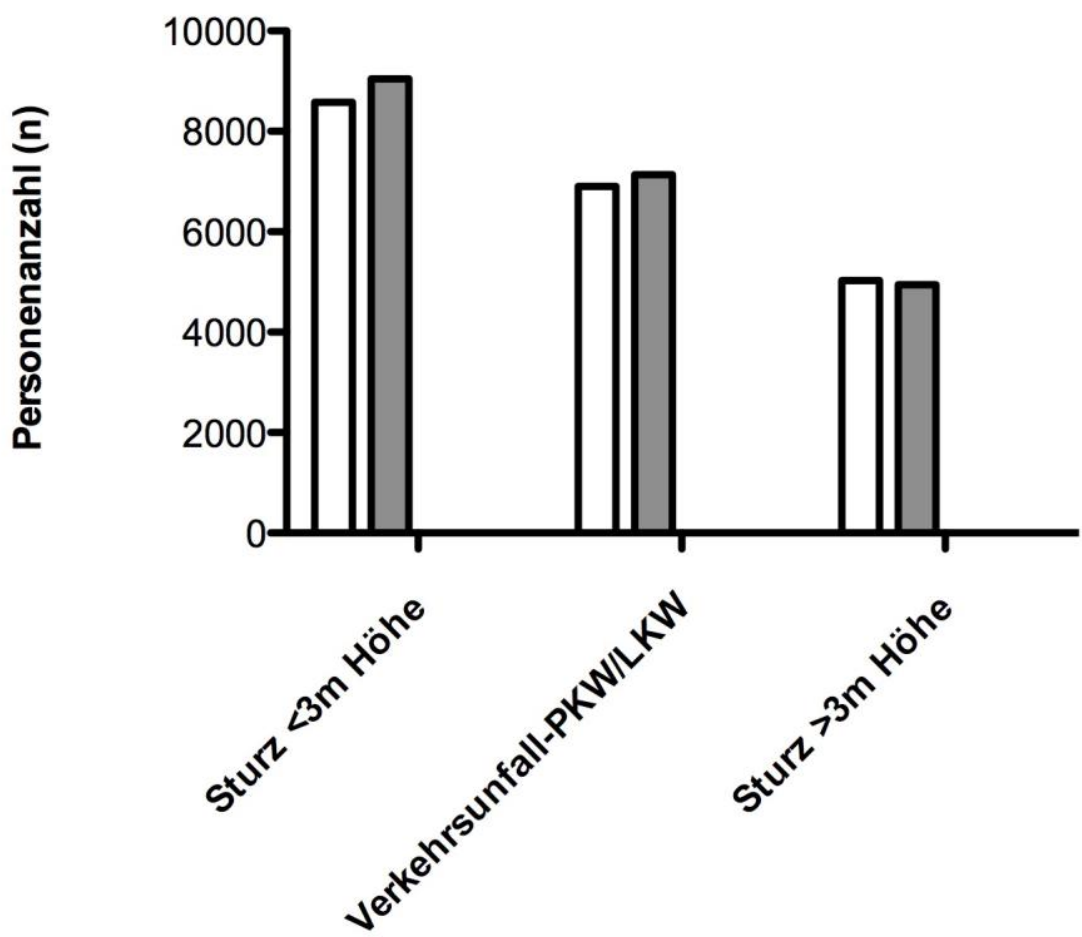

Abbildung 1.1: Graphische Darstellung der häufigsten Unfallursachen der deutschlandweit über den Schockraum eingelieferten Patienten (Basis-Kollektiv 2017/2018). Das weiße Säulendiagramm repräsentiert die Patientenanzahl des Basis-Kollektivs 2017 mit Sturz $<3 \mathrm{~m}$ Höhe $(\mathrm{n}=8583,26 \%)$, Verkehrsunfall-PWK/LKW $(\mathrm{n}=6907,21 \%)$ und Sturz $>3 \mathrm{~m}$ Höhe $(\mathrm{n}=5028$, 15\%). Grau hinterlegt wird die Patientenzahl des Basis-Kollektivs 2018 mit Sturz $<3 \mathrm{~m}$ Höhe $(\mathrm{n}=9042,26 \%)$, Verkehrsunfall-PWK/LKW $(\mathrm{n}=7134,21 \%)$ und Sturz $>3 \mathrm{~m}$ Höhe $(\mathrm{n}=4943$, $14 \%)$ dargestellt.

(modifiziert nach TraumaRegister DGU ${ }^{\circledR}$ 2017, TraumaRegister DGU® 2018)

Die hohe Sterblichkeit polytraumatisierter Patienten wird in eine frühe und eine späte Mortalität unterteilt. Die frühe Mortalitätsrate wird durch primäre Verletzungen des ZNS und traumatisch bedingten großen Blutverlust hervorgerufen (Keel und Trentz 2005, Meier und Habler 2007). Hierbei handelt es sich überwiegend um nicht mit dem Leben zu vereinbarende Verletzungen. Bei der späten Mortalität steht die Fehlregulation des Immunsystems im Vordergrund. Diese SIRS-vermittelte endotheliale Zellzerstörung kann durch Apoptose von Parenchymzellen zur Multiorgandysfunktion (MODS) bis hin zum Multiorganversagen (MOF) führen (Keel und Trentz 2005).

\subsubsection{Skalen zur Einschätzung der Verletzungsschwere}

Zur Vergleichbarkeit und zur Prognoseeinschätzung wurden verschiedene Scores entwickelt. Die Verletzungsschwere wird allgemein nach dem Injury Severity Score erfasst. 
1.1.1.1 Abbreviated Injury Scale (AIS)

Die Abbreviated Injury Scale dient als Grundlage zur Einteilung der Verletzungsschwere (Haasper et al. 2010). Sie ist ein Klassifikationssystem, basierend auf einer rein anatomischen Beschreibung der Verletzungen. Behandlungsart, -dauer oder -qualität werden nicht berücksichtigt (Baker et al. 1974, Haasper et al. 2010, Dokov und Radoinova 2014). Innerhalb der Körperregion wird die Verletzungsschwere in sechs Gruppen eingeteilt. Der AIS-Code 1 steht für unverletzte bzw. leichtverletzte Patienten, 6 für eine hohe Mortalitätswahrscheinlichkeit (Baker et al. 1974). Bei Personen mit Mehrfachverletzungen wird der höchste bzw. maximale AIS-Code (MAIS) aller verletzten Körperregionen herangezogen, um die allgemeine Verletzungsschwere zu klassifizieren (siehe Tabelle 1.1) (Haasper et al. 2010, Schmitt et al. 2010, Dokov und Radoinova 2014).

Das Besondere am AIS ist seine weitverbreitete Akzeptanz hinsichtlich der anatomischen Einteilung und Beschreibung der Überlebenswahrscheinlichkeit von Einzelverletzungen (Baker et al. 1974, States und Viano 1990). Europaweit dient der AIS als Grundlage für die offizielle Statistik zur Verletzungsschwereklassifikation und zur Definition lebensgefährlicher Verletzungen im Verkehr (DVR 2012).

Tabelle 1.1: Veranschaulichung der Verletzungsschwereklassifikation nach den Kriterien: Behandlungsart, MAIS und ISS.

\begin{tabular}{llcc}
\hline Verletzungsklassifikation: & Behandlungsart & MAIS & ISS \\
\hline Leichtverletzt & ambulant & $<2$ & $<5$ \\
Schwerverletzt & ambulant/stationär, & $2-4$ & $5-20$ \\
& ohne & & \\
& Intensivstation & & $>20$ \\
Schwerstverletzt & Stationär mit/ohne & $\geq 3$ & \\
& Intensivstation & & \\
\hline
\end{tabular}




\subsubsection{Injury Severity Score (ISS)}

Der ISS, erstmals 1974 von Baker publiziert, basiert auf der AIS-Klassifikation und beschreibt Verletzungen, die sowohl einzelne als auch mehrere Körperregionen betreffen (Lefering 2010). Es fließen der Anatomical Localizer (Schädel und Hals, Gesicht, Thorax, Abdomen, Extremitäten und Weichteile) und der Verletzungsgrad in die Berechnung ein, wodurch ISS-Werte von 1 bis maximal 75 Punkte ermittelt werden (Chawda et al. 2004). Punktwerte von 1 ergeben sich bei leichtverletzen, 75 bei schwerst- ggf. mehrfachverletzten Patienten. Laut Definition wird ein AIS-Code 6 nur vergeben, wenn die Verletzung einer Körperregion nicht mehr mit dem Leben zu vereinbaren ist, woraus automatisch ein ISS-Punktwert von 75 resultiert (Baker et al. 1974, Baker und O’Neill 1976). Der ISS korreliert sehr gut mit der Mortalitäts- und Morbiditätsrate sowie der Krankenhausverweildauer (Baker et al. 1974, Baker und O’neill 1976, Lefering 2002).

Die Berechnung des ISS erfolgt über die Summe der Quadratzahlen der AIS-Codes der drei am schwersten verletzten Körperregionen $\left(a^{2}+b^{2}+c^{2}=\right.$ ISS-Wert) (Copes et al. 1988, Baker et al. 1974).

\subsubsection{New Injury Severity Score (NISS)}

Bei der alten Definition des ISS konnten zeitgleich entstandene Mehrfachverletzungen wie beispielsweise eine Amputation an oberer und unterer Extremität nicht gleichzeitig berücksichtigt werden. Somit entstanden $\mathrm{zu}$ niedrige, nicht der tatsächlichen Verletzungsschwere entsprechende ISS-Werte. Deshalb wurde 1997 der ISS überarbeitet und erstmals als New Injury Severity Score publiziert (Osler et al. 1997). Dank der Überarbeitung konnte die zuvor angesprochene Schwäche durch Summation und Addition der drei höchsten AIS-Werte unabhängig von der Körperregion behoben werden (Osler et al. 1997, Whitaker et al. 2003). Zusätzlich zeigte sich beim NISS eine höhere Sensitivität und Spezifität bei polytraumatisierten Patienten mit multiplen Extremitätenverletzungen und eine andeutungsweise höhere Vorhersagekraft zu erwartender Komplikationen, verglichen mit dem ISS (Brenneman et al. 1998, Balogh et al. 2000, Husum und Strada 2002). 
Tabelle 1.2: Darstellung der Berechnung von ISS und NISS nach den sechs vom AIS definierten Körperregionen.

Score: $\quad$ AIS

Schädel und Hals

2

Gesicht

Thorax

Abdomen 3

Extremitäten

Untere: 4/Obere: 5

Weichteile 2

ISS $\quad(2)^{2}+(3)^{2}+(5)^{2}=38$ Punkte

NISS

$(3)^{2}+(4)^{2}+(5)^{2}=50$ Punkte

\subsubsection{Riversed Injury Severity Classification (RISC)-Score}

Der RISC-Score dient der Prognose der Überlebenswahrscheinlichkeit und wurde anhand von gesammelten TraumaRegister DGU® Daten (2800 Patienten) der Jahre 1993 bis 2000 entwickelt, validiert und löste 2003 den Trauma and Injury Severity Score (TRISS) ab (Lefering 2009). Der RISC-Score kombiniert zehn Informationen sowohl der anatomischen als auch der physiologischen Verletzungsschwere und stellt diese der standardisierten Mortalitätsrate gegenüber (Ruchholtz et al. 2008).

Im September 2014 wurde ein vom TraumaRegister DGU® erneut überarbeiteter Prognosewert, der RISC-II-Score, veröffentlicht. Zur Erhebung wurden mindestens mittelschwer verletzte Europäer (ISS $\geq 4$ ) der Jahre 2010 bis 2011 (insgesamt 30866 Patienten) analysiert und gegengeprüft. Das Ziel war, die in den letzten Jahren entstandene Abweichung zwischen standardisierter Mortalitätsrate und errechnetem RISC-Score durch vorher nicht berücksichtigte Beurteilungskriterien auszugleichen (siehe Tabelle 1.3) (Lefering et al. 2014). 
Tabelle 1.3: Darstellung der neuen RISC-II-Score-Zusatzkriterien.

RISC-II-Zusatzkriterien:

- Schwerste und zweitschwerste

Verletzung nach AIS-Klassifikation

- Unfallmechanismus

(stumpf/penetrierend)

- GCS (nur motorische Komponente)

- Kopfverletzungen
- Feinere Abstufung beim Alter

- Vorauffälligkeiten (ASA vor Unfall)

- INR anstatt Quick

- Pupillenreaktion und Pupillenweite

Ausgeschlossen: Alter $=0$; ISS $<4$

(modifiziert nach Lefering 2015)

\subsubsection{Einteilung von Thoraxverletzungen}

Thoraxverletzungen können anhand des Unfallmechanismus (stumpf oder penetrierend), anatomischer Strukturen (Brust-, Lungengewebe-, Atemwegs-, Ösophagus-, Diaphragma Verletzung) und definierter Schweregrade eingeteilt werden. Die Schweregrade reichen von Grad 0 (keine Thoraxverletzung) bis Grad III (schweres Thoraxtrauma) (Bölükbas et al. 2011, Khandhar et al. 2007, Blalock und Ochsner 1966). Die einzelnen Abstufungen werden anhand der Komplikationsraten und respiratorischen Einschränkung vorgenommen. So stehen bei Grad I Thoraxwandprellungen und einfache Rippen bzw. Sternumfrakturen im Vordergrund, die keine respiratorischen Einschränkungen oder Komplikationen verursachen. Bei Grad II (mittelschweres Thoraxtrauma) kommt es zu direkten Komplikationen und einer respiratorischen Insuffizienz. Bei Grad III (schweres Thoraxtrauma) liegt eine respiratorischen Insuffizient vor, die mittels Respiratortherapie behandelt werden muss (Encke et al. 1978).

\subsubsection{Organisation der Polytraumaversorgung in Deutschland}

\subsubsection{TraumaNetzwerk DGU®}

In Deutschland sind flächendeckend Traumazentren etabliert worden, die die Versorgung der Verletzten abdecken sollen. Mehrere Traumazentren haben sich zu sogenannten Traumanetzwerken zusammen geschlossen, die in den Bereichen Diagnostik, Therapie, Qualitätssicherung, Forschung, Fort- und Weiterbildung kooperieren (TraumaNetzwerk DGU ${ }^{\circledR}$ 2019a). Die teilnehmenden Kliniken werden anhand von definierten Kriterien, beispielsweise 24-stündige Facharztpräsenz 
verschiedener Abteilungen, Intensivkapazität, Verfügbarkeit personeller und apparativer Diagnostik, in lokale-, regionale- und überregionale Traumazentren unterteilt (Weißbuch DGU® 2006). Insgesamt nehmen 677 Kliniken aus Deutschland, Österreich, Schweiz, den Niederlanden, Belgien und Luxemburg teil, wodurch 53 zertifizierte, grenzüberschreitende Traumanetzwerke entstanden sind (TraumaNetzwerk DGU® 2019b, Sturm und Hoffmann 2016).

Die flächendeckende Telekooperation stellt einen weiteren Baustein des TraumaNetzwerks DGU ${ }^{\circledR}$ dar. Mit TKmed ${ }^{\circledR}$ entstand 2012 erstmals eine multidisziplinäre und sektorenübergreifende Vernetzung medizinischer Dienstleister, nach dem Jeder-mit-Jedem-Prinzip. Dies war die bislang einzige bundesweit verfügbare Teleradiologie- und Telekonsultationsplattform zur Bild- und Dokumentenübertragung (Sturm und Hoffmann 2016).

Das übergeordnete Ziel eines Traumanetzwerkes ist die Verbesserung der Versorgungsqualität von Schwerverletzten mittels optimierter Kommunikation, abgestimmter Versorgungsstandards und effizienter Nutzung regionaler und überregionaler Ressourcen (Ruchholtz et al. 2007, Ruchholtz et al. 2008).

\subsubsection{TraumaRegister DGU®}

Das TraumaRegister DGU® wurde 1993 als Instrument zur multizentrischen Erfassung von wissenschaftlichen, epidemiologischen und ökonomischen Behandlungsdaten Schwerverletzter im deutschsprachigen Raum gegründet (Nast-Kolb et al. 2000, Ruchholtz et al. 2008). Anhand der aktuellen Fall-, Behandlungs-, Operations- und Versorgungszahlen erfolgt die Klassifikation der einzelnen Kliniken (Neugebauer et al. 2012). Diese Prozessanalyse hilft den teilnehmenden Kliniken, Schwachstellen zu identifizieren, neue Behandlungskonzepte und Versorgungsabläufe zu überprüfen und ggf. zu etablieren (Ruchholtz et al. 2008).

\subsubsection{Das überregionale Traumazentrum Göttingen-Kassel}

Das überregionale Traumazentrum Göttingen-Kassel nimmt in Südniedersachsen und bundeslandübergreifend eine zentrale Rolle in der Versorgung schwerstverletzter Patienten ein. Die UMG zusammen mit dem Klinikum Kassel sind im Umkreis von ca. 150 Kilometer die größten Kliniken der unfallchirurgischen Maximalversorgung. In der UMG werden jährlich rund 800 Schwerverletzte im Schockraum der zentralen 
chirurgischen Notaufnahme behandelt, wovon mehr als 300 lebensbedrohlich (ISS $\geq 16$ ) verletzt sind (UMG 2019).

Das Traumazentrum Göttingen steht aufgrund der positiven Bilanz zwischen prognostizierter und tatsächlicher Letalität sowie der hohen Fallzahl im internationalen Vergleich an einer Spitzenposition (UMG 2019).

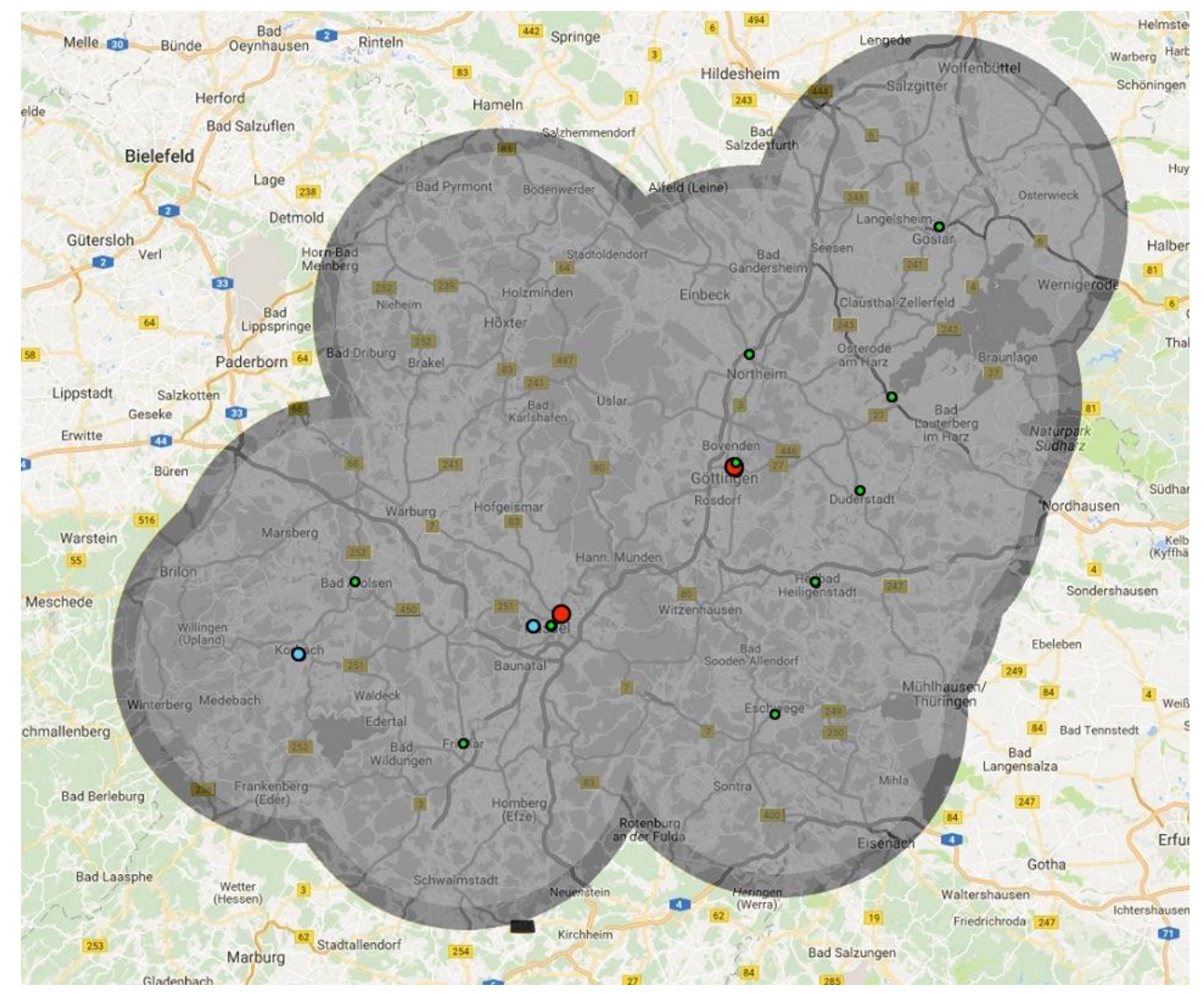

Abbildung 1.2: Einzugsgebiet des Traumanetzwerk Göttingen-Kassel, dargestellt als graue Fläche mit insgesamt 14 registrierten Kliniken. Das Universitätsklinikum Göttingen und das Klinikum Kassel (rote markierte Punkte) stellen überregional zertifizierte Versorgungszentren dar. Die blau und grün gekennzeichneten Städte stellen regional- und lokal zertifizierte Kliniken dar.

(modifiziert nach TeleKooperation TNW® 2019)

\subsection{Polytraumamanagement}

Das Polytraumamanagement erfolgt anhand von Prioritäten-orientierten Abläufen präklinisch durch das Pre Hospital Trauma Life Support (PHTLS), im Schockraum mittels Advanced Trauma Life Support (ATLS) und European Trauma Course (ETC) (Waydhas 2012). Der frühestmögliche präklinische Behandlungsbeginn und die schnellstmögliche Einleitung einer Kausaltherapie stellen übergeordnete Ziele dar 
(Fischer et al. 2016). Beim Polytraumamanagement werden verschiedene Behandlungsphasen unterteilt:

- Prähospital-Phase

- Schockraum-Phase

- Erste und zweite operative Phase

\subsubsection{Prähospital-Phase}

Die professionelle Behandlung des Patienten beginnt am Unfallort. Der Schwerpunkt liegt dabei laut aktueller Leitlinie Polytrauma (2016) nicht auf einem bestimmten Ablaufschema, vielmehr werden einzelne verletzungs-bezogene Aspekte fokussiert und ihren Prioritäten nach geordnet. Als Orientierungshilfen dienen Konzepte wie das PHTLS und übergreifende Betrachtungsweisen, wie die Empfehlungen der DGU ${ }^{\circledR}$ (Waydhas 2012, Leitlinie Polytrauma 2016). Es ist entscheidend, dass die Patienten präklinisch anhand ihrer Verletzungsschwere möglichst genau eingeteilt werden, um eine effiziente Schockraumversorgung $\mathrm{zu}$ gewährleisten und eine Unter- oder Übertriage möglichst zu vermeiden (Waydhas 2012). Als Anhaltspunkte zur präklinischen Beurteilung von Patienten dienen die in Tabelle 1.4 und Tabelle 1.5 aufgeführten Kriterien zur Schockraumalarmierung (Waydhas 2012).

Tabelle 1.4: Schockraumindikationen 1

Allgemeine Schockraumindikationen:

- Post-traumatisch: systolischer

Blutdruck $<90 \mathrm{mmHg}$

- Post-traumatisch: Atemstörung und Intubationspflicht

- Post-traumatisch: GCS $<9$

- Frakturen von $>$ als zwei proximalen Knochen

- Verbrennung von $20 \%$ und Grad $>2 b$
- Amputationsverletzung proximal von Händen/Füßen

- Penetrierende Verletzung der Rumpf-Hals Region

- Querschnittverletzung

- Offene Schädelverletzung

- Instabile Thorax-/Beckenfraktur

(modifiziert nach Neugebauer et al. 2012) 
Tabelle 1.5: Schockraumindikationen 2

Unfallmechanismus definierte Schockraumindikationen:

- $\quad$ Sturz aus $>3 \mathrm{~m}$

- Verkehrsunfall mit Frontalaufprall und Intrusion von $50-75 \mathrm{~cm}$

- Fußgänger- oder Zweiradkollision
- Tod oder Ejektion eines Autoinsassen

- Geschwindigkeitsänderung Delta $>30$ $\mathrm{km} / \mathrm{h}$

(modifiziert nach Neugebauer et al. 2012)

\subsubsection{Schockraum-Phase}

Die Patienten werden über einen Schockraum von medizinisch geschultem Fachpersonal aufgenommen. Wichtige Aspekte stellen 1. die Diagnostik, 2. das Erstellen eines adäquaten Therapiekonzeptes und 3. die Planung der Weiterversorgung dar. Zur adäquaten Schockraumversorgung werden interdisziplinäre Teams zusammengestellt, die schon vor der Ankunft des Schwerverletzten vollzählig anwesend sein sollten (Spering et al. 2017).

In überregionalen Traumazentren wird eine ressourcenorientierte Erweiterung des Schockraum Teams, je nach Verletzungsmuster und vitaler Bedrohung durchgeführt, um die Versorgungsdauer des Schwerverletzten reduzieren zu können (Spering et al. 2017, Piek und Jantzen 2000).

Im Schockraum selbst erfolgt die initiale Patientenbehandlung mittels primary und secondary survey. Dabei werden innerhalb der ersten Minuten nach Ankunft im Schockraum vital bedrohliche Zustände abgeklärt. Es wird ein der Situation und Verletzungsschwere angepasstes Handeln anhand des vom ATLS publizierten A(Airway), B-(Breathing), C-(Circulation), D-(Disability), E-(Exposure/Environment) Algorithmus angestrebt. Außerdem spielt bei der klinischen Untersuchung des Patienten die Bildgebung mittels Röntgen, CT und Notfallsonographie eine zentrale Rolle (Hokema et al. 2007). 


\subsubsection{Erste operative Phase}

Thoraxtraumen stellen die zweit häufigste Verletzung von Schockraumpatienten dar (TraumaRegister DGU® 2018). Deshalb ist es wichtig, vorliegende lebenslimitierende Thoraxverletzungen wie beispielsweise eine Aortenruptur, Aortendissektion oder Herzbeuteltamponade frühzeitig zu erkennen und ggf. notoperativ zu versorgen.

Bei der operativen Versorgung kann entweder nach dem early total care- oder dem damage control -Prinzip vorgegangen werden. Beim damage control -Prinzip werden Operationen auf die für den Patienten überlebensnotwendigen Schritte begrenzt (Doll et al. 2010). Das Ziel ist es, Sekundärschäden zu minimieren und das Outcome der Patienten zu maximieren (Bouillon et al. 2009). Die early total care zielt auf eine initial definitive Versorgung der Verletzung ab. Die Untersuchung von Pape H-C et al. (2009) zur Abgrenzung des damage control - und early total care -Prinzip zieht den im Schockraum vorliegenden Patientenzustand als Entscheidungskriterium heran (siehe Abbildung 1.3).

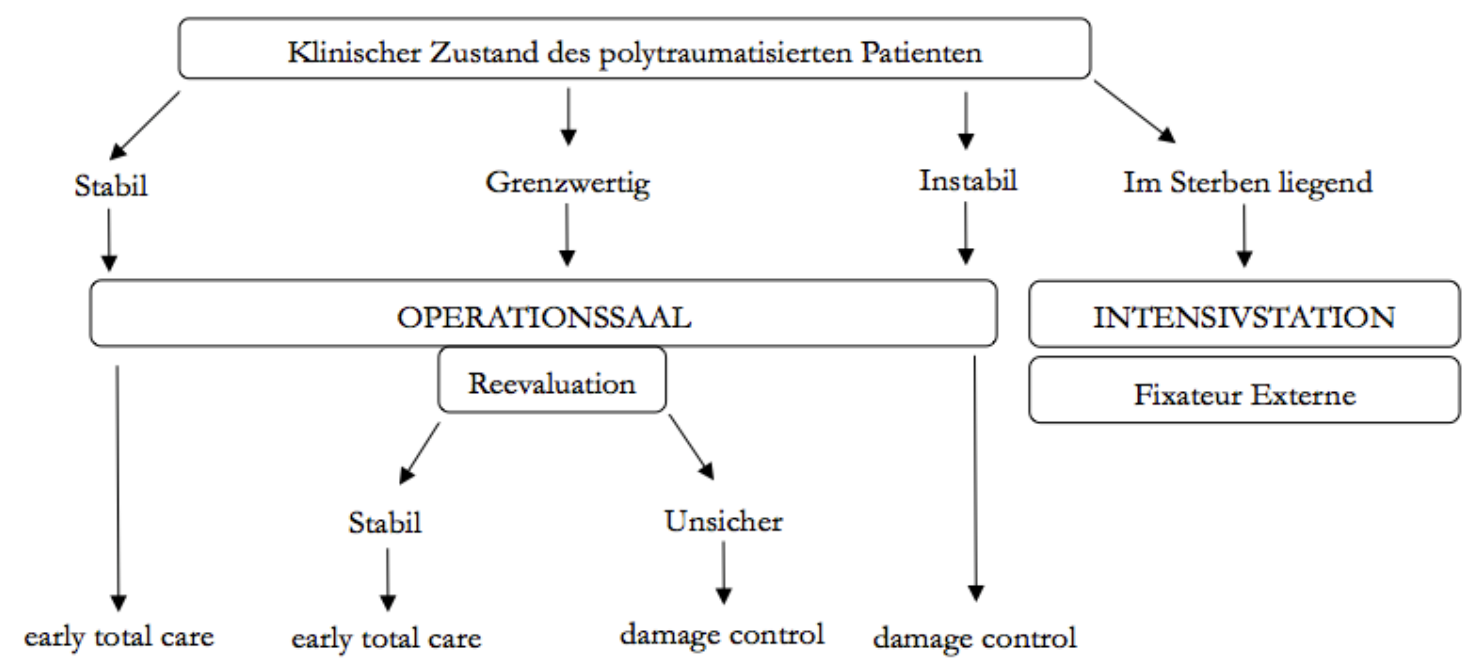

Abbildung 1.3: Damage control und early total care im Überblick. Einteilung der Behandlungsstrategien anhand des klinischen Zustandes des Schockraumpatienten (Stabil/Grenzwertig/Instabil/Im Sterben liegend) und angepasste Weiterbehandlung auf Intensivstation oder im Operationssaal. 


\subsection{Diagnostisches Vorgehen im Schockraum}

\subsubsection{Notfallsonographie im Schockraum}

Etabliert ist die Sonographie seit vielen Jahren im Schockraum zum Erkennen von lebensgefährlichen Blutungen. Die Sonographie ist eine nicht invasive, schnell durchführ- und erlernbare Untersuchung in Echtzeit mit hoher Auflösung und Verfügbarkeit (Koerner et al. 2008, Freeman 1999).

FAST bedeutet Focussed Asessment with Sonography for Trauma und stellt eine stark sensitive Methode zur Detektion freier Flüssigkeit im Abdomen dar (Lingawi und Buckley 2000). Freie intraabdominelle Flüssigkeit kann ab einem Volumen von etwa $100 \mathrm{ml}$ detektiert werden (Goldberg et al. 1970). Blutungsinduzierte freie Flüssigkeit im Abdomen führt alle 3 Minuten zu einem 1\%-igen Anstieg der Sterbewahrscheinlichkeit (Clarke et al. 2002). Deshalb hat das FAST einen hohen Stellenwert im Schockraum und hat sich als Standarduntersuchungsmethode bewährt (Leitlinie Polytrauma 2016).

Intraabdominelle Flüssigkeit verteilt sich entlang der Schwerkraft in die tiefsten Räume des Abdomens, dem Morison-Pouch (intraperitoneal im Bereich der rechten Niere), dem Koller-Pouch (intraperitoneal zwischen der Milz und der linken Niere) und dem Douglas-Raum (intraperitoneale Einsenkung zwischen Rektum und Uterus) (Koerner et al. 2008, Kirschning et al. 2009). Es gilt zu beachten, dass das FAST zwar eine hohe, Blutvolumen-abhängige Sensitivität zur Detektion freier Flüssigkeit besitzt, aber nur eine schwache Sensitivität zum Nachweis der ursächlichen Organverletzung (Koerner et al. 2008).

Thoraxtraumen stellen die zweit häufigste Unfallverletzungen von Schockraumpatienten dar (TraumaRegister DGU® 2018). Deshalb wurde eine Erweiterung (eFAST) der initial orientierenden Abdomensonographie (FAST) im Schockraum eingeführt, um unfall-assoziierte Krankheitsbilder im Thoraxbereich schnellstmöglich zu erkennen.

eFAST bedeutet Extended Focussed Asessment with Sonography for Trauma und zielt auf die Detektion freier Flüssigkeit im Thorax ab (Kirkpatrick et al. 2004, Mathis und Osterwalder 2010). Außerdem ermöglicht die Thoraxsonographie, bei fehlendem Lungengleiten einen Pneumothorax, bei echofreiem/echoarmem an das Zwerchfell grenzenden Raum einen Hämatothorax sowie Pleuraergüsse mit unterschiedlicher Sensitivität zu detektierten (siehe Tabelle 1.6) (von Ow und Osterwalder 2012, Kreuter et al. 2012). 
Das eFAST beinhaltet tiefe interkostal-, subxyphoidal-, subkostal, mediane Becken- und antero-laterale Thoraxschnitte (Wastl et al. 2015).

Tabelle 1.6: Sonographische Zeichen eines Pneumothorax

Pneumothoraxkriterien:

- Fehlendes Gleitzeichen/Power-slide Zeichen

- Fehlender Kometenschweifartefakt

- Nachweis von Wiederholungsechos
- Fehlende pulssynchrone Pleuraverschieblichkeit

- Fehlende präinterventionelle subpleurale Läsionen

- Nachweis des Lung point

(modifiziert nach Reissig und Kroegel 2006)

Limitierende Faktoren der Thoraxsonsographie stellen Emphyseme, schwere Adipositas und adhäsive Pleuraveränderungen dar (Kreuter et al. 2011, Reissig et al. 2011). Trotz steil verlaufender Lernkurve ist eine untersucher- und ausbildungs-abhängige Abweichung der Untersuchungsergebnisse und -qualität zu berücksichtigen (Potthoff et al. 2012, Rozycki und Shackford 1996, Shackford et al. 1999). Die Schallwellenundurchlässigkeit von Knochen und die Totalreflexion von Luft führen zu einer eingeschränkten diagnostischen Beurteilung von Frakturen und Lufteinschlüssen (Kreuter et al. 2012). Eine absolute Grenze der Notfallsonographie stellt die Eindringtiefe der Schallwellen dar. Sie kann bei adipösen Patienten aufgrund der großen Gewebemasse oberhalb der Organe eine Beurteilung stark erschweren (Grau 2007).

\subsubsection{Röntgen-Diagnostik im Schockraum}

Das Röntgen ist eine nicht invasive und seit Langem etablierte bildgebende Diagnostik im Schockraum (Körner et al. 2009). Körperregion- und Patientenkonstitutionsabhängig wird eine unterschiedliche Strahlungsqualität bzw. -stärke verwendet (Bundesärztekammer 2007). In der Vergangenheit war die Thoraxröntgenuntersuchung das Standardverfahren in der primären Schockraum-Diagnostik, wurde aber aktuell vom CT abgelöst (Leitlinie Polytrauma 2016).

Bei der Fragestellung Hämato-/ Pneumo- bzw. Spannungspneumothorax wird im Röntgenbild des Thorax nach sichtbaren Pleuralinien, einer vermehrten Transparenz 
und fehlender Gefäßzeichnung lateral der kollabierten Lunge gesucht (Traub et al. 2007, Diederich und Wormanns 2009).

Bei der Thoraxröntgenuntersuchung gilt laut Achenbach (2012), dass je kleiner, je weniger dicht und je zentraler eine Pathologie ist, umso leichter wird sie übersehen. Problematisch stellen sich außerdem technische Aspekte der Projektionsradiographie (Projektionsartefakte und Überlagerungsaspekte), die Schwierigkeiten einer orthograden Einstellung und die geringe Differenzierbarkeit geweblicher Aspekte dar. Darüber hinaus ist eine Darstellung intrakardialer Geschehen oder einer Lungenarterienembolie mittels Thoraxröntgenuntersuchung nicht möglich (Achenbach 2012).

\subsubsection{Computertomographie im Schockraum}

Die Computertomographie stellt den aktuellen Schockraumstandard zur radiologischen Untersuchung schwerverletzter Patienten dar (Leitlinie Polytrauma 2016). Die Computertomographie ist eine Schichtbildaufnahme, mit der sämtliche Gewebearten sowie Lage- und Formanomalien von Organstrukturen sehr genau differenziert werden können (Farkas 1986, Tsai et al. 2003).

Mit der Einführung des Multislice-CT konnte eine neue Dimension der Volumenerfassung, der Orts- und Zeitauflösung sowie eine 3D-Rekonstruktion anatomischer Strukturen ermöglicht werden (Schoepf et al. 1999, Flohr et al. 2007). Das Multislice-CT hat sich in der Schockraum-Diagnostik etabliert und dank kontinuierlicher Bilddatenakquisition, vorprogrammierter Untersuchungsprotokolle sowie schnellerer Bildberechnung zu einer Verbesserung der Schockraum-Diagnostik geführt, bei gleichzeitig signifikant verkürzter Untersuchungszeit (Novelline et al. 1999, Linsenmaier et al. 2001, Albrecht et al. 2004, Boehm et al. 2004).

Bei schwerverletzen Patienten kommt meist das Ganzkörper-CT (Schädel, Hals, Thorax, Abdomen und Becken) zum Einsatz, der sogenannte Traumascan. Hierbei können mittels 2D- und 3D-Rekonstruktionen Lungen-, Abdominal- und Gefäßverletzungen wie beispielsweise traumatische Aortenaneurysmen oder -rupturen detektiert werden (Rieger et al. 2002). Darüber hinaus können komplizierte Frakturen des Bewegungsapparates und intrazerebrale Ereignisse wie Blutungen oder Ischämien diagnostiziert werden (Schramm 2005). Außerdem stellt das CT die Untersuchungsmethode mit der höchsten Sensitivität und Spezifität zur Pneumo- und HämatothoraxDiagnostik dar (Reissig und Kroegel 2006). 
Bei der Gefäßdarstellung im CT gilt es zu beachten, dass die Kontrastmittelgabe ggf. mit Komplikationen, wie beispielsweise einer allergischen Reaktion, einer vermehrten Nierenbelastung oder einer thyreotoxische Krise einhergehen kann (Schönenberger et al. 2010). Außerdem geht das Multislice-CT mit einer um den Faktor 1,8 höheren Strahlenbelastung im Vergleich zum normalen Spiral-CT einher (Ruchholtz et al. 2002, Prokop et al. 2006).

\subsection{Herleitung der Fragestellung}

Die allgemeine Analyse diagnostisch-therapeutischer Behandlungen hat gezeigt, dass hierin ein großes Verbesserungspotential besteht (Messmer et al. 2001, Kanz et al. 2004, Ruchholtz 2004). Deshalb sollten die diagnostischen Handlungsabläufe anhand ihrer Therapierelevanz evaluiert und mittels Algorithmen zeitoptimiert vernetzt werden (Kanz et al. 1994, Ruchholtz et al. 1998).

Das Ziel dieser Arbeit ist es, zu klären:

- ob eine Optimierung des Schockraummanagements bei den bildgebenden Verfahren möglich ist;

- ob retrospektiv betrachtet, traumatisch bedingte Verletzungen des Mediastinums in der Thoraxröntgenuntersuchung z. B. durch die Bestimmung mediastinaler Quotienten erkannt werden können;

- ob eine Früherkennung mediastinaler Verletzungen anhand von präklinisch am Unfallort und klinisch im Schockraum erhobener Parameter möglich ist;

- ob sich aufgrund der Verletzung des Mediastinums ein Letalitätsunterschied zeigt;

- ob in einer 2. Phase anhand von prospektiv erhobener Parameter untersucht werden kann, ob Sonographie (eFAST) und Thoraxröntgenuntersuchung hinsichtlich der Erstdiagnose eines Pneumo-/Hämatothorax im Schockraum vergleichbar sind;

- ob sich aus einem der beiden Verfahren eFAST oder Thoraxröntgenuntersuchung mehr direkte therapeutische Konsequenzen, z. B. in Form einer Thoraxdrainage ergeben haben;

- ob sich retrospektiv betrachtet ein prozentualer Unterschied durchgeführter CT und Thoraxröntgenuntersuchungen bei im Schockraum verstorbenen Patienten zeigt;

- ob die ermittelten Daten durchgeführter CT- und Thoraxröntgenuntersuchungen verstorbener Schockraumpatienten konkordant oder abweichend sind von den im TraumaRegister DGUß dokumentierten Patienten. 


\section{Material und Methoden}

Die Arbeit umfasst eine Studie zur Erkennung von Thoraxverletzungen mittels bildgebender Verfahren im Schockraum der Universitätsmedizin Göttingen. Unter dem Aktenzeichen DOK_121_2016 wurde die Arbeit bei der Ethikkommission registriert.

\subsection{Beurteilung des Mediastinums im Schockraum mittels Thoraxröntgenuntersuchung}

Zur Ermittlung geeigneter Patienten wurde die Schockraumdokumentation 2013/2014 der UMG herangezogen. Alle aufgelisteten Patienten wurden hinsichtlich der Ein- und Ausschlusskriterien überprüft und pseudonymisiert in eine Excel-Tabelle übertragen.

Anschließend wurden alle relevanten Patienten aus dem PACS (Picture Archiving and Communication System) aufgerufen. Die a.p. Thoraxröntgenuntersuchung wurde an einem Befundungsmonitor begutachtet und, wie in Abschnitt 2.1.3 beschrieben, ausgemessen.

Die Identifizierung mediastinaler Verletzungen erfolgte mittels ICD-10 Kodierung und diente als Basis zur Einteilung der Patienten in zwei Untersuchungsgruppen: (1) Patienten mit mediastinaler Verletzung (Gruppe Auffällig) und (2) ohne Mediastinalverletzung (Gruppe Unauffällig).

Die statistische Auswertung erfolgte mittels Excel und Statistica, siehe Abschnitt 2.1.8. Zur Früherkennung mediastinaler Verletzungen erfolgte zusätzlich eine Untersuchungsgruppen-bezogene Signifikanztestung hinsichtlich am Unfallort (Zeitpunkt A) und im Schockraum (Zeitpunkt B) erhobener Parameter.

\subsubsection{Einschlusskriterien}

- Patienten, die im Zeitraum vom 01.01.2013 bis 31.12.2014 über den Schockraum der UMG aufgenommen wurden.

- Im Schockraum angefertigte Thoraxröntgenuntersuchung und CT-Untersuchung mit Abbildung des Thorax (CT-Thorax/Ganzkörper-CT/Traumascan).

- Patienten mit stumpfem Thoraxtrauma.

- Stör- und artefaktfreie Thoraxröntgenuntersuchung. 


\subsubsection{Ausschlusskriterien}

- Penetrierende Thoraxverletzungen.

- Thoraxröntgenuntersuchung mit Spineboard oder anderen Röntgenstrahlenbeeinflussenden Materialien.

- Andere Ein- oder Zuweisungswege als der Schockraum sowie elektiv für Operationen einbestellte Patienten.

Hinsichtlich Alter, Geschlecht und ethnischer Herkunft hat keine Limitierung stattgefunden.

\subsubsection{Messverfahren}

Es wurden sechs standardisierte Messstrecken gewählt, die im Folgenden beschrieben und in der Abbildung 2.1 als Übersicht dargestellt werden.

Unter Anwendung des Längenmesstools im PACS erfolgte die manuelle Bestimmung von Messstreckenansatz- und Endpunkt. Die ermittelten Entfernungen der angelegten Messpunkte wurden in der Maßeinheit Millimeter angegeben und in die pseudonymisierte Excel-Tabelle übertragen.

\section{Breitester Herzdurchmesser (Messstrecke a)}

Als breitester Herzdurchmesser wurde im Bereich der 6. bis 8. Rippe die Messstrecke a vom äußeren rechten Vorhofrand bis zum linken Ventrikelrand definiert (siehe Abbildung 2.1).

\section{Breitester Thoraxdurchmesser (Messstrecke b)}

Als breitester Thoraxdurchmesser bzw. Messstrecke b wurde die innere Thoraxapertur in Höhe der Messstrecke a definiert. Dabei wurde vom linken- bis zum rechten inneren Rippenrand der jeweiligen Thoraxseite gemessen (siehe Abbildung 2.1).

\section{Mediastinaldurchmesser 1 (Messstrecke A)}

Als Mediastinaldurchmesser 1 bzw. Messstrecke A wurde der Durchmesser der Aorta ascendens knapp oberhalb der Herzklappenebene definiert (siehe Abbildung 2.1). 


\section{Thoraxdurchmesser 1 (Messstrecke B)}

Als Thoraxdurchmesser 1 bzw. Messstrecke B wurde die äußere Thoraxapertur in Höhe der Messstrecke A definiert. Dabei wurde vom linken bis zum rechten äußeren Rippenrand gemessen (siehe Abbildung 2.1).

\section{Mediastinaldurchmesser 2 (Messstrecke A')}

Als Mediastinaldurchmessers 2 bzw. Messstrecke $A^{\prime}$ wurde die Breite des Mediastinums auf Höhe des Aortenbogens definiert (siehe Abbildung 2.1).

\section{Thoraxdurchmesser 2 (Messstrecke B')}

Als Thoraxdurchmesser 2 bzw. Messstrecke B' wurde die äußere Thoraxapertur in Höhe der Messstrecke A` definiert. Dabei wurde vom linken bis zum rechten äußeren Rippenrand gemessen (siehe Abbildung 2.1).

\section{Messstreckenübersicht}

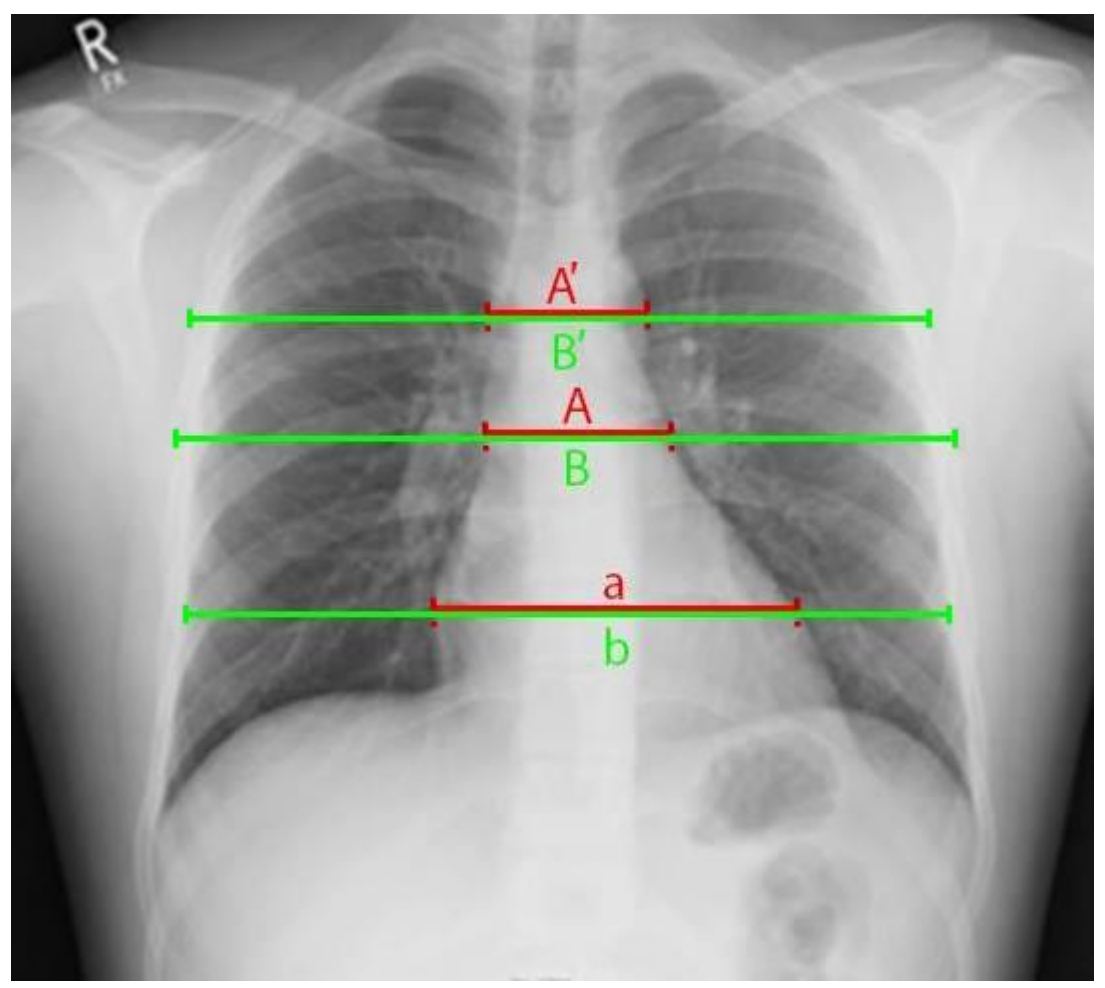

Abbildung 2.1: Thoraxröntgenuntersuchung mit Darstellung aller erhobenen Messstrecken mit Markierung der jeweiligen Messansatz- und Endpunkte. Die leicht höhenversetzte Darstellung der jeweils zueinander gehörigen Messstrecken ( $a-b, A-B$ und $\left.A^{\prime}-B^{\prime}\right)$ erfolgte zwecks besserer Veranschaulichung. 


\subsubsection{Quotientenbestimmung}

Der Herz/Thorax-Quotient ergibt sich aus der Messstrecke a (breitester Herzdurchmesser) und der Messstrecke b (breitester Thoraxdurchmesser).

Der Mediastinum/Thorax-Quotient Messwert 1 ergibt aus der Messstrecke A (Mediastinaldurchmesser 1) und der Messstrecke B (Thoraxdurchmesser 1).

Der Mediastinum/Thorax-Quotient Messwert 2 ergibt aus der Messstrecke A' (Mediastinaldurchmesser 2) und der Messstrecke B' (Thoraxdurchmesser 2).

Anschließend wurden alle ermittelten Quotienten in die pseudonymisierte Excel-Tabelle eingetragen.

\subsubsection{Untersuchungsgruppen}

Alle ausgemessenen Schockraumpatienten wurden anhand der ICD-10 Kodierung auf diagnostizierte Mediastinalverletzungen überprüft und als Auffällig bzw. Unauffällig in der pseudonymisierten Excel-Tabelle gekennzeichnet.

\subsubsection{Grenzwerte der Quotienten}

Die im Folgenden dargestellten Grenzwerte wurde in Anlehnung an die Untersuchung von Seltzer et al. (1981) gewählt:

1. Pathologisch vergrößertes Mediastinum $\geq 0,20$

2. Pathologisch vergrößertes Mediastinum $\geq 0,25$

3. Pathologisch vergrößertes Mediastinum $\geq 0,28$

4. Pathologisch vergrößertes Mediastinum $\geq 0,30$

Alle errechneten Mediastinum/Thorax-Quotienten wurden anhand der genannten Grenzwerte in Normalbefunde oder pathologische Befunde unterteilt. Anschließend erfolgte die Überprüfung der grenzwert-bezogenen Einteilung auf deren Reliabilität.

Die ermittelten richtig bzw. falsch positiven/negativen Ergebnisse wurden zur Berechnung der Sensitivität, Spezifität, des positiven- sowie des negativen prädiktiven Wertes herangezogen und mittels Statistica ausgewertet, siehe Abschnitt 2.1.8. 


\subsubsection{Analyseparameter zur Früherkennung mediastinaler Verletzungen}

Die Erhebung der Analyseparameter erfolgte durch die Auswertung der Schockraumprotokolle der Jahre 2013 und 2014. Die anschließende Signifikanztestung erfolgte nach Einteilung in die Patientengruppen Auffällig und Unauffällig.

Patientendaten

- Alter am Unfalltag, Geschlecht, Patientengruppe (Auffällig/Unauffällig), Grad der Thoraxverletzung (Grad 0 keine bis Grad III schwere Thoraxverletzung).

Messwerte

- Mediastinum/Thorax-Quotient: Messwert 1, Mediastinum/Thorax-Quotient: Messwert 2, Herz/Thorax-Quotient (normal/vergrößert).

Unfallart

- PKW, Sturz, Sonstige.

Unfallort und Schockraum

- Blutdruck (RR), Herzfrequenz (HF), Sauerstoffsättigung $\left(\mathrm{SpO}_{2}\right)$, Intubation (Ja/Nein), Katecholamin-Gabe (Ja/Nein), Thoraxdrainage (Ja/Nein).

Traumaparameter

- NISS, RISC-II Score.

Letalität

\subsubsection{Statistische Analyse}

Die statistische Auswertung erfolgte anhand pseudonymisierter Excel-Tabellen mittels Statistica.

Die deskriptive Statistik erfolgte anhand von Übersichtstabellen, die folgende Parameter berücksichtigen: Mittelwert, Minimum, Maximum, Standardabweichung, 1./3. Quantil und Ges.-Patientenanzahl (n).

Die explorative Datenanalyse erfolgte mittels Boxplot, Balken- und Kreisdiagramm. Ein signifikanter Unterschied der Untersuchungsgruppen wurde mit einem * gekennzeichnet.

Die Ermittlung der Spezifität, Sensitivität, des negativen und positiven prädiktiven Wertes erfolgte durch Einteilung der Ergebnisse in richtig bzw. falsch positiv und 
richtig bzw. falsch negativ. Die Berechnung der prozentualen Testgenauigkeit wurde anschließend mittels Statistica durchgeführt.

2.1.8.1 Variablen-bezogene Signifikanztestung und Anpassung des Signifikanzniveau Nach Überprüfung auf Normalverteilung und Kategorisierung anhand des Messniveaus wurden folgende Tests den einzelnen Variablen zugeordnet und mittels Statistica auf signifikante Unterschiede überprüft.

Tabelle 2.1: Übersicht analysierter Variablen und angewandter Signifikanztests des gesamten Patientenkollektivs.

\begin{tabular}{ll}
\hline Variablen & Signifikanztest \\
\hline Geschlechterverhältnis Med./Thor.-Quotient 1 & Mann-Whitney-U-Test \\
Geschlechterverhältnis Med./Thor.-Quotient 2 & Mann-Whitney-U-Test \\
Verhältnis Med./Thor.-Quotient Messwert 1 - Mann-Whitney-U-Test \\
Messwert 2
\end{tabular}

Eine Anpassung des ursprünglichen $p$-Wertes von $p \leq 0,05$ erfolgte mittels BonferroniMethode unter Berücksichtigung der drei durchgeführten Tests. Hieraus resultierte ein neues Signifikanzniveau von $\mathrm{p} \leq 0,017$.

Tabelle 2.2: Übersicht analysierter Variablen und angewandter Signifikanztests zur Früherkennung mediastinaler Verletzungen. Unterteilung und Testung des Patientenkollektivs anhand der Untersuchungsgruppen Auffällig und Unauffällig.

\begin{tabular}{ll}
\hline Variablen & Signifikanztest \\
\hline Geschlechterverhältnis & Pearson-Chi-Square-Test \\
Med./Thor.-Quotient 1 & Mann-Whitney-U-Test \\
Med./Thor.-Quotient 2 & Mann-Whitney-U-Test \\
Thoraxverletzung & Mann-Whitney-U-Test \\
RR Unfallort & Mann-Whitney-U-Test \\
$\mathrm{RR} \mathrm{Schockraum}$ & Mann-Whitney-U-Test \\
$\mathrm{SpO}_{2}$ Unfallort & Mann-Whitney-U-Test \\
$\mathrm{SpO}_{2}$ Schockraum & Mann-Whitney-U-Test
\end{tabular}




$\begin{array}{ll}\text { NISS } & \text { Mann-Whitney-U-Test } \\ \text { RISC-II-Score } & \text { Mann-Whitney-U-Test } \\ \text { Verletzungsursache } & \text { Fisher-Exact-Test } \\ \text { Intubation Unfallort } & \text { Fisher-Exact-Test } \\ \text { Intubation Schockraum } & \text { Fisher-Exact-Test } \\ \text { Thoraxdrainage Unfallort } & \text { Fisher-Exact-Test } \\ \text { Thoraxdrainage Schockraum } & \text { Fisher-Exact-Test } \\ \text { Katecholamine Unfallort } & \text { Fisher-Exact-Test } \\ \text { Katecholamine Schockraum } & \text { Fisher-Exact-Test } \\ \text { Herz/Thor.-Verhältnis } & \text { Fisher-Exact-Test } \\ \text { HF Unfallort } & \text { t-Test } \\ \text { HF Schockraum } & \text { t-Test } \\ \text { Letalität } & \text { t-Test }\end{array}$

Die Anpassung des ursprünglichen $p$-Wertes von $\mathrm{p} \leq 0,05$ erfolgte mittels BonferroniMethode unter Berücksichtigung der insgesamt 21 durchgeführten Tests. Hieraus resultierte ein neues Signifikanzniveau von $\mathrm{p} \leq 0,002381$.

\subsection{Erstdiagnostik eines Pneumo- und Hämatothorax durch eFAST und Thoraxröntgenuntersuchung im Schockraum}

Die Analyse der Pneumo-/Hämatothorax-Erstdiagnostik im Schockraum erfolgte mittels selbst erstellten Fragebogens, der im Anhang in Abbildung 6.1 eingesehen werden kann. Die Untersuchungsergebnisse aus eFAST und Thoraxröntgenuntersuchung wurden zusätzlich zur Schockraumdokumentation protokolliert und mittels Statistica ausgewertet, siehe Abschnitt 2.2.4.

Anschließend wurden in einem weiteren Schritt die auf dem Fragebogen vermerkten direkten Konsequenzen aus den jeweils vorangegangenen bildgebenden Verfahren (Thoraxröntgenuntersuchung/eFAST/CT) ausgewertet. 


\subsubsection{Einschlusskriterien}

- Patienten, die im Zeitraum vom 08.06.2015 bis 08.06.2016 über den Schockraum der UMG aufgenommen wurden.

- Im Schockraum durchgeführte Thoraxröntgenuntersuchung und/oder eFAST in Kombination mit CT (CT-Thorax/Ganzkörper-CT/Traumascan).

\subsubsection{Ausschlusskriterien}

- Patienten mit Hautemphysem.

- Penetrierende Thoraxverletzungen.

- Präklinische Anlage einer Thoraxdrainage.

- Inkomplette oder fehlerhafte Patientendokumentation.

\subsubsection{Patienten- und untersuchungs-bezogene Analyseparameter}

Die Erhebung der Analyseparameter erfolgte durch Auswertung des angefertigten Fragebogens:

Patientendaten

- Alter und Geschlecht.

Bestimmung der Testgenauigkeit

- Sensitivität, Spezifität, positiv und negativ prädiktiver Wert.

Prozentuale Gegenüberstellung der direkten Konsequenzen aus

- eFAST, Thoraxröntgenuntersuchung und CT.

\subsubsection{Statistische Auswertung der Testgenauigkeit von eFAST und Thoraxröntgenuntersuchung}

Die Differenzierung der Testgenauigkeit erfolgte durch Einteilung der Untersuchungsergebnisse in richtig bzw. falsch positiv und richtig bzw. falsch negativ. Anschließend wurde die Berechnung der Parameter Sensitivität, Spezifität, positiv und negativ prädiktiver Wert für Thoraxröntgenuntersuchung und eFAST mittels Statistica durchgeführt.

\subsubsection{Analyse der Konsequenzen aus eFAST und Thoraxröntgenuntersuchung}

Die Auswertung der untersuchungs-bedingten Konsequenzen erfolgte in tabellarischer Form anhand von folgenden Untersuchungsgruppen: 
- Gruppe eF 1 (eFAST und CT)

- Gruppe eF 2 (eFAST, Thoraxröntgenuntersuchung und CT)

- Gruppe RöT 1 (Thoraxröntgenuntersuchung und CT)

- Gruppe RöT 2 (Thoraxröntgenuntersuchung, eFAST und CT).

Sowie der Darstellung der Gesamtzahl durchgeführter Untersuchungen:

- eFAST

- Thoraxröntgenuntersuchung

- $\mathrm{CT}$.

Anschließend erfolgte für jede der zuvor genannten Untersuchungsgruppen die Darstellung der

- Anzahl aller untersuchten Personen (n);

- Anzahl untersuchungs-bedingter Konsequenzen;

- Gesamtzahl durchgeführter Konsequenzen.

In einem letzten Schritt erfolgte die prozentuale Berechnung der direkt aus eFAST, Thoraxröntgenuntersuchung und CT eingeleiteten Konsequenzen, gemessen an der Gesamtzahl aller durchgeführten Konsequenzen.

\subsection{Analyse von Thoraxröntgenuntersuchung und CT verstorbener Schockraumpatienten}

Die Detektion aller im Schockraum verstorbener Patienten erfolgte anhand der ICD-10 Kodierung. Alle relevanten Patienten wurden mittels PACS aufgerufen und die zu analysierenden bildgebenden Verfahren in einer pseudonymisierten Excel-Tabelle aufgelistet.

\subsubsection{Einschlusskriterien}

- Patienten, die im Zeitraum 01.01.2013 bis 31.12.2014 primär oder sekundär nach Verlegung über den Schockraum der UMG eingeliefert wurden.

- Patienten, die noch im Schockraum oder maximal 30 Tagen nach Aufnahme über den Schockraum in der Klinik verstorben sind.

- Thoraxröntgenuntersuchung oder CT im Schockraum der UMG.

\subsubsection{Ausschlusskriterien}

- Extern angefertigte Thorax röntgenuntersuchung oder CT. 
- Kein Bildmaterial im PACS.

\subsubsection{Analysekriterien}

Alle den Einschlusskriterien entsprechenden Patienten wurden hinsichtlich folgender durchgeführter bildgebender Untersuchungen analysiert:

- $\mathrm{CT}$ (cCT/Ganzkörper-CT/Traumascan)

- Thoraxröntgenuntersuchung.

\subsubsection{Statistische Analyse}

Die patienten-bezogenen Daten (Geschlecht und Durchschnittsalter) wurden zur deskriptiven Analyse des Patientenkollektivs herangezogen.

Anschließend wurde die prozentuale Verteilung im Schockraum durchgeführter Thoraxröntgenuntersuchung und $\mathrm{CT}$ (cCT/Ganzkörper-CT/Traumascan) einander gegenübergestellt.

\subsection{Modalitäten und Computerprogramme}

\section{Röntgen}

Fabrikat: Siemens, Ysio Systemversion VB10 (Healthcare Siemens, Erlangen Deutschland).

\section{Computertomographie}

Fabrikat: Siemens, Somatom AS Plus (Healthcare Siemens, Erlangen Deutschland).

\section{Sonographie}

Fabrikat: Siemens XCLASS, AcusonX300 premium Edition (Siemens Medical Solutions, California USA).

\section{Computerprogramme}

- Graphpad Prism 5 for MAC OS X, Version 5 (La Jolla, CA USA).

- Microsoft Excel for MAC 2011, Version 14.3.4 (130416), Update 14.3.4 (Microsoft, Redmond, Washington USA). 
- PACS-Software GE Healthcare.

- $\quad$ STATISTICA, Version 12.7 (StatSoft, Tulsa, Oklahoma USA). 


\section{Ergebnisse}

\subsection{Die Thoraxröntgenuntersuchung zur Beurteilung des Mediastinums im Schockraum}

Das Patientenkollektiv bestand aus 267 Patienten. Insgesamt wurden 205 männliche und 62 weibliche Patienten im Alter von 0 bis 92 Jahren und einem Altersdurchschnitt von $49 \pm 21$ Jahren analysiert. Aufgeteilt in die Untersuchungsgruppen zeigten sich 27 Patienten mit mediastinaler Verletzung (Gruppe Auffällig) und 240 Patienten ohne mediastinale Verletzung (Gruppe Unauffällig). Von den 27 auffälligen Patienten waren 23 männlich und vier weiblich. Von den 240 als unauffällig befundeten Patienten waren 182 männlich und 58 weiblich. Sämtliche erhobenen Mediastinalverhältnisse der Untersuchungsgruppen wurden hinsichtlich der Parameter Mittelwert, Standardabweichung, Gesamtanzahl (n), 1. und 3. Quantil analysiert und im Anhang in Tabelle 6.1 aufgeführt.

\section{Geschlecht- und Messwertanalyse}

Initial wurden alle erhobenen Mediastinum/Thorax-Quotienten (Messwert 1 und 2) einander gegenübergestellt. Hierbei zeigte sich ein Durchschnittswert für den Messwert

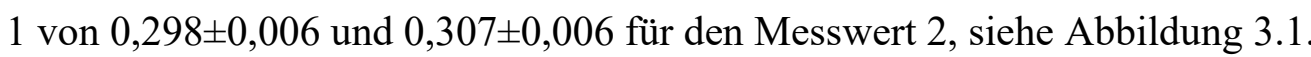

Die Signifikanztestung ergab keinen signifikanten Unterschied zwischen den Mediastinum/Thorax-Quotienten Messwerte 1 und 2 ( $\mathrm{p}=0,2)$. Die weitere Auswertung der im Boxplot dargestellten Werte kann im Anhang in Tabelle 6.1 eingesehen werden. 


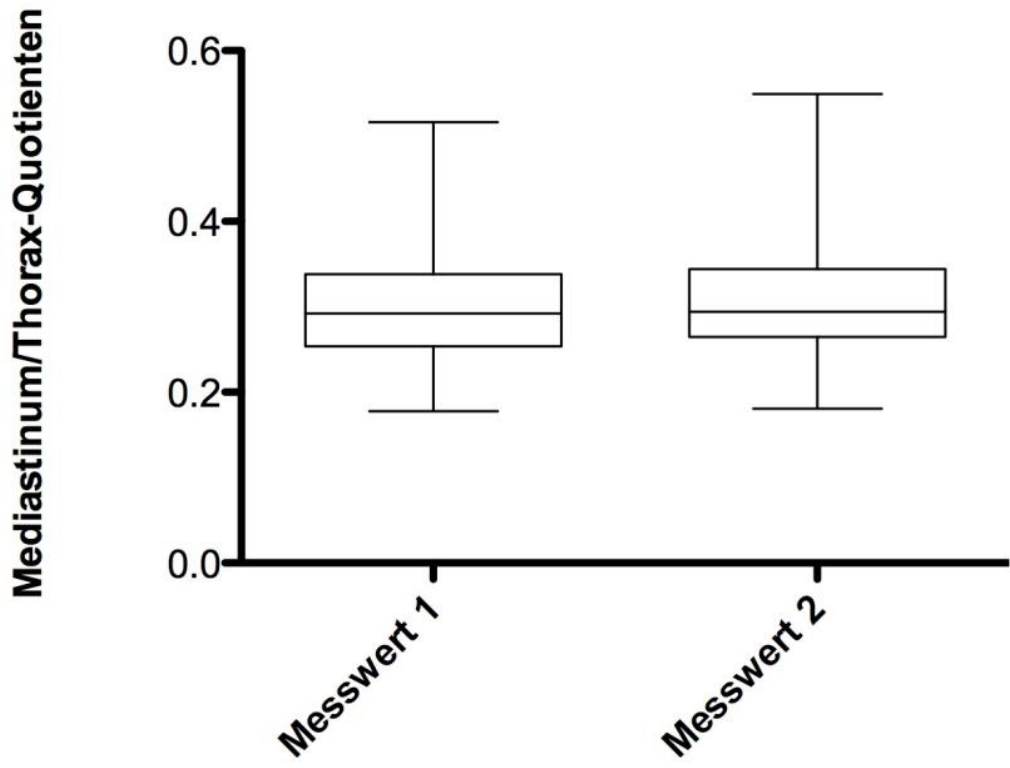

Abbildung 3.1: Boxplot-Darstellung der Mediastinum/Thorax-Quotienten-Verteilung $(n=267)$ nach Messwert 1 und 2.

In einem zweiten Schritt wurden die Mediastinum/Thorax-Quotienten (Messwert 1 und 2) anhand des Geschlechts der Patienten verglichen. Es zeigte sich ein um 0,02 abweichender Median (0,28 bis 0,3), siehe Abbildung 3.2.

Die Signifikanztestung ergab weder zwischen der Untersuchungsgruppe Männlich M1 und Weiblich M1 $(p=0,536)$ noch zwischen der Analysegruppe Männlich M2 und Weiblich M2 ( $\mathrm{p}=0,323)$ einen signifikanten Unterschied.

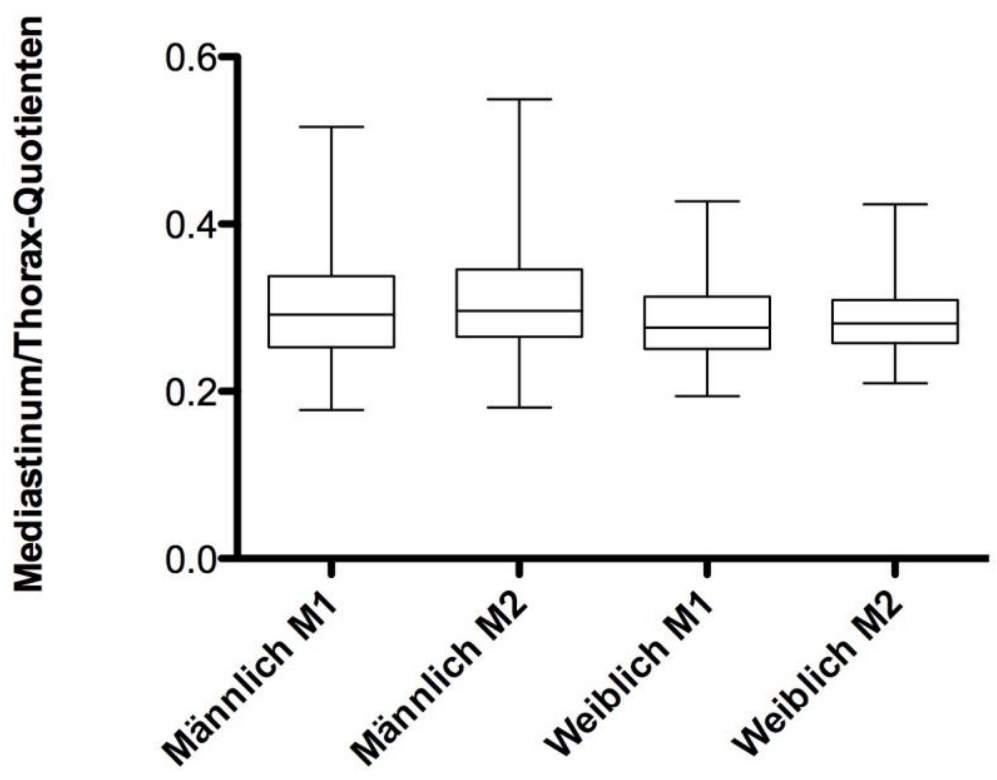

Abbildung 3.2: Boxplot-Darstellung des Mediastinum/Thorax-Quotienten nach Messwert Männlich M1 und M2 sowie Messwert Weiblich M1 und M2 (männlich n=205, weiblich n=62). 


\subsubsection{Mediastinum-Quotienten der Untersuchungsgruppen}

Die Untersuchungsgruppe Auffällig setzte sich aus 27 Patienten (85\% männlichen und $15 \%$ weiblichen) mit einem Altersdurchschnitt von $49 \pm 19$ Jahren zusammen. Es wurden 13 Aortenverletzungen und 14 Perikardtamponaden als Mediastinalverletzungen zusammengefasst. Die zweite Untersuchungsgruppe Unauffällig bestand aus 240 Patienten (77\% männlichen und 23\% weiblichen) mit einem Altersdurchschnitt von $49 \pm 21$ Jahren.

Die Untersuchungsgruppen Auffällig und Unauffällig wurden, wie in Abbildung 3.3 dargestellt, anhand der Mediastinum/Thorax-Quotienten Messwert 1 und 2 einander gegenübergestellt. Die einzelnen Durchschnittswerte der aufgeführten Untersuchungsgruppen Auffällig M1, Unauffällig M1, Auffällig M2 und Unauffällig M2 weichen jeweils nur geringfügig von 0,3 ab. Die Maximalwerte der Mediastinum/ThoraxQuotienten lagen bei der Untersuchungsgruppe Unauffällig bei 0,52 (M1) und 0,55 (M2). Bei der Vergleichsgruppe Auffällig zeigten sich Maximalwerte von 0,43 (M1) und 0,47 (M2). Die genauen Zahlenwerte der Boxplot Daten können im Anhang in Tabelle 6.1 eingesehen werden.

Die Signifikanztestung zeigte weder bei der Analyse der Untersuchungsgruppe Auffällig M1 - Unauffällig M1 ( $\mathrm{p}=0,2426)$ noch bei der Untersuchungsgruppe Auffällig M2 Unauffällig M2 ( $\mathrm{p}=0,9236)$ einen signifikanten Unterschied.

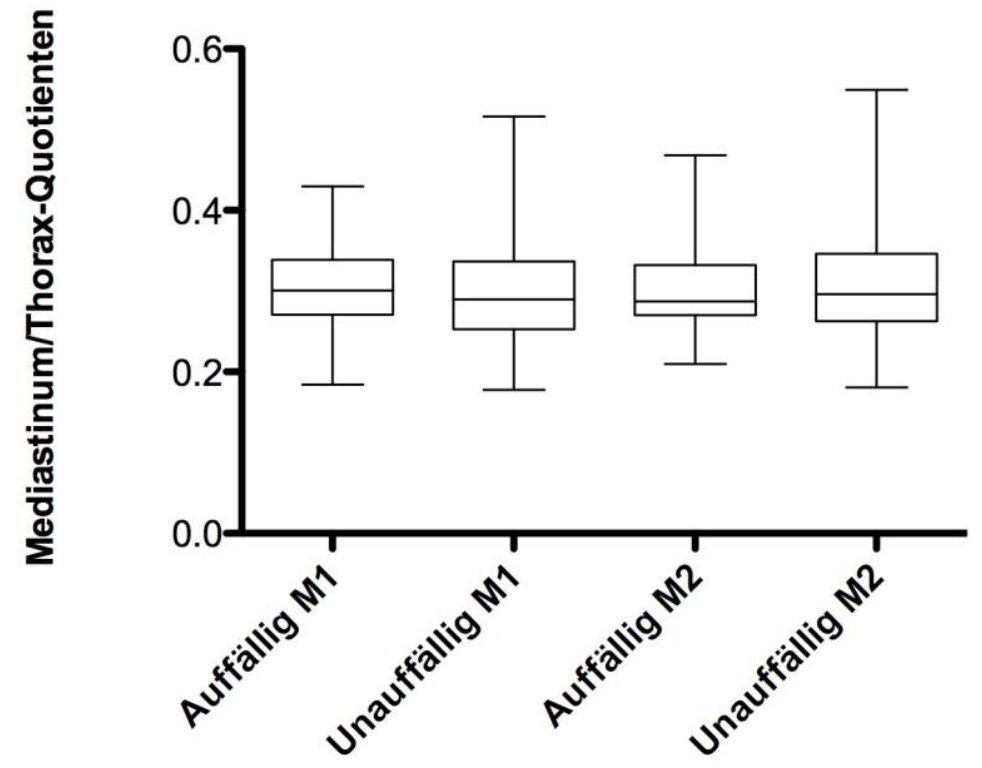

Abbildung 3.3: Boxplot-Darstellung des Mediastinum/Thorax-Quotienten nach den Untersuchungsgruppen Auffällig Messwert 1 und 2 sowie Unauffällig Messwerten 1 und 2 (Auffällig $\mathrm{n}=27$, Unauffällig $\mathrm{n}=240$ ). 


\subsubsection{Evaluation pathologischer Grenzwerte}

Die ermittelten Mediastinum/Thorax-Quotienten Messwert 1 und 2 aller 267 Patienten wurden entsprechend den definierten Grenzwerten $(\geq 0,2 / \geq 0,25 / \geq 0,28 / \geq 0,3)$ eingeteilt.

\subsubsection{Messwert 1}

Für den Mediastinum/Thorax-Quotienten Messwert 1 kann die errechnete grenzwertbezogene prozentuale Testgenauigkeit in Tabelle 3.1 eingesehen werden.

Bei einem als pathologisch definierten Grenzwert von $\geq 0,2$ waren 97\% der Messwerte 1 verbreitert. Hieraus konnte eine Sensitivität von 92,6\% und eine Spezifität von 2,9\% errechnet werden.

Bei einem Grenzwert von $\geq 0,3$ waren 39\% der Messwerte 1 verbreitert, und es konnte eine Sensitivität von $70,4 \%$ sowie eine Spezifität von $57,1 \%$ errechnet werden.

Die genaue Aufteilung der einzelnen Mediastinum/Thorax-Quotientenbereiche anhand der richtig bzw. falsch positiven/negativen Befunde können im Anhang in Tabelle 6.2 eingesehen werden.

Tabelle 3.1: Definierte Grenzwerte des Mediastinum/Thorax-Quotienten Messwert 1 ( $n=267)$ und Darstellung der grenzwert-bezogenen prozentualen Testgenauigkeit.

\begin{tabular}{lllll}
\hline Grenzwerte: & $\geq 0,2$ & $\geq 0,25$ & $\geq 0,28$ & $\geq 0,30$ \\
\hline Sensitivität & $92,6 \%$ & $81,5 \%$ & $70,4 \%$ & $70,4 \%$ \\
Spezifität & $2,9 \%$ & $22,5 \%$ & $43,3 \%$ & $57,1 \%$ \\
Pos. präd. Wert & $9,7 \%$ & $10,6 \%$ & $12,3 \%$ & $15,6 \%$ \\
Neg. präd. Wert & $77,8 \%$ & $91,5 \%$ & $92,9 \%$ & $94,5 \%$ \\
\hline
\end{tabular}

\subsubsection{Messwert 2}

Für den Mediastinum/Thorax-Quotienten Messwert 2 kann die errechnete grenzwertbezogene-prozentuale Testgenauigkeit in Tabelle 3.2 eingesehen werden.

Bei einem als pathologisch definierten Grenzwert von $\geq 0,2$ waren 99,6\% der Messwerte 2 verbreitert. Es konnte eine Sensitivität von 100\% und eine Spezifität von 0,4\% berechnet werden. 
Bei einem Grenzwert von $\geq 0,28$ waren $62 \%$ der Messwerte 2 verbreitert und es konnte eine Sensitivität von 63\% sowie eine Spezifität von 38\% errechnet werden.

Die genaue Aufteilung der einzelnen Mediastinum/Thorax-Quotientenbereiche anhand der richtig bzw. falsch positiven/negativen Befunde kann der Tabelle 6.3 im Anhang entnommen werden.

Tabelle 3.2: Definierte Grenzwerte des Mediastinum/Thorax-Quotienten Messwert 2 ( $\mathrm{n}=267)$ und Darstellung der grenzwert-bezogenen prozentualen Testgenauigkeit.

\begin{tabular}{lllll}
\hline Grenzwerte: & $\geq 0,2$ & $\geq 0,25$ & $\geq 0,28$ & $\geq 0,30$ \\
\hline Sensitivität & $100 \%$ & $85,2 \%$ & $63,0 \%$ & $44,4 \%$ \\
Spezifität & $0,4 \%$ & $16,7 \%$ & $38,3 \%$ & $52,9 \%$ \\
Pos. präd. Wert & $10,2 \%$ & $10,3 \%$ & $10,3 \%$ & $9,6 \%$ \\
Neg. präd. Wert & $100 \%$ & $90,9 \%$ & $90,2 \%$ & $89,4 \%$ \\
\hline
\end{tabular}

\subsection{Früherkennung mediastinaler Verletzungen}

Die Untersuchungsgruppen Auffällig und Unauffällig wurden hinsichtlich patientenbezogener Daten, Unfallmechanismus, unfallort- und schockraum-bezogener Daten (Vitalparameter und invasive Maßnahmen), Trauma-Scores und der Letalität verglichen.

\subsubsection{Patienten-bezogene Daten und Herz/Thorax-Quotient}

Das gesamte Patientenkollektiv wurde in die Untersuchungsgruppen Auffällig und Unauffällig unterteilt und hinsichtlich des Geschlechtes einander gegenübergestellt. Die Signifikanztestung ergab keinen signifikanten Unterschied $(p=0,27523)$. Weitere deskriptive Untersuchungsparameter können der im Anhang aufgeführten Tabelle 6.1 entnommen werden.

Beim Vergleich des Herz/Thorax-Quotienten ergab sich eine durchschnittliche Herzsilhouettenbreite des gesamten Patientenkollektivs von 136,5 $\pm 23,8 \mathrm{~mm}$, bei der Untersuchungsgruppe Auffällig von 139,5 $\pm 26,2 \mathrm{~mm}$ und 136,2 $\pm 23,5 \mathrm{~mm}$ bei der Vergleichsgruppe Unauffällig.

Bei der Untersuchungsgruppe Auffällig hatten sechs Patienten (22\%) einen verbreiterten und 21 Patienten (88\%) einen normalen Herz/Thorax-Quotient, siehe Abbildung 3.4. 
Bei der Versuchsgruppe Unauffällig zeigte sich bei 62 Patienten (26\%) ein vergrößerter und bei 178 Patienten (84\%) ein normaler Herz/Thorax-Quotient.

Die Signifikanztestung ergab einen nicht signifikanten Unterschied $(p=0,44256)$. Weitere Analysedaten können im Anhang der Tabelle 6.4 entnommen werden.

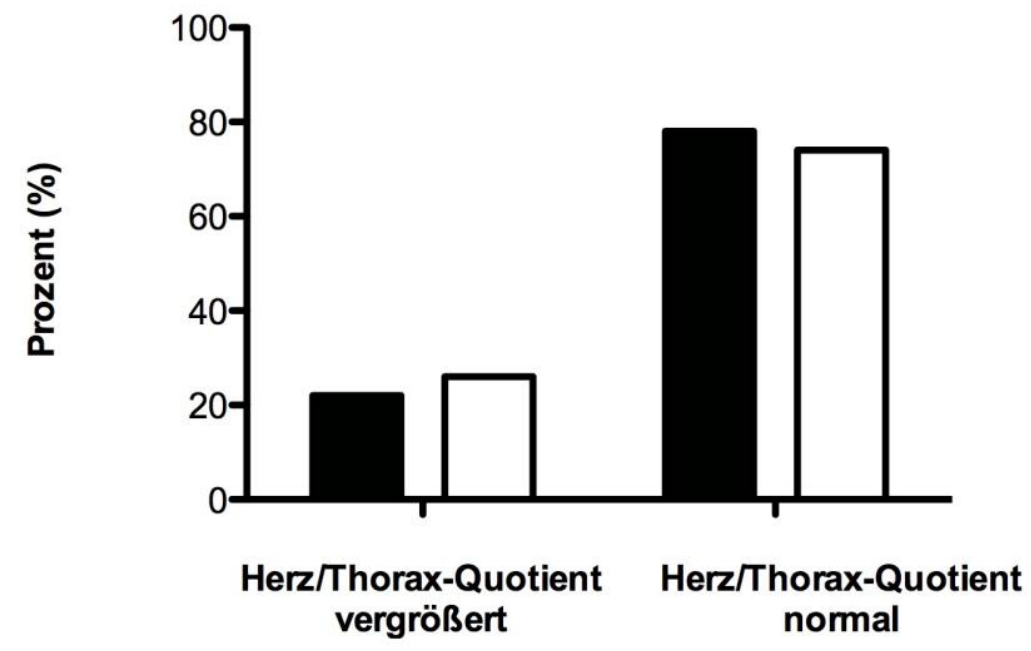

Abbildung 3.4: Balkendiagramm zur Darstellung der prozentualen Personenverteilung der Untersuchungsgruppen Auffällig (schwarzer Balken: $\mathrm{n}_{\text {vergrößert }}=6,22 \% ; \mathrm{n}_{\text {normal }}=21,78 \%$ ) und Unauffällig (weißer Balken: $\mathrm{n}_{\text {vergrößert }}=62,26 \% ; \mathrm{n}_{\text {normal }}=178,74 \%$ ) nach definitionsgemäß vergrößertem und normalem Herz/Thorax-Quotient.

\subsubsection{Thoraxverletzungsstatus}

Die Untersuchungsgruppen wurden hinsichtlich der in vier Schweregrade (0 keine, I leicht, II mittelschwer, III schwer) eingeteilten Thoraxverletzungen miteinander verglichen. Es zeigte sich, dass mit zunehmender Verletzungsschwere des Thorax die Anzahl der Patienten aus Gruppe Unauffällig nahezu kontinuierlich abnahm (Ausnahme mittelschwere Thoraxverletzung). Die meisten Patienten der Untersuchungsgruppe Unauffällig befanden sich in der Kategorie keine und leichte Thoraxverletzungen, siehe Abbildung 3.5.

Bei der Vergleichsgruppe Auffällig zeigte sich, dass mit zunehmender Verletzungsschwere des Thorax die Patientenzahl anstieg. Von den elf dokumentierten Thoraxverletzungen der Untersuchungsgruppe Auffällig waren drei Patienten (27\%) bei den mittelschweren- und acht Patienten (73\%) bei den schweren Thoraxverletzungen zu finden. 
Bei der Signifikanztestung zeigte sich ein signifikant unterschiedlicher $p$-Wert $(\mathrm{p}=0,000005)$. Genauere Angaben zur Patientenverteilung können im Anhang in Tabelle 6.4 eingesehen werden.

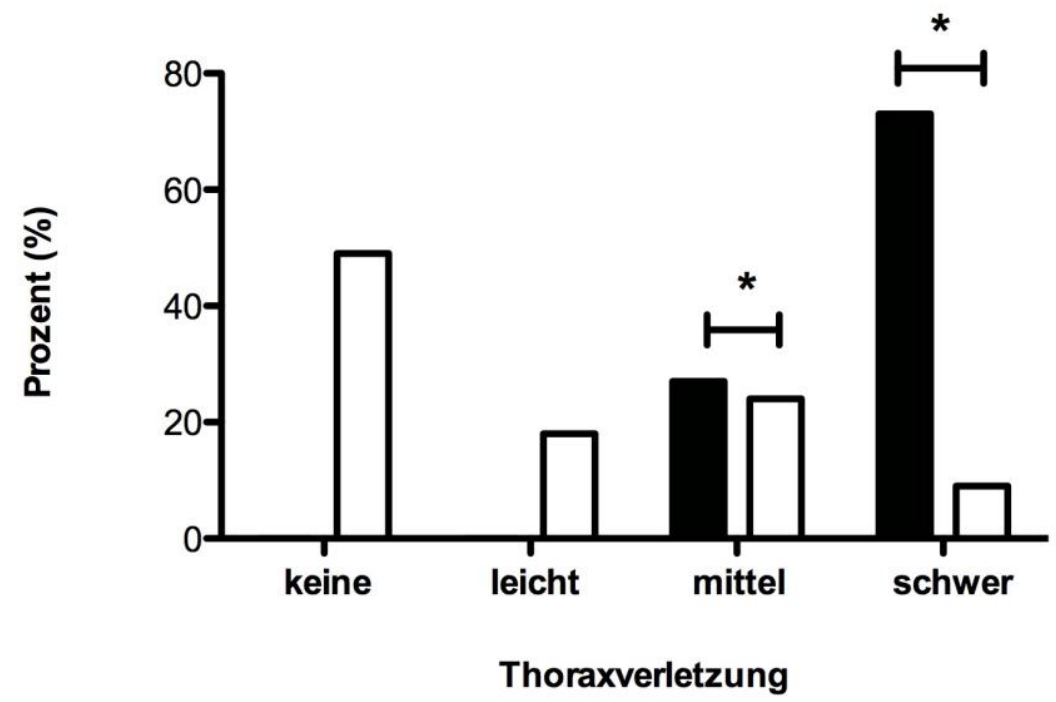

Abbildung 3.5: Balkendiagramm zur Darstellung der prozentualen Personenverteilung der Untersuchungsgruppen Auffällig (schwarzer Balken: $\mathrm{n}_{\text {mittel }}=3,27 \% ; \mathrm{n}_{\text {schwer }}=8,73 \%$ ) und Unauffällig (weißer Balken: $\mathrm{n}_{\text {keine }}=95,49 \% ; \mathrm{n}_{\text {leicht }}=35,18 \% ; \mathrm{n}_{\text {mittel }}=46,24 \% ; \mathrm{n}_{\text {schwer }}=18,9 \%$ ) nach der Verletzungsschwere des Thorax. Signifikanter Unterschied $(\mathrm{p} \leq 0,002381)$ mittels * gekennzeichnet.

\subsubsection{Verletzungsursachen}

Die Untersuchungsgruppen wurden hinsichtlich der häufigsten Verletzungsursachen (Verkehrsunfall, Sturz, sonstige: Stich-, Schlag-, Treppensturz-, Explosions-, Brand-, unklare Verletzung) miteinander verglichen. Es zeigte sich, dass insgesamt 135 Patienten (10 aus Gruppe Auffällig und 125 aus Gruppe Unauffällig) einen Verkehrsunfall erlitten haben, siehe Abbildung 3.6.

Die Signifikanztestung ergab einen nicht signifikanten $p$-Wert $(p=0,154)$. Die Darstellung der Patientenanzahl der einzelnen Verletzungsursachen kann in Tabelle 6.5 im Anhang eingesehen werden. 


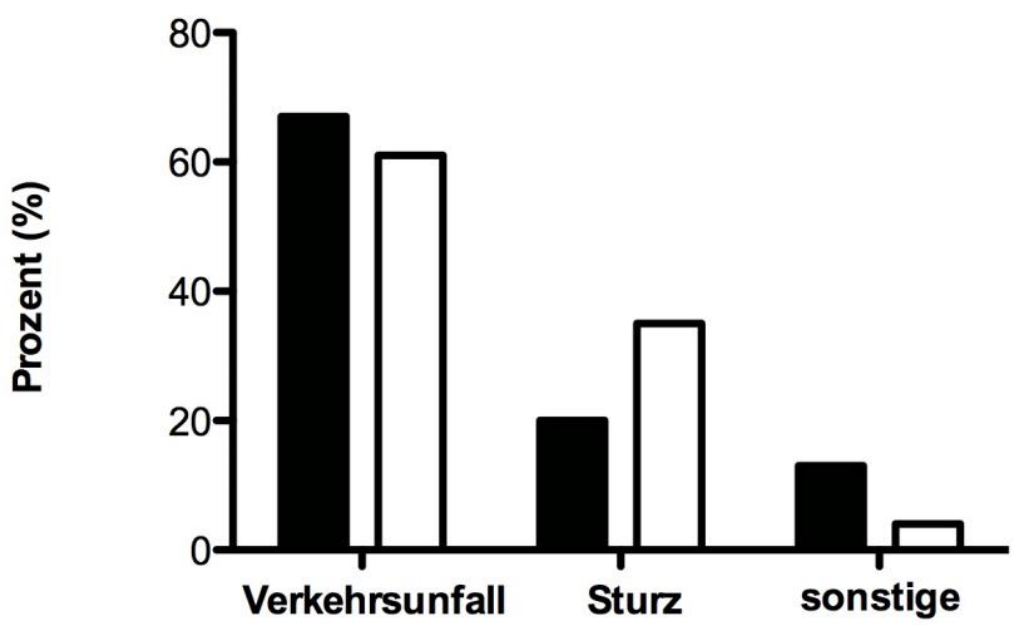

Abbildung 3.6: Balkendiagramm zur Darstellung der prozentualen Personenverteilung der Untersuchungsgruppen Auffällig (schwarzer Balken: $\mathrm{n}_{\text {Verkehrsunfall }}=10,67 \% ; \mathrm{n}_{\text {Sturz }}=3,20 \%$; $\mathrm{n}_{\text {sonstige }}=2,13 \%$ ) und Unauffällig (weißer Balken: $\mathrm{n}_{\text {Verkehrsunfall }}=125,61 \% ; \mathrm{n}_{\text {Sturz }}=72,35 \%$; $\mathrm{n}_{\text {sonstige }}=8,4 \%$ ) nach den dokumentierten Ursachen der Thoraxverletzung.

\subsubsection{Vitalparameter Unfallort und Schockraum}

Die Analyse von Blutdruck (RR), Herzfrequenz (HF), Sauerstoffsättigung $\left(\mathrm{SpO}_{2}\right)$, Intubations-, Thoraxdrainage- und Katecholaminstatus fand zu zwei unterschiedlichen Erhebungszeitpunkten statt, einmal präklinisch am Unfallort (Zeitpunkt A) und einmal klinisch im Schockraum (Zeitpunkt B).

\subsubsection{Systolischer RR}

Die Untersuchungsgruppe Auffällig (Unfallort/Schockraum) und Unauffällig (Unfallort/Schockraum) wurden, wie in Abbildung 3.7 dargestellt, hinsichtlich des auf den Schockraumbögen dokumentierten RR verglichen.

Die Analyse der Untersuchungsgruppen zeigte bei der Gruppe Auffällig einen durchschnittlichen systolischen RR am Unfallort von $133 \pm 39,4 \mathrm{mmHg}$ und im Schockraum von 122 $\pm 32,6 \mathrm{mmHg}$. Bei der Untersuchungsgruppe Unauffällig ergab sich am Unfallort ein durchschnittlicher RR von $134 \pm 25,3 \mathrm{mmHg}$ am Unfallort und $126 \pm 28,6 \mathrm{mmHg}$ im Schockraum. Das 1. Quantil der Untersuchungsgruppe Auffällig im Schockraum lag bei $100 \mathrm{mmHg}$.

Die Signifikanztestung ergab weder zum Zeitpunkt A $(p=0,7125)$ noch zu Zeitpunkt B $(p=0,598)$ einen signifikanten Unterschied der Untersuchungsgruppen. Weitere deskriptive Daten der Abbildung 3.7 können im Anhang der Tabelle 6.6 entnommen werden. 


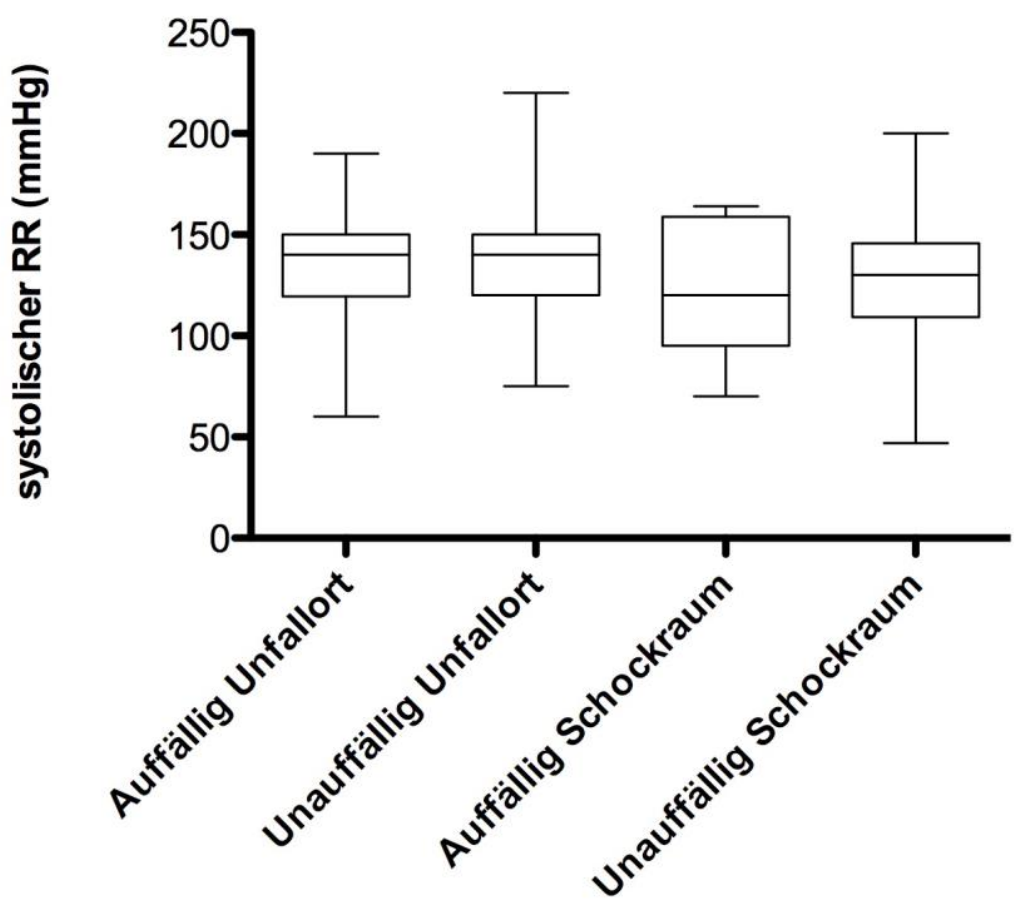

Abbildung 3.7: Boxplot-Darstellung der systolischen Blutdruckwerte in $\mathrm{mmHg}$ der Untersuchungsgruppen Auffällig ( $\mathrm{n}_{\text {Unfallort }}=12, \mathrm{n}_{\text {Schockraum }}=14$ ) und Unauffällig ( $\mathrm{n}_{\text {Unfallort }}=189$, $\mathrm{n}_{\text {Schockraum }}=196$ ) nach Zeitpunkt A (Unfallort) und Zeitpunkt B (Schockraum).

\subsubsection{HF-Werte}

Die Untersuchungsgruppen Auffällig (Unfallort/Schockraum) und Unauffällig (Unfallort/Schockraum) wurden ebenfalls, hinsichtlich der auf den Schockraumbögen dokumentierten HF-Werte verglichen, siehe Abbildung 3.8.

Bei der Analyse ergab sich für die Gruppe Auffällig eine durchschnittliche HF am Unfallort von 101 $\pm 33 / \mathrm{min}$ und im Schockraum von 90 $\pm 30 / \mathrm{min}$. Bei der Untersuchungsgruppe Unauffällig wurde am Unfallort eine durchschnittliche HF von $88 \pm 21 /$ min und $85 \pm 22 /$ min im Schockraum gemessen. Die maximal gemessene HF der Untersuchungsgruppe Unauffällig betrug am Unfallort 199/min und im Schockraum 195/min, die minimale HF am Unfallort bei 16/min bzw. 18/min im Schockraum.

Die durchgeführte Signifikanztestung zeigte weder zum Zeitpunkt A $(p=0,0376)$ noch zum Zeitpunkt B $(\mathrm{p}=0,5)$ einen signifikanten Unterschied. Weitere deskriptive Analysedaten der Abbildung 3.8 können im Anhang der Tabelle 6.6 entnommen werden. 


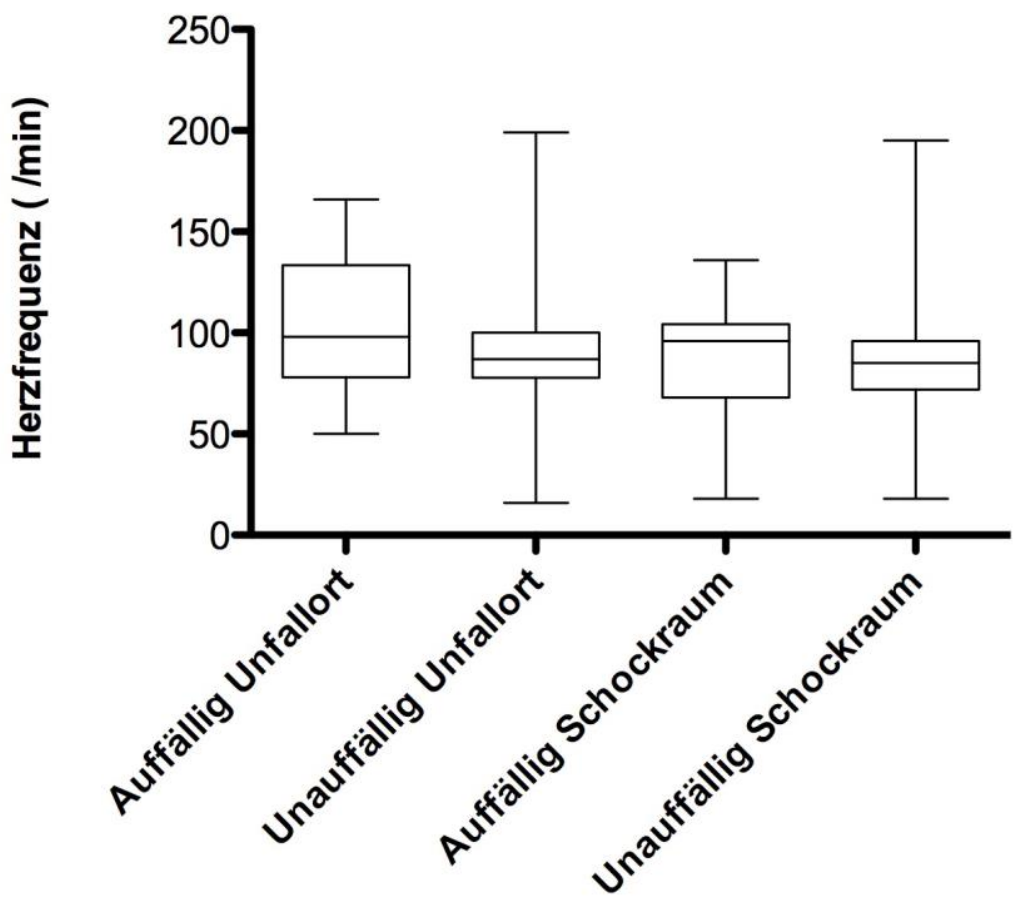

Abbildung 3.8: Boxplot-Darstellung der dokumentierten Herzfrequenz pro Minute der Untersuchungsgruppen Auffällig ( $\mathrm{n}_{\text {Unfallort }}=13, \mathrm{n}_{\text {Schockraum }}=14$ ) und Unauffällig ( $\mathrm{n}_{\text {Unfallort }}=186$, $\mathrm{n}_{\text {Schockraum }}=198$ ) nach Zeitpunkt A (Unfallort) und Zeitpunkt B (Schockraum).

\subsubsection{3 $\mathrm{SpO}_{2}$-Sättigung}

Im Folgenden wurden die Untersuchungsgruppen Auffällig und Unauffällig hinsichtlich der auf den Schockraumbögen dokumentierten $\mathrm{SpO}_{2}$-Sättigung verglichen.

Aufgeteilt in die Untersuchungsgruppen ergab sich bei der Gruppe Auffällig eine durchschnittliche $\mathrm{SpO}_{2}$-Sättigung von $88 \pm 11 \%$ am Unfallort und $95 \pm 5 \%$ im Schockraum. Bei der Untersuchungsgruppe Unauffällig zeigte sich am Unfallort eine durchschnittliche $\mathrm{SpO}_{2}$-Sättigung von $93 \pm 9 \%$ und $96 \pm 6 \%$ im Schockraum.

Die Signifikanztestung ergab weder zum Zeitpunkt A $(p=0,0316)$ noch zum Zeitpunkt B $(p=0,2834)$ einen signifikanten Unterschied. Weitere deskriptive Daten sowie die genaue Zusammensetzung der Untersuchungsgruppen können im Anhang in Tabelle 6.6 eingesehen werden.

\subsubsection{Maßnahmen und Medikamente}

\subsubsection{Intubation}

Es wurden die Untersuchungsgruppen Auffällig und Unauffällig hinsichtlich des dokumentierten Intubationsstatus miteinander verglichen, siehe Abbildung 3.9. Hierbei 
zeigte sich, dass die Zahl der Intubationen bei der Patientengruppe Auffällig mit 47\% stattgefundenen Intubationen $\mathrm{zu}$ den Zeitpunkten A und B identisch waren. Bei der Patientengruppe Unauffällig wurde bei 38\% der Patienten am Unfallort und bei 11\% der Patienten im Schockraum eine Intubation durchgeführt.

Die Signifikanztestung zeigte zum Zeitpunkt A (Unfallort) einen nicht signifikanten $(\mathrm{p}=0,18991)$ und zum Zeitpunkt B (Schockraum) einen signifikanten Unterschied ( $\mathrm{p}=0,00135)$ zwischen den Untersuchungsgruppen Auffällig und Unauffällig.

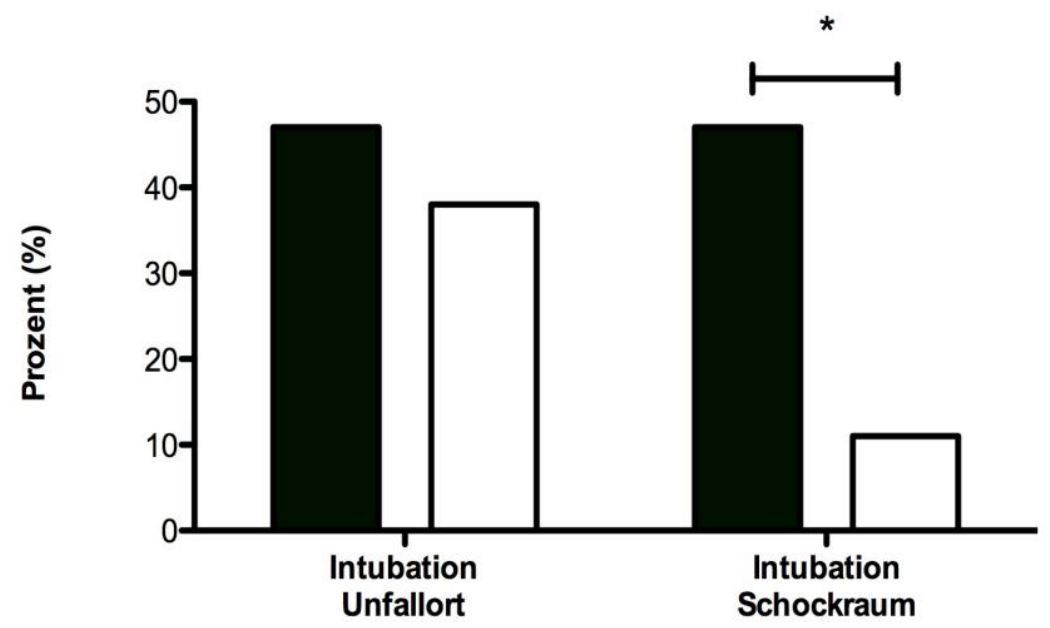

Abbildung 3.9: Balkendiagramm zur Darstellung der prozentualen Verteilung der Untersuchungsgruppen Auffällig (schwarzer Balken: $\mathrm{n}_{\text {Intubation-Unfallort }}=7,47 \% ; \quad \mathrm{n}_{\text {Intubation- }}$ Schockraum $=7,47 \%$ ) und Unauffällig (weißer Balken: $\mathrm{n}_{\text {Intubation-Unfallort }}=76,38 \%$; $\mathrm{n}_{\text {Intubation- }}$ Schockraum $=22,11 \%$ ) nach am Unfallort und im Schockraum durchgeführten Intubationen. Kennzeichnung des signifikanten Unterschiedes $(\mathrm{p} \leq 0,002381)$ mittels *.

\subsubsection{Thoraxdrainage}

Als zweite invasive Maßnahme wurden die Untersuchungsgruppen Auffällig und Unauffällig hinsichtlich des dokumentierten Thoraxdrainagestatus miteinander verglichen, siehe Abbildung 3.10. Bei der Patientengruppe Auffällig waren bei 20\% Thoraxdrainagen am Unfallort (Zeitpunkt A) und 47\% im Schockraum (Zeitpunkt B) gelegt worden. Bei der Vergleichsgruppe Unauffällig wurden 4\% der Thoraxdrainagen am Unfallort und 7\% im Schockraum gelegt.

Die Signifikanztestung ergab zum Zeitpunkt A einen nicht signifikanten (pWert=0,02696) und zum Zeitpunkt B einen signifikanten Unterschied (pWert $=0,00015$ ) der Analysegruppen. 


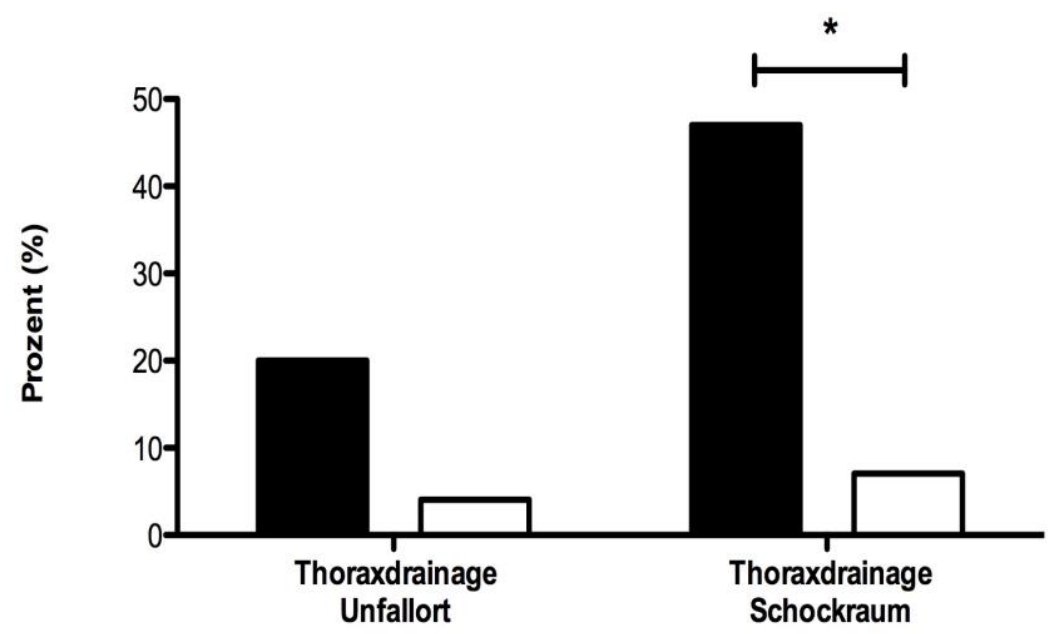

Abbildung 3.10: Balkendiagramm zur Darstellung der prozentualen Verteilung der Untersuchungsgruppen Auffällig (schwarzer Balken: $\mathrm{n}_{\text {Thoraxdrainage-Unfallort }}=3,20 \% ; \mathrm{n}_{\text {Thoraxdrainage- }}$ Schockraum $=7,47 \%$ ) und Unauffällig (weißer Balken: $\mathrm{n}_{\text {Thoraxdrainage-Unfallort }}=7,4 \% ; \mathrm{n}_{\text {Thoraxdrainage- }}$ Schockraum $=14,7 \%$ ) nach durchgeführten Thoraxdrainagen am Unfallort und im Schockraum. Kennzeichnung des signifikanten Unterschiedes $(\mathrm{p} \leq 0,002381)$ mittels *.

\subsubsection{Katecholamine}

Die Untersuchungsgruppen Auffällig und Unauffällig wurden hinsichtlich der dokumentierten Katecholamin-Gabe verglichen, siehe Abbildung 3.11. Es zeigte sich, dass $40 \%$ der Patienten aus Untersuchungsgruppe Auffällig sowohl am Unfallort als auch im Schockraum Katecholamine erhalten haben. Bei der Patientengruppe Unauffällig wurden 9\% der Patienten am Unfallort und 11\% im Schockraum Katecholamine verabreicht.

Die Signifikanztestung zeigte am Unfallort einen signifikanten $(\mathrm{p}-\mathrm{Wert}=0,002380)$ und im Schockraum einen nicht signifikanten Unterschied ( $p$-Wert=0,00569). 


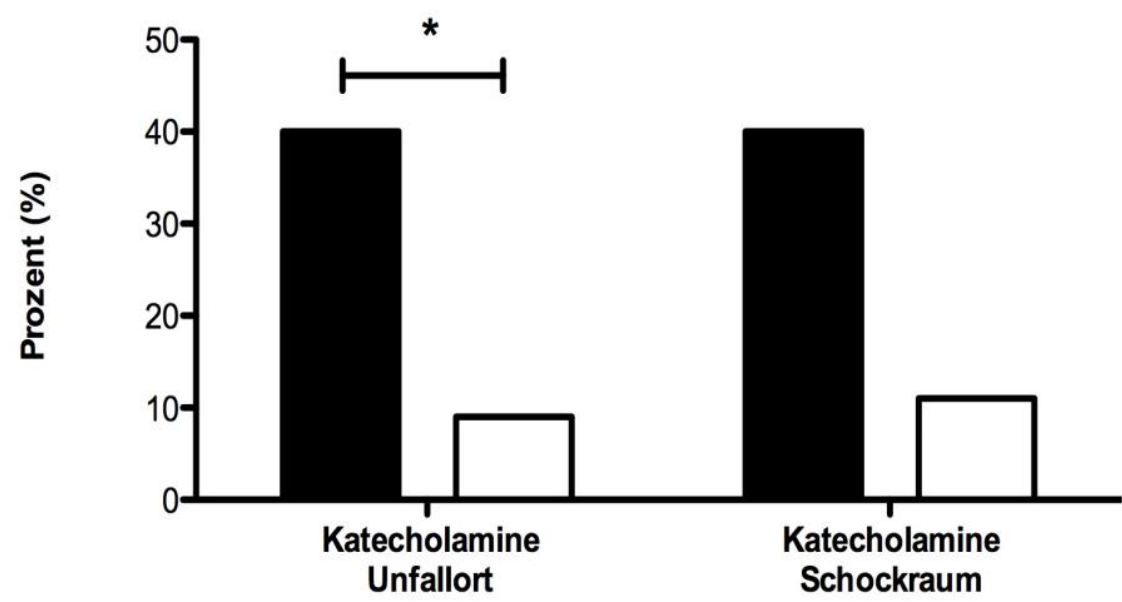

Abbildung 3.11: Balkendiagramm zur Darstellung der prozentualen Verteilung der Untersuchungsgruppen Auffällig (schwarzer Balken: $\mathrm{n}_{\text {Katecholamine-Unfallort }}=6,40 \%$; $\mathrm{n}_{\text {Katecholamine- }}$ Schockraum $=6,40 \%$ ) und Unauffällig (weißer Balken: $\mathrm{n}_{\text {Katecholamine-Unfallort }}=17,9 \%$; $\mathrm{n}_{\text {Katecholamine- }}$ Schockraum $=22,11 \%$ ) nach Verabreichung von Katecholaminen am Unfallort und im Schockraum. Kennzeichnung der Signifikanz ( $\leq 00,002381)$ mittels *.

\subsubsection{Trauma-Scores}

\subsubsection{NISS}

Die Untersuchungsgruppen Auffällig und Unauffällig wurden hinsichtlich des errechneten NISS verglichen, siehe Abbildung 3.12. Aufgeteilt in die einzelnen Untersuchungsgruppen ergab sich für die Untersuchungsgruppe Auffällig ein NISSDurchschnittswert von $39 \pm 18$ Punkten und für die Untersuchungsgruppe Unauffällig von $28 \pm 15$.

Die Verteilung der NISS-Punktwerte reichte bei der Untersuchungsgruppe Auffällig von 15 bis 75 Punkten. Die Vergleichsgruppe Unauffällig zeigte eine Verteilung von 3 bis 75 Punkten. Das 3. Quantil lag bei der Untersuchungsgruppe Auffällig bei einem Punktwert von 50 und bei der Vergleichsgruppe Unauffällig bei 34 Punkten.

Die Signifikanztestung ergab einen nicht signifikanten Unterschied $(p=0,01663)$. Weitere deskriptive Analysedaten können der Tabelle 6.7 im Anhang entnommen werden. 


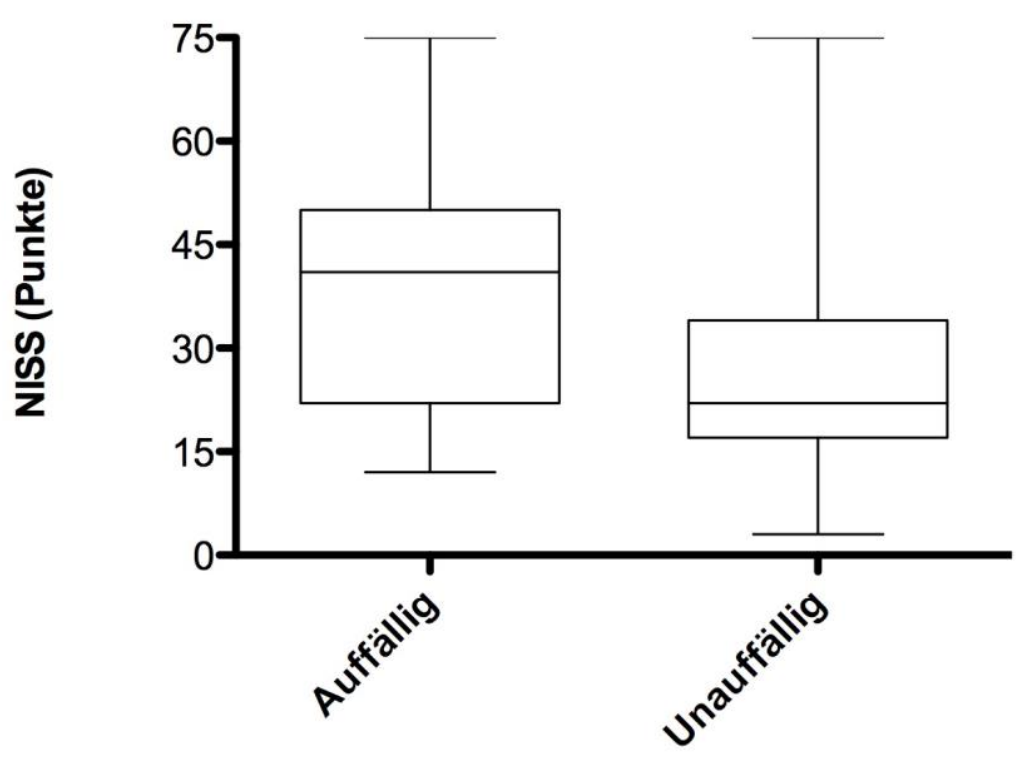

Abbildung 3.12: Boxplot-Darstellung der NISS-Punktwert-Verteilung nach den Untersuchungsgruppen Auffällig ( $\mathrm{n}=15)$ und Unauffällig ( $\mathrm{n}=206)$.

\subsubsection{RISC-II-Score}

Die Untersuchungsgruppen wurden hinsichtlich des RISC-II-Score verglichen, siehe Abbildung 3.13. Bei der Untersuchungsgruppe Auffällig zeigte sich eine durchschnittliche Überlebenswahrscheinlichkeit von $73 \pm 32 \%$, bei der Untersuchungsgruppe Unauffällig von $88 \pm 22 \%$. Das 1. Quantil der Patientengruppe Auffällig lag bei $35 \%$ und das der Vergleichsgruppe Unauffällig bei 91\%.

Die Signifikanztestung zeigte einen nicht signifikanten Unterschied $(p=0,01517)$. Weitere Analysedaten können der Tabelle 6.7 im Anhang entnommen werden.

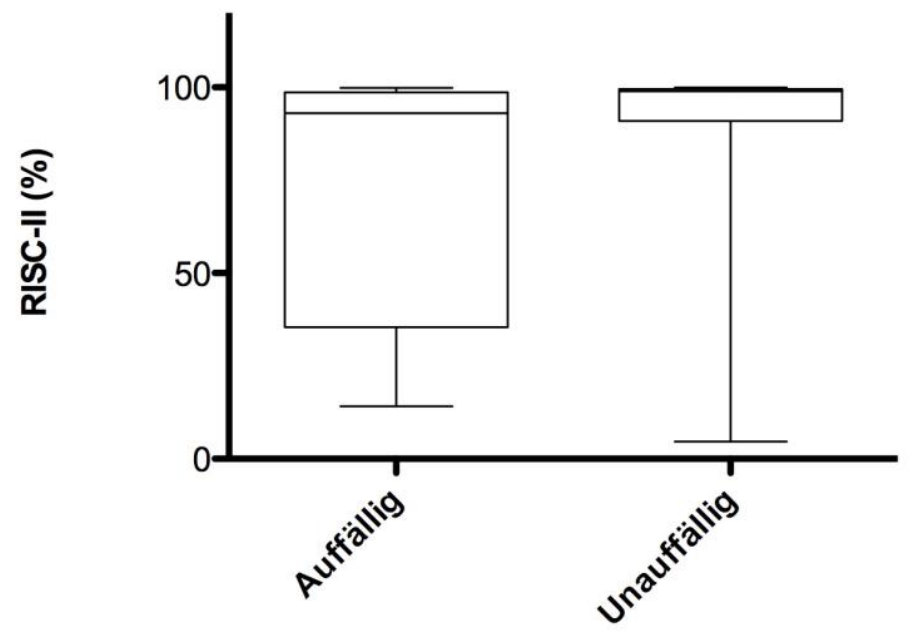

Abbildung 3.13: Boxplot-Darstellung der prozentualen Verteilung des RISC-II-Score nach den Untersuchungsgruppen Auffällig ( $\mathrm{n}=15)$ und Unauffällig ( $\mathrm{n}=194)$. 


\subsubsection{Letalität}

Es wurden die Untersuchungsgruppen Auffällig und Unauffällig hinsichtlich der Letalität verglichen, siehe Abbildung 3.14. Es zeigte sich bei der Untersuchungsgruppe Auffällig eine Letalität von $15 \%$ und bei der Vergleichsgruppe Unauffällig von 7,5\%.

Die Signifikanztestung ergab einen nicht signifikanten Unterschied $(p=0,203)$. Die genauen Patientendaten können im Anhang in Tabelle 6.8 eingesehen werden.

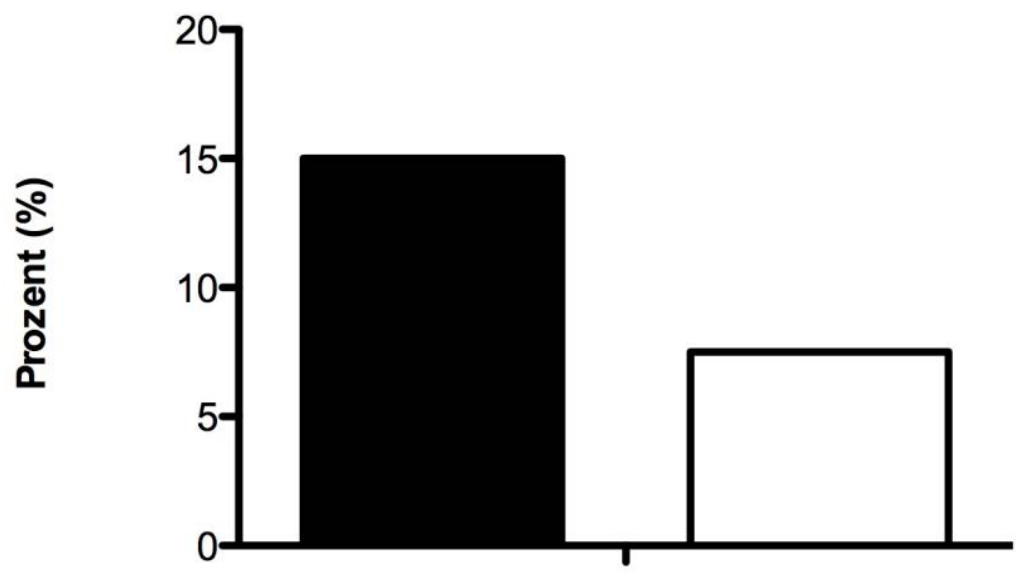

Verstorben

Abbildung 3.14: Balkendiagramm zur Darstellung der prozentualen Letalität nach den Untersuchungsgruppen Auffällig (schwarzer Balken: $\mathrm{n}_{\text {verstorben }}=4,15 \%$ ) und Unauffällig (weißer Balken: $\mathrm{n}_{\text {verstorben }}=18,7,5 \%$ ).

\subsection{8 Übersicht Signifikanztestung}

Tabelle 3.3: Darstellung analysierter Variablen, angewandter Signifikanztests sowie angepassten Signifikanzniveaus und p-Werts.

\begin{tabular}{llll}
\hline Variablen & Signifikanztest & Signifikanzniveau & $\mathrm{p}$-Wert \\
\hline $\begin{array}{l}\text { Verhältnis Med./Thor.-Quotient } \\
\text { Messwert 1 - Messwert 2 }\end{array}$ & Mann-Whitney-U-Test & $\mathrm{p} \leq 0,017$ & 0,2 \\
$\begin{array}{l}\text { Geschlechterverhältnis } \\
\text { Med./Thor.-Quotient M1 }\end{array}$ & Mann-Whitney-U-Test & $\mathrm{p} \leq 0,017$ & 0,536 \\
$\begin{array}{l}\text { Geschlechterverhältnis } \\
\text { Med./Thor.-Quotient M2 }\end{array}$ & Mann-Whitney-U-Test & $\mathrm{p} \leq 0,017$ & 0,323 \\
\hline
\end{tabular}


Tabelle 3.4: Darstellung analysierter Variablen, angewandter Signifikanztests sowie angepasstem Signifikanzniveau und p-Wert. Signifikanz mittels * gekennzeichnet.

\begin{tabular}{|c|c|c|c|}
\hline Variablen & Signifikanztest & Signifikanzniveau & $\mathrm{p}$-Wert \\
\hline Geschlechterverhältnis & Pearson-Chi-Square-Test & $\mathrm{p} \leq 0,002381$ & 0,27523 \\
\hline Med./Thor.-Quotient 1 & Mann-Whitney-U-Test & $\mathrm{p} \leq 0,002381$ & 0,2426 \\
\hline Med./Thor.-Quotient 2 & Mann-Whitney-U-Test & $\mathrm{p} \leq 0,002381$ & 0,9236 \\
\hline Herz/Thor.-Verhältnis & Fisher-Exact-Test & $\mathrm{p} \leq 0,002381$ & 0,44256 \\
\hline Thoraxverletzung & Mann-Whitney-U-Test & $\mathrm{p} \leq 0,002381$ & $0,000005 *$ \\
\hline Verletzungsursache & Fisher-Exact-Test & $\mathrm{p} \leq 0,002381$ & 0,154 \\
\hline RR Unfallort & Mann-Whitney-U-Test & $\mathrm{p} \leq 0,002381$ & 0,7125 \\
\hline RR Schockraum & Mann-Whitney-U-Test & $\mathrm{p} \leq 0,002381$ & 0,598 \\
\hline HF Unfallort & t-Test & $\mathrm{p} \leq 0,002381$ & 0,0376 \\
\hline HF Schockraum & t-Test & $\mathrm{p} \leq 0,002381$ & 0,50 \\
\hline $\mathrm{SpO}_{2}$ Unfallort & Mann-Whitney-U-Test & $\mathrm{p} \leq 0,002381$ & 0,0316 \\
\hline $\mathrm{SpO}_{2}$ Schockraum & Mann-Whitney-U-Test & $\mathrm{p} \leq 0,002381$ & 0,2834 \\
\hline Intubation Unfallort & Fisher-Exact-Test & $\mathrm{p} \leq 0,002381$ & 0,18991 \\
\hline Intubation Schockraum & Fisher-Exact-Test & $\mathrm{p} \leq 0,002381$ & $0,00135 *$ \\
\hline Thoraxdrainage Unfallort & Fisher-Exact-Test & $\mathrm{p} \leq 0,002381$ & 0,02696 \\
\hline Thoraxdrainage & Fisher-Exact-Test & $\mathrm{p} \leq 0,002381$ & $0,00015 *$ \\
\hline \multicolumn{4}{|l|}{ Schockraum } \\
\hline Katecholamine Unfallort & Fisher-Exact-Test & $\mathrm{p} \leq 0,002381$ & $0,002380 *$ \\
\hline Katecholamine & Fisher-Exact-Test & $\mathrm{p} \leq 0,002381$ & 0,00569 \\
\hline \multicolumn{4}{|l|}{ Schockraum } \\
\hline NISS & Mann-Whitney-U-Test & $\mathrm{p} \leq 0,002381$ & 0,01663 \\
\hline RISC-II-Score & Mann-Whitney-U-Test & $\mathrm{p} \leq 0,002381$ & 0,01517 \\
\hline Letalität & $\mathrm{t}$-Test & $\mathrm{p} \leq 0,002381$ & 0,203 \\
\hline
\end{tabular}




\subsection{Vergleich bildgebender Verfahren der initialen Pneumo- /Hämatothorax-Diagnostik im Schockraum}

Der Stellenwert der bildgebenden Verfahren Sonographie und Thoraxröntgenuntersuchung wurde hinsichtlich der initialen Pneumo-/Hämatothorax-Diagnostik im Schockraum und den daraus abgeleiteten Maßnahmen verglichen. Insgesamt wurden 141 Patienten untersucht. Davon waren 93 männliche und 48 weibliche Patienten. Unterteilt in die einzelnen Untersuchungsgruppen ergab sich für das eFAST eine Verteilung von 90 untersuchten Männern (65\%) und 48 Frauen (35\%). In der Untersuchungsgruppe Thoraxröntgenuntersuchung ergab sich eine Verteilung von 23 Männern (67\%) und elf Frauen (33\%). Insgesamt wurden 20 Pneumo-/Hämatothorax detektiert, von denen elf eine sofortige Drainage benötigten. Weitere deskriptive Daten können der Tabelle 3.5 entnommen werden.

Tabelle 3.5: Alter und Geschlecht des gesamten Patientenkollektivs $(n=141)$ nach bildgebenden Verfahren eFAST und Thoraxröntgenuntersuchung unterteilt.

\begin{tabular}{ll|ll}
\hline eFAST: & Geschlecht & $\begin{array}{l}\text { Thoraxröntgen- } \\
\text { untersuchung: }\end{array}$ & Geschlecht \\
\hline Männlich & $65 \%$ & Männlich & $67 \%$ \\
Weiblich & $35 \%$ & Weiblich & $33 \%$ \\
Ges.-Anzahl (n) & 138 & Ges.-Anzahl (n) & 34 \\
& & & \\
\hline eFAST & Alter & Thoraxröntgen- & Alter \\
\hline Durchschnitt & $48,5 \mathrm{y}$ & untersuchung: & $49 \mathrm{y}$ \\
Minimum & $0,4 \mathrm{y}$ & Durchschnitt & $18 \mathrm{y}$ \\
Maximum & $93 \mathrm{y}$ & Minimum & $89 \mathrm{y}$ \\
Standardabweichung & $\pm 21,2$ & Standardabweichung & $\pm 19,2$ \\
Ges.-Anzahl (n) & 138 & Ges.-Anzahl (n) & 34 \\
\hline
\end{tabular}




\subsubsection{Testgenauigkeit von eFAST und Thoraxröntgenuntersuchung}

Die Analysegruppe eFAST setzte sich aus insgesamt 138 Patienten mit einem Durchschnittsalter von 48,5 \pm 21 Jahren zusammen. Die Verteilung ergab 16 positive und 122 negative eFAST Befunde, die prozentual in Abbildung 3.15 dargestellt wurden. Die Validierung gegen den Goldstandard CT zeigte, dass alle 16 positiven eFAST Ergebnisse einen Pneumo- oder Hämatothorax aufwiesen. Bei den 122 negativen Ergebnissen wurden vier positive Ergebnisse als negativ detektiert. Mittels dieser Werte konnte eine Sensitivität von $80 \%$ (siehe Tabelle 3.6) als Testgenauigkeit für das eFAST zur Pneumo-/Hämatothorax-Diagnostik berechnet werden.

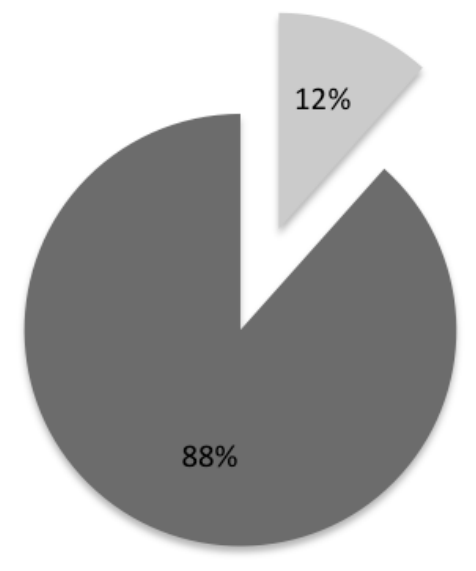

Abbildung 3.15: Prozentuale Darstellung aller analysierter eFAST Befunde unterteilt in Pneumothorax positive (hellgrau: $n=16,12 \%$ ) und negative Ergebnisse (dunkelgrau: $n=122$, $88 \%)$.

Die Analysegruppe Thoraxröntgenuntersuchung setzte sich aus insgesamt 34 Patienten mit einem Altersdurchschnitt von $49 \pm 19$ Jahren zusammen. Die Verteilung zeigte zwei positive und 32 negative Pneumo- bzw. Hämatothorax Befunde der Thoraxröntgenuntersuchung, siehe Abbildung 3.16. Die Validierung der Ergebnisse gegen den Goldstandard CT zeigte, dass beide Testergebnisse richtig positiv waren. Von den 32 negativen Ergebnissen waren neun falsch negativ eingestuft. Mittels dieser Werte konnte eine Sensitivität von 18\% (siehe Tabelle 3.6) für die Thoraxröntgenuntersuchung zur Pneumo-/Hämatothorax-Diagnostik berechnet werden. 


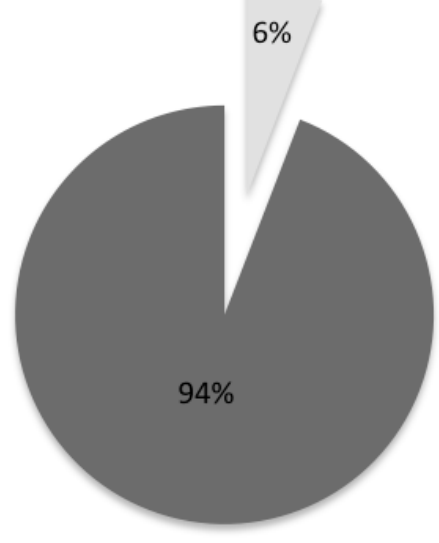

Abbildung 3.16: Prozentuale Darstellung aller Befunde der Thoraxröntgenuntersuchung unterteilt in Pneumothorax positive (hellgrau: $n=2,6 \%$ ) und negative Ergebnisse (dunkelgrau: $\mathrm{n}=32,94 \%)$.

Die nachfolgende Tabelle 3.6 zur Testgenauigkeit der Pneumo- und HämatothoraxDiagnostik zeigt für das eFAST eine Sensitivität von $80 \%$ und einen negativ prädiktiven Wert von $97 \%$. Bei der Thoraxröntgenuntersuchung konnte eine Sensitivität von $18 \%$ und ein negativ prädiktiver Wert von $71 \%$ errechnet werden.

Tabelle 3.6: Prozentuale Darstellung der Testgenauigkeit von eFAST und Thoraxröntgenuntersuchung zur initialen Pneumo-/Hämatothorax-Diagnostik im Schockraum.

\begin{tabular}{lcc} 
Untersuchung: & eFAST & Thoraxröntgenuntersuchung \\
\hline Sensitivität & $80 \%$ & $18 \%$ \\
Spezifität & $100 \%$ & $100 \%$ \\
pos. prädiktiver Wert & $100 \%$ & $100 \%$ \\
neg. prädiktiver Wert & $97 \%$ & $71 \%$
\end{tabular}

\subsubsection{Konsequenzen aus eFAST und Thoraxröntgenuntersuchung}

Die nachfolgende Tabelle 3.7 veranschaulicht die Anzahl direkt aus der initialen Schockraum-Diagnostik eingeleiteten Konsequenzen. Insgesamt wurden 141 Patienten analysiert. Weitere deskriptive Daten können der Tabelle 3.5 entnommen werden. 


\subsubsection{1 eFAST}

Das eFAST wurde bei insgesamt 138 Patienten durchgeführt. Aufgeteilt in die Untersuchungsgruppen setzte sich die Gruppe eF 1 (eFAST und CT) aus 107 Patienten und Gruppe eF 2 (eFAST, Thoraxröntgenuntersuchung und CT) aus 31 Patienten zusammen. Insgesamt wurden 64\% aller initiierten Konsequenzen direkt als Folge aus dem eFAST eingeleitet.

\subsubsection{Thoraxröntgenuntersuchung}

Die Thoraxröntgenuntersuchung wurde insgesamt bei 34 Patienten durchgeführt. Die Gruppe RöT 1 (Thoraxröntgenuntersuchung und CT) bestand aus drei Patienten, die Gruppe RöT 2 (Thoraxröntgenuntersuchung, eFAST und CT) aus 31 Patienten. Es wurde weder aus Gruppe RöT 1 noch aus Gruppe RöT 2 eine Konsequenz aufgrund der Thoraxröntgenuntersuchung eingeleitet.

\subsubsection{CT}

Das CT stellte das Validierungsmedium der von eFAST und Thoraxröntgenuntersuchung erhobenen Befunde dar. Es wurden alle 141 analysierten Patienten noch im Schockraum mittels CT untersucht. Es zeigten sich insgesamt elf entlastungswürdige Befunde, wovon sieben (64\%) durch das eFAST diagnostiziert und behandelt wurden. Vier Patienten (36\%) mit entlastungwürdigem Befund wurden weder vom eFAST noch der Thoraxröntgenuntersuchung als pathologisch erkannt.

Tabelle 3.7: Darstellung der Konsequenzen aus der initialen Schockraumbildgebung aufgeteilt nach den einzelnen Untersuchungsgruppen.

\begin{tabular}{lcccc}
\hline $\begin{array}{l}\text { Untersuchungs- } \\
\text { gruppen: }\end{array}$ & $\begin{array}{l}\text { Ges.-Anzahl } \\
\text { untersuchter } \\
\text { Patienten (n) }\end{array}$ & $\begin{array}{l}\text { Untersuchungs- } \\
\text { bedingte } \\
\text { Konsequenzen }\end{array}$ & $\begin{array}{l}\text { Ges.-Anzahl } \\
\text { durchgeführter } \\
\text { Konsequenzen }\end{array}$ & $\begin{array}{l}\text { Untersuchungs- } \\
\text { bedingte } \\
\text { Konsequenzen }\end{array}$ \\
\hline eFAST gesamt: & 138 & 7 & 11 & $64 \%$ \\
\hline - Gruppe eF 1 & 107 & 2 & 4 & $50 \%$ \\
- Gruppe eF 2 & 31 & 5 & 7 & $71 \%$ \\
\hline $\begin{array}{l}\text { Thoraxröntgen- } \\
\text { untersuchung } \\
\text { gesamt: }\end{array}$ & 34 & 0 & 0 & $0 \%$ \\
\hline - Gruppe RöT 1 & 3 & 0 & 7 & $0 \%$ \\
- Gruppe RöT 2 & 31 & 0 & 11 & $36 \%$ \\
\hline CT gesamt: & 141 & 4 & & $0 \%$ \\
\hline
\end{tabular}




\subsection{Analyse von Thoraxröntgenuntersuchung und cCT/Ganzkörper- CT verstorbener Schockraumpatienten}

Im Jahr 2013 und 2014 sind 13\% aller über den Schockraum der UMG eingelieferten Patienten verstorben. Von diesen insgesamt 184 verstorbenen Patienten waren $63 \%$ männlich und 27\% weiblich. Der Altersdurchschnitt der Patienten lag bei $75 \pm 15$ Jahren bei einer Altersverteilung von 17 bis 99 Jahren.

Es zeigte sich, dass noch vor dem Ableben bei 76\% (139 Patienten) eine CT-Diagnostik (cCT/Ganzkörper-CT) und bei 41\% (74 Patienten) eine Thoraxröntgenuntersuchung durchgeführt wurde, siehe Abbildung 3.17.

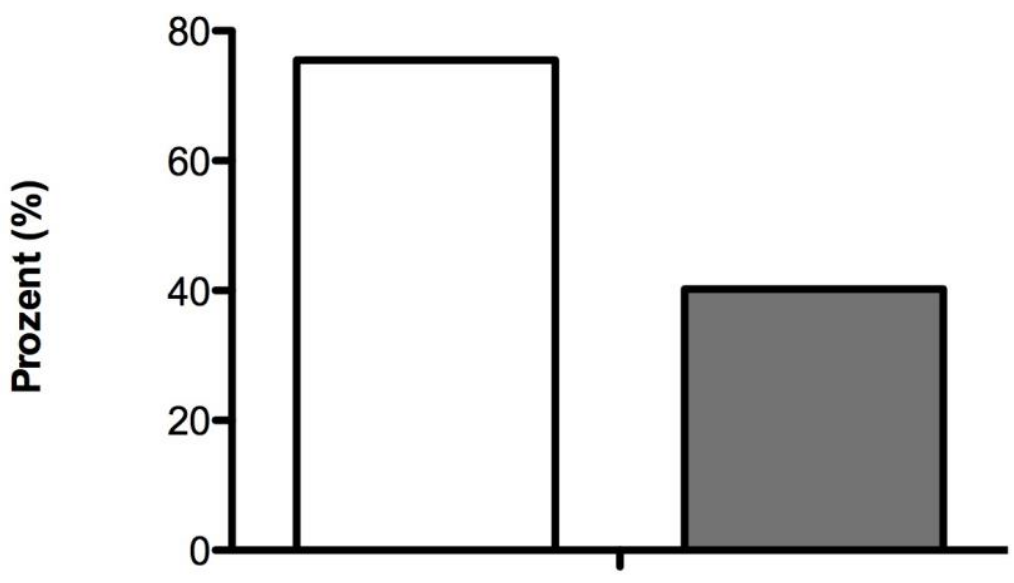

\section{Verstorben}

Abbildung 3.17: Balkendiagramm zur Darstellung der prozentualen Verteilung durchgeführter Thoraxröntgenuntersuchungen (grauer Balken: $n=74,41 \%$ ) und CT (weißer Balken: $n=139$, $76 \%$ ) nach im Schockraum der UMG verstorbenen Patienten. 


\section{Diskussion}

\subsection{Die Rolle der Thoraxröntgenuntersuchung zur Beurteilung des Mediastinums im Schockraum}

Schwere Thoraxverletzungen sind bei polytraumatisierten Patienten mit einer hohen Mortalitäts- und Morbiditätsrate verbunden (Liener et al. 2006). Rupturierte Aortenaneurysmen beispielsweise kommen in 90\% der Fälle im hämorrhagischen Schock in die Klinik und müssen schnellstmöglich erkannt und operativ versorgt werden (Johansen et al. 1991, Keßler et al. 2002). Ein mögliches Diagnosemittel thorakaler Verletzungen ist die Thoraxröntgenuntersuchung. Dabei können über mediastinale Hämatomzeichen, wie eine anormale Aortenkontur, eine Verschattung des aortopulmonalen Fensters, eine Verlagerung des Beatmungstubus und ein linksseitiger Hämatothorax, auf eine mediastinale Veränderung hinweisen (Uffmann et al. 1998). Darüber hinaus wird in der Thoraxröntgenuntersuchung eine verbreiterte obere Mediastinalweite von $>8 \mathrm{~cm}$ und ein Mediastinum/Thorax-Quotient von $>0,25$ als hochgradig lazerationsverdächtig angesehen (Marsh und Sturm 1976, Müller et al. 2001).

Die hier vorgestellte Studie hat die Aussagekraft und Repräsentativität der Thoraxröntgenuntersuchung hinsichtlich vorliegender traumatisch bedingter Mediastinalverletzungen hinterfragt.

Die Messwerte 1 und 2 der berechneten Mediastinum/Thorax-Quotienten zeigen eine nur geringe Abweichung der Durchschnittswerte von M1 0,298 und M2 0,307 mit minimaler Standardabweichungen sowie einem nicht signifikanten Unterschied. Daraus lässt sich ableiten, dass sowohl der auf Herzklappenebene (M1) als auch der auf Höhe des Aortenbogens (M2) ermittelte Mediastinum/Thorax-Quotient den von Müller et al. (2001) genannten Grenz-Quotienten von >0,25 deutlich überschreiten und formal als pathologisch verbreitert anzusehen sind. Dies würde bedeuten, dass fast alle über den Schockraum eingelieferten Patienten eine auffällige Thoraxröntgenuntersuchung aufweisen. Bei der deutschlandweit allgemein eher geringen Häufigkeit mediastinaler Verletzungen erscheint dies als suspekt und wirft Fragen bezüglich des gewählten Grenzwertes auf. 
Um eine eigenständige Aussage hinsichtlich eines pathologischen Grenzwertes treffen zu können, erfolgte die Aufteilung aller Messwerte in die Untersuchungsgruppen Auffällig und Unauffällig anhand gesichert vorliegender Mediastinalverletzungen. Eine weitere Messwertaufteilung der Untersuchungsgruppen anhand des Geschlechtes wurde bei vergleichbarer Messwertverteilung und nicht signifikantem Unterschied als wenig sinnvoll erachtet (siehe Abbildung 3.2).

Bei der Aufteilung in die Untersuchungsgruppen konnten bei der Patientengruppe Auffällig weder höhere Maximalwerte des Mediastinum/Thorax-Quotienten noch ein signifikanter Unterschied zur Patientengruppe Unauffällig festgestellt werden. Somit kann weder bei extrem hohen noch bei vom allgemein gültigen Grenzwert $>0,25$ abweichenden Mediastinalquotienten automatisch auf eine vorliegende Mediastinalverletzung geschlossen werden (Müller et al. 2001).

Zur weiteren Analyse erfolgte die Unterteilung der einzelnen Messwerte 1 und 2 anhand definierter Mediastinum/Thorax-Quotienten-Grenzwerte unter Berücksichtigung tatsächlich vorliegender Mediastinalverletzungen. Dabei zeigt sich für Messwert 1 bei einem Mediastinum/Thorax-Quotienten von 0,30 und für Messwert 2 bei 0,28 eine vertretbare, aber eher schwache maximale Sensitivität von 70\% (M1) und 63\% (M2). Gleichzeitig konnte eine nicht zufriedenstellende Spezifität von 57\% für M1 und 38\% für M2 festgestellt werden. Eine solche Spezifität ist vergleichbar mit einer 50prozentigen Ratewahrscheinlichkeit, bei der eindeutig zu viele unauffällige Patienten fälschlicherweise als auffällig detektiert werden.

Aufgrund der nicht zufriedenstellenden Relation zwischen Sensitivität und Spezifität sowie der nicht aussagekräftigen Maximalwerte des Mediastinum/Thorax-Quotienten ist eine valide Beurteilung pathologischer Mediastinalverhältnisse durch die Thoraxröntgenuntersuchung im Schockraum als äußerst fragwürdig anzusehen.

Die von Seltzer et al. (1981) veröffentlichte Studie zu Aortenrupturen bei stumpfen Thoraxtraumen berücksichtigt ebenfalls in der Thoraxröntgenuntersuchung ermittelte Mediastinum/Thorax-Quotienten. Bei einem pathologischen Grenzwert von 0,28 konnte eine maximale Sensitivität von $85 \%$ und eine Spezifität von $100 \%$ detektiert werden (Seltzer et al. 1981). Hinterfragt man diese doch deutlich höhere Spezifität und Sensitivität, so stellen die geringere Personenanzahl, die passend gewählten kleineren Kontrollgruppen, aber auch die schlechtere Bildqualität und technischen Möglichkeiten von 1981 Ergebnis-beeinflussende Faktoren dar. Auch spielt die Variation der 
Entfernung zwischen Röntgenröhre und zu durchleuchtendem Objekt eine entscheidende Rolle. Diese wurde zwar von Seltzer et al. (1981) in jeder Untersuchungsgruppe intern standardisiert angewandt, variiert jedoch zwischen den einzelnen Vergleichsgruppen. Dies schwächt die Aussagekraft des Gruppenvergleichs und der Testgenauigkeit deutlich ab. Darüber hinaus gilt es bei dem von Seltzer et al. (1981) in Bezug auf die 2. Kontrollgruppe detektierten signifikanten Unterschied zu berücksichtigen, dass sich diese ausschließlich aus unauffälligen, nicht hospitalisierten Patienten zusammensetzt. Außerdem wurden die Thoraxröntgenuntersuchungen nicht im Liegen, sondern stehend in p.a.-Projektion durchgeführt und sind somit nicht auf die Schockraumsituation übertragbar. Zusätzlich zeigt die von Hehir et al. (1990) angefertigte Studie, dass die im Liegen und Stehen durchgeführte Thoraxröntgenuntersuchung sich hinsichtlich der Sensitivität zur Detektion von Thoraxverletzungen um 20\% (liegend 58,3\%; stehend 78,7\%) zugunsten der Aufnahmen im Stehen unterscheidet. Somit ist ein Vergleich von Patienten mit unterschiedlich durchgeführten Thoraxröntgenuntersuchungen als nicht repräsentativ zu werten.

Auch Marnocha et al. (1984) beschreiben in einer retrospektiven Studie keinen zufriedenstellenden klinisch brauchbaren Zusammenhang der Sensitivität und Spezifität erhobener Mediastinum/Thorax-Quotienten und vorliegenden Aortenrupturen. Somit können weder mit der hier angefertigten noch mit der von Marnocha et al. (1984) durchgeführten Untersuchung die von Seltzer et al. (1981) erhobenen Ergebnisse nachvollzogen werden.

Die Tatsache, dass sowohl 1984, damals ohne CT, bis heute allenfalls eine vage Aussage mittels Thoraxröntgenuntersuchung möglich ist, verdeutlicht den geringen Nutzen des Röntgen und unterstreicht den hohen Stellenwert des CT im Schockraum zur Detektion von Thoraxverletzungen.

Auch Uffmann et al. (1998) verweisen unter Berücksichtigung der von Woodring 1990 angefertigten Sammlung aus 52 Papern zu stumpfen thorakalen Gefäßverletzungen (1953 bis 1990) darauf, dass eine Mediastinalverbreiterung im Röntgen nur eine sehr geringe Sensitivität von 10 bis $20 \%$ aufweist.

Dies deckt sich auch mit den Aussagen von Raptopoulos et al. (1992), Hunink und Bos (1995) und Ho et al. (2002), dass die Sensitivität einer Verbreiterung des Mediastinums in der Thoraxröntgenuntersuchung unzureichend für eine initiale Beurteilung im 
Schockraum ist. Deshalb werden sensitivere Verfahren wie beispielsweise das CT als Bildgebung befürwortet.

Auch die American Association for the Surgery of Trauma (AAST) betont den klaren Vorteil des CT zur Detektion von mediastinalen Gefäßverletzungen bei stumpfen Thoraxtraumen. Dabei stützt sich die AAST auf eine prospektiv multizentrische Vergleichsstudie, ohne die Thoraxröntgenuntersuchung als mögliche Detektionsmethode überhaupt zu berücksichtigen (Demetriades et al. 2008).

Tabelle 4.1: Autorenübersicht mit Aussagen zu Thoraxröntgenuntersuchung-bezogener Testgenauigkeit.

\begin{tabular}{|c|c|c|c|}
\hline Autor & Jahr & $\begin{array}{l}\text { Patienten- } \\
\text { anzahl }\end{array}$ & Aussage \\
\hline Marnocha et al. & 1984 & 54 & $\begin{array}{l}\text { Der Mediastinum/Thorax-Quotient aus } \\
\text { Thoraxröntgenuntersuchung besitzt eine } \\
\text { unzureichende Sensitivität und Spezifität, } \\
\text { um eine Aortenruptur zu bestätigen oder } \\
\text { auszuschließen. }\end{array}$ \\
\hline Hehir et al. & 1990 & 100 & $\begin{array}{l}\text { Sensitivitätsunterschied von } 20 \% \text { der } \\
\text { Thoraxröntgenuntersuchung zur Detektion } \\
\text { von Thoraxverletzungen zugunsten der } \\
\text { Aufnahmen im Stehen. }\end{array}$ \\
\hline Raptopoulos et al. & 1992 & 326 & \multirow{3}{*}{$\begin{array}{l}\text { Sensitivität einer Verbreiterung in der } \\
\text { Thoraxröntgenuntersuchung des Mediasti- } \\
\text { nums ist als unzureichend zu werten. }\end{array}$} \\
\hline Hunink und Bos & 1995 & 462 & \\
\hline Ho et al. & 2002 & 77 & \\
\hline Uffmann et al. & $\begin{array}{c}1953 \\
\text { bis } \\
1990\end{array}$ & 656 & $\begin{array}{l}\text { Mediastinalverbreiterung in der Thorax- } \\
\text { röntgenuntersuchung hat eine sehr geringe } \\
\text { Sensitivität. }\end{array}$ \\
\hline
\end{tabular}

In der hier vorliegenden Studie wurden, um mögliche Messungenauigkeiten und fehlerhafte Messergebnisse auszuschließen, die Mediastinalverhältnisse an zwei unterschiedlichen Stellen ermittelt. Hierbei konnte auf beiden Höhen ein nur geringfügig voneinander abweichender Durchschnittswert von 0,298 $\pm 0,006$ für M1 und 
0,307 $\pm 0,006$ für M2 festgestellt werden. Dies bestätigt die Vergleichbarkeit und schließt gleichzeitig große Messungenauigkeiten aus. Geräte-bezogene Abweichungen sind aufgrund während des Untersuchungszeitraums unveränderter Röntgenapparatur und gleichem Untersuchungsablauf als unbedeutend $\mathrm{zu}$ erachten. Kritisch ist jedoch die Zusammensetzung der Untersuchungsgruppen zu betrachten. Eine weitere Aufteilung des Patientenkollektivs in mehrere gleich große Kleingruppen unter Berücksichtigung von Alter, Körperstatur und Grunderkrankungen wäre bei einer erneuten Testung empfehlenswert.

Anhand der durchgeführten Studie konnte gezeigt werden, dass die Ausmessung der Thoraxröntgenuntersuchung von Schockraumpatienten weder eine sichere Diagnose noch einen sicheren Ausschluss mediastinaler Verletzungen zulässt. Die Thoraxröntgenuntersuchung ist hinsichtlich der Fragestellung pathologischer Verbreiterungen des Mediastinums in der Primärphase des Schwerverletztenmanagements im Schockraum nicht aussagekräftig und daher als nicht mehr zeitgemäß anzusehen. Diese Feststellung wird durch die 2016 erschienene Leitlinie Polytrauma bestätigt. Es wird angeraten die Thoraxröntgenuntersuchung im Schockraum nicht mehr primär sondern nur bei Nichtverfügbarkeit des CT einzusetzen (Leitlinie Polytrauma 2016).

\subsection{Prädiktoren mediastinaler Verletzungen}

Mediastinale Verletzungen können auf vielfältige Weise entstehen, wobei für traumatische Ursachen hauptsächlich stumpfe, direkte Anprall- bzw. Dezellerationstraumen verantwortlich sind (Linsenmaier und Schueller 2010). Bereits 1960 wurde in einer Publikation von Zehnder eine deutliche Zukunftsprognose gegeben, dass bei zunehmend motorisierter Verkehrsdichte die unfall-bedingten traumatischen Aortenrupturen an Bedeutung gewinnen werden. Auch Hermanutz und Bücheler (1974) beschreiben, dass mit zunehmender Schwere der Verkehrsunfälle die Häufigkeit von Aortenrupturen zunimmt. 1991 wurde dann explizit der Zusammenhang von PKWUnfällen und Aortentraumen durch Gurt- und Lenkradverletzung als gesicherter Unfallmechanismus vorgestellt (Frobenius 1991). Wie im Einleitungsteil (siehe Abbildung 1.1) dargestellt, gehören Verkehrsunfälle mit zu den häufigsten Unfallursachen über den Schockraum eingelieferter Patienten. Deswegen ist die Auseinandersetzung mit direkt assoziierten Krankheitsbildern, wie traumatischen 
Mediastinalverletzungen, indiziert und stellt eine aktuelle Problematik dar (TraumaRegister DGU® 2017, TraumaRegister DGU® 2018).

Um Prädiktoren mediastinaler Verletzungen zu evaluieren, wurden personen-bezogene Daten, Unfallmechanismus, Schweregrad der Thoraxverletzung, Unfallort und Schockraum Daten, Trauma-Scores und Letalität, mit dem Ziel einer möglichst schnellen Therapieeinleitung analysiert.

\subsubsection{Patientenkollektiv und Herzsilhouette}

Patienten-bezogene Daten wie Alter und Geschlecht stellen Standardparameter dar, die auch im Jahresbericht der DGU $®$ veröffentlicht werden. Die Geschlechterverteilung des untersuchten Patientenkollektivs zeigt mit 77\% ein männerlastiges Patientenkollektiv im Alter von $49 \pm 21$ Jahren. Diese Geschlechterverteilung stimmt laut Jahresbericht des TraumaRegister DGU® (2018) mit den deutschlandweit dokumentierten Schockraumpatienten (70\% männlich) nahezu überein. Die Signifikanztestung der Geschlechterverteilung zeigt keinen signifikanten Unterschied, weshalb bei Verdacht auf mediastinale Verletzungen das Geschlecht nicht als Entscheidungsparameter zur Therapieeinleitung herangezogen werden kann.

Bei Blutungen von im Mediastinum liegenden Gefäßen kann es zur Ausbildung eines Hämatothorax oder Perikarderguss kommen (Heberer 1968). Zusätzliches Blutvolumen im Mediastinum kann $\mathrm{zu}$ einem pathologischen Thoraxbild im Röntgen führen (Freyschmidt und Hahn 2007). Bei der Untersuchungsgruppe Auffällig zeigen sich trotz einer durchschnittlich etwa $3 \mathrm{~mm}$ breiteren Herzsilhouette prozentual weniger Patienten mit einem pathologischen Herz/Thorax-Quotient (22\% Auffällig zu 26\% Unauffällig). Zusammen mit den negativen Ergebnissen der Signifikanztestung lässt sich folgern, dass weder über die Herzsilhouettengröße noch den Herz/Thorax-Quotienten auf eine mediastinale Verletzung geschlossen werden kann.

\subsubsection{Grad der Thoraxverletzung}

Bei nahezu jedem über den Schockraum eingelieferten Patienten wird der Verletzungsgrad der verschiedenen Körperregionen dokumentiert. Im Jahresbericht des TraumaRegister DGU® (2018) zeigen sich Thoraxverletzungen im Zeitraum von 2015 bis 2017 mit 45\% deutschlandweit als zweit häufigstes Verletzungsmuster von Schockraumpatienten, in Bezug auf das Basis-Kollektiv. 
Die Untersuchungsgruppen wurden hinsichtlich der in vier Schweregrade (keine, leicht, mittelschwer, schwer) eingeteilten Thoraxverletzungen miteinander verglichen. Dabei zeigt sich eine Korrelation zwischen der Verletzungsschwere und der Häufigkeit mediastinaler Verletzungen bei zusätzlich signifikantem Unterschied. Somit kann davon ausgegangen werden, dass je schwerer die Thoraxverletzung ist, desto mehr mediastinale Verletzung liegen vor. Die damit assoziierte negative Patientenprognose wird in der Literatur mit der ebenfalls zunehmenden Wahrscheinlichkeit einer Lungenkontusion in Verbindung gebracht (Peters et al. 2007). Die Mitverletzung der Lunge trägt dabei zur hohen und oftmals ARDS vermittelten Letalität stumpfer Thoraxtraumen bei (Regel und Pape 2000, Peters et al. 2007). Dies unterstreicht den letalitätssteigernden und sich negativ auf die Prognose des Patienten auswirkenden Einfluss von Thoraxverletzungen. Somit ist es gerechtfertigt den Schweregrad der Thoraxverletzung als wichtigen Prädiktor mediastinaler Verletzungen zu berücksichtigen und ihn bei der Entscheidung einer frühzeitigen Therapieeinleitung mit einzubeziehen.

\subsubsection{Unfallmechanismus und Ursache}

Bei Aortenverletzungen spielen Anprall- bzw. Dezelerationstraumen, wie sie bei Verkehrsunfällen und Sturzgeschehen vorkommen, eine wichtige Rolle (Zehnder 1974). Trotz zunehmender Motorisierung und Verkehrsdichte mit steigender Gefahr und Häufigkeit mediastinaler Verletzungen, konnte beim Unfallmechanismus Verkehrsunfall, zwischen beiden Untersuchungsgruppen Auffällig und Unauffällig kein signifikanter Unterschied festgestellt werden. Verkehrsunfälle stellen zwar laut Jahresbericht des TraumaRegister $\mathrm{DGU}^{\circledR}$ (2018) deutschlandweit eine führende Unfallursache von Schockraumpatienten dar, sind aber nicht automatisch mit einer mediastinalen Verletzung assoziiert. Eine frühzeitige Therapieeinleitung allein aufgrund des typischen Unfallmechanismus ist nicht zu rechtfertigen.

\subsubsection{Vitalparameter Unfallort und Schockraum}

Vitalparameter sind standardmäßig präklinisch und klinisch erhobene Messwerte, die zur Überwachung des Patienten benutzt werden.

Bezogen auf den RR spricht man definitionsgemäß ab einem systolischem $R R<90$ mmHg und vermindertem Herz-Zeit-Index von $<2,2$ von einem kardiogenen Schock (Adams et al. 2001). Ebenso ist bei vorliegender Verletzung des Mediastinums eine 
kardiale Einschränkung denkbar. Hinsichtlich der berechneten Durchschnittswerte zeigten sich bei beiden Untersuchungsgruppen sowohl präklinisch als auch im Schockraum keine Wert $<120 \mathrm{mmHg}$. Bei der Untersuchungsgruppe Auffällig liegt ein insgesamt zu beiden Erhebungszeitpunkten durchschnittlich geringerer systolischer RR vor, wobei nur $<25 \%$ der Werte $<100 \mathrm{mmHg}$ liegen. Deshalb ist nicht generell von einem Schockzustand mit kardialer Einschränkung auszugehen. Der sowohl am Unfallort als auch im Schockraum nicht signifikante Unterschied beider Untersuchungsgruppen zeigt, dass der systolische RR zu keinem Zeitpunkt eine eindeutige Vorhersagekraft hinsichtlich mediastinaler Verletzungen besitzt und deshalb keine Relevanz bei der Einleitung therapeutischer Maßnahmen besitzt.

Eine Beeinträchtigung der kardialen Auswurfleistung kann zu einer kompensatorischen Veränderung der Herzfrequenz führen. Tachykarde Herzfrequenzen durch sympathoadrenerge Gegenregulation können als Kompensationsmechanismen des Herzens angesehen werden, um Durchblutung und Sauerstoffversorgung mittels gesteigertem Herzzeitvolumen aufrecht zu erhalten (Brambrink und Noppens 2001). Am Unfallort zeigten sich bei der Untersuchungsgruppe Auffällig durchschnittlich tachykarde HF Werte von 101/min. Ursachen hierfür können sowohl eine Einschränkung der kardialen Auswurfleistung als auch die durch Aufregung und Stress am Unfallort-bedingte massiv erhöhte adrenerge Hormonausschüttung sein (Sefrin et al. 1980). Die zu beiden Erhebungszeitpunkten deutlich höheren Maximalwerte der HF der Untersuchungsgruppe Unauffällig zeigen, dass anhand von extrem von der Norm abweichender Werte nicht automatisch auf eine vorliegende Mediastinalverletzung geschlossen werden kann.

Fasst man zusammen, so zeigt sich aufgrund des nur knapp oberhalb der Tachykardiegrenze liegenden Durchschnittswert der Patientengruppe Auffällig sowie fehlende bradykarde HF-Werte und nicht signifikanten Unterschied, dass die HF weder eine Relevanz als Prädiktor zur Früherkennung mediastinaler Verletzungen noch zur frühzeitigen Therapieeinleitung besitzt.

Die Sauerstoffsättigung ist ein von der Auswurfleistung und Funktionstüchtigkeit des Herzens abhängiger Parameter zur Darstellung des peripheren arteriellen Sauerstoffgehalts im Blut. Der sigmoide Verlauf der Sauerstoffbindungskurve zeigt ab einer peripheren $\mathrm{SpO}_{2}$ Konzentration von $<90 \%$ einen rapiden Abfall der Sauerstoffsättigung des Blutes, weshalb 90\% als Hypoxiegrenze definiert wird (Lindemann 1998). 
Die zu beiden Erhebungszeitpunkten durchschnittlich niedrigeren und am Unfallort sogar unterhalb der Hypoxiegrenze befindlichen $\mathrm{SpO}_{2}$-Werte der Untersuchungsgruppe Auffällig könnten entweder auf eine verminderte kardiale Auswurfleistung oder auf eine pulmonal eingeschränkte Oxygenierung hinweisen (Gestel und Teschler 2015). Dennoch müssen die ermittelten $\mathrm{SpO}_{2}$-Werte allgemein hinterfragt werden, da die präklinisch und klinisch angewandten $\mathrm{SpO}_{2}$-Sensoren eine starke kälte-, höhenmeterund verschmutzungs-bezogene Störanfälligkeit aufweisen (Storck und Girwidz 2016, Domej et al. 2011). Unter Berücksichtigung der Störanfälligkeit der $\mathrm{SpO}_{2}$-Sensoren und des nicht signifikanten Unterschiedes der Untersuchungsgruppen ist die peripher gemessene Sauerstoffkonzentration als nicht richtungsweisend hinsichtlich mediastinaler Verletzungen und einer frühzeitig einzuleitenden Therapie zu werten.

\subsubsection{Invasive Maßnahmen und Medikamente}

Die Schockraumdokumentation beinhaltet neben den Patientendaten und Vitalparametern auch durchgeführte Maßnahmen und die Medikamentengabe.

Die Intubation und die Anlage einer Thoraxdrainage stellen invasive Maßnahmen am Unfallort und im Schockraum dar. Die zu beiden Zeitpunkten mit 47\% gleichbleibenden und um 9\% am Unfallort und 36\% im Schockraum höheren Intubationszahlen der Untersuchungsgruppe Auffällig zeugen von dem hohen Stellenwert der pulmonalen Unterstützung bei Patienten mit mediastinalen Verletzungen. Dies befürwortet auch die prospektive Studie von Trupka et al. (1995), in der eine signifikante Reduktion der Letalitätsrate schwerer Thoraxtraumen durch frühzeitige Intubation festgestellt werden konnte. Zusätzlich konnte hinsichtlich des Intubationsstatus im Schockraum ein signifikanter Unterschied festgestellt werden.

Der Thoraxdrainagestatus ist bei der Untersuchungsgruppe Auffällig prozentual gesehen am Unfallort um das Fünffache und im Schockraum um das Sechsfache höher als bei der Vergleichsgruppe Unauffällig. Diese deutlich höhere Interventionsrate der Untersuchungsgruppe Auffällig unterstreicht den hohen Stellenwert der thorax- und lungenentlastenden Maßnahme bei vorliegenden Mediastinalverletzungen. Zusätzliche das Mediastinum belastende Zustände wie ein Pneumo-/Hämato-/ oder Spannungspneumothorax bedürfen einer sofortigen Behandlung (Kopp et al. 2008). Außerdem können laut Kopp et al. (2008) über die Menge ( $>500 \mathrm{ml}$ Blut) und die Beschaffenheit des Drainageinhaltes Rückschlüsse auf zentrale Gefäßverletzungen gezogen werden. 
Die Signifikanztestung zeigt für den im Schockraum erhobenen Thoraxdrainagestatus einen signifikanten Unterschied.

Unter Berücksichtigung der Auswertung beider invasiver Maßnahmen (Intubation und Thoraxdrainage) spiegeln die deutlich höheren Prozentzahlen durchgeführter Interventionen der Untersuchungsgruppe Auffällig deren hohen Stellenwert bei Mediastinalverletzungen wider. Aufgrund des signifikanten Unterschiedes im Schockraum kann die Notwendigkeit einer Intubation oder einer Thoraxdrainage als Hinweis auf das Vorliegen einer mediastinalen Verletzung fungieren. Deshalb sollten weitere Untersuchungen, beispielsweise ein CT-Thorax zur Analyse des Mediastinums folgen, um eine frühzeitige Therapieeinleitung genauestens zu evaluieren.

Bei der Katecholamin-Gabe zeigt sich in der vorliegenden Studie bei der Untersuchungsgruppe Auffällig zu beiden Erhebungszeitpunkten mit 40\% eine vierfach höhere Prozentzahl an Katecholamin-bedürftigen Patienten als bei der Vergleichsgruppe Unauffällig (10\% Patienten mit Katecholaminen). Präklinisch weist die Signifikanztestung einen minimal $\left(10^{-6}\right)$ unterhalb der Signifikanzgrenze von 0,002381 liegenden $\mathrm{p}$-Wert auf. Problematisch stellt sich die Zuordnung der verabreichten Katecholaminart dar, was anhand der Dokumentation nicht nachvollziehbar ist. Zusammenfassend ist aufgrund der nur schwachen Signifikanz und der nicht nachvollziehbaren Katecholamin-Substanzgruppe der Katecholaminstatus als ein lediglich schwacher und vernachlässigbarer Hinweis auf eine vorliegende mediastinale Verletzung zu werten.

Eine weiterführende Studie zur Untersuchung der Beziehung von Mediastinalverletzungen und dem Einsatz verschiedener Katecholamin-Substanzgruppen in Form einer prospektiven Verlaufsstudie würde sich zur genaueren Evaluierung des vorliegenden Untersuchungsergebnisses eignen.

\subsubsection{Trauma-Scores}

Der NISS und RISC-II-Score dienen der Klassifizierung und Beurteilung von Verletzungsschwere und Überlebenswahrscheinlichkeit schwerverletzter Patienten.

Der durchschnittliche NISS der Patientengruppe Auffällig ist mit 39 Punkten um elf Punkte höher und beim 3. Quantil sogar um 16 Punkte höher als bei der Vergleichsgruppe Unauffällig. Auch hinsichtlich des niedrigsten NISS zeigt sich bei der Patientengruppe Auffällig mit 12 Punkten eine 9 Punkte höhere Verletzungsschwere 
verglichen mit der Untersuchungsgruppe Unauffällig. Sowohl die höhere 75\% Grenze (3. Quantil) als auch der deutlich über 20 Punkte liegende mittlere NISS der Untersuchungsgruppe Auffällig, spiegeln im deutschlandweiten Schockraumpatientenvergleich von 2015 bis 2017 (durchschnittlich 18 NISS Punkte) die gravierende Verletzungsschwere und den dringlichen Therapiebedarf mediastinaler Verletzungen wider (TraumaRegister DGUß 2018).

Der RISC-II-Score der Patientengruppe Auffällig weist mit durchschnittlich 73\% eine um $15 \%$ niedrigere und somit deutlich reduziertere Überlebenswahrscheinlichkeit auf. Auch das 1. Quantil (<35.3\%) bestätigt, dass bei Untersuchungsgruppe Auffällig prozentual mehr Patienten eine deutlich schlechtere Überlebenswahrscheinlichkeit aufweisen als die der Patientengruppe Unauffällig (1.Quantil bei 90\%).

Die p-Werte beider Trauma-Scores (NISS und RISC-II) zeigen keine signifikanten Unterschiede, weshalb sie zwar individuell stark aussagekräftig sind, aber sich nicht als allgemeine Prädiktoren mediastinaler Verletzungen oder zur Evaluation einer frühzeitigen Therapie eignen.

\subsubsection{Letalität}

Die Letalität des gesamten Patientenkollektivs liegt bei 8\%. Dies ist vergleichbar mit den im Jahresbericht des TraumaRegister DGU® (2018) veröffentlichten Werten der deutschlandweiten Sterberate von 10,8\% aller im TraumaRegister DGUß gelisteten Schockraumpatienten. Die Letalität stellt einen praxisrelevanten Parameter dar, um Kliniken untereinander zu vergleichen. Unterteilt in die Untersuchungsgruppen zeigt sich prozentual eine mit 15\% fast doppelt so hohe Letalitätsrate der Patienten mit mediastinaler Verletzung (Gruppe Auffällig). Dies veranschaulicht trotz nicht signifikantem Unterschied beider Untersuchungsgruppen den dringlichen Behandlungsbedarf und die Notwendigkeit einer möglichst frühen Behandlung zur Senkung der Letalität.

Mediastinale Gefäßverletzungen gehen oftmals ohne äußere Verletzungen einher und werden deshalb vom Notfallpersonal nicht sofort erkannt (Kirsh et al. 1976, Barcia und Livoni 1983). Deshalb sind Untersuchungen am Unfallort bzw. im Schockraum und die initiale Bildgebung im Schockraum wichtige Bestandteile der Diagnostik mediastinaler Verletzungen. 
Den Untersuchungen zufolge lassen weder patienten-bezogene Daten, der Unfallmechanismus noch präklinisch durchgeführte Maßnahmen oder erhobene Vitalparameter einen Rückschluss auf vorliegende mediastinale Verletzungen zu. Dies beschreibt auch die Studie von Zehnder (1960) in der postuliert wird, dass sich die klinischen Symptome beispielsweise einer Aortenruptur sehr variabel darstellen können und sich nicht zur Sicherung der Diagnose eignen. Das American College of Surgeons Committee on Trauma (ACS COT) geht sogar so weit, die deutliche Over Triage Rate von 30 bis 50\% auf initial am Unfallort erhobene Parameter zurückzuführen, was die präklinisch erhobenen Parameter nicht nur bei mediastinalen Verletzungen, sondern generell in Zweifel zieht (Hinkelbein et al. 2013).

Die von Blackmore et al. (2000) angefertigte Studie zur Optimierung der Bildgebungsstrategie und Ermittlung der Risikowahrscheinlichkeit traumatischer Aortenverletzungen befasst sich mit vermeintlich signifikanten Parametern wie Alter, Hypotension ( $<90 \mathrm{mmHg}$ ) und vorliegendem Pneumothorax. Dabei stimmen die hier als signifikant detektierten Unterschiede hinsichtlich vorliegendem Pneumothorax überein. Die sowohl von Blackmore et al. (2000) als auch von Kram et al. (1987) berücksichtigte Hypotension von $<90 \mathrm{mmHg}$ kann anhand der hier erhobenen Daten wegen fehlender Unterteilung und Signifikanz der RR-Werte weder bestätigt noch widerlegt werden. Dennoch ist es ein interessanter Ansatzpunkt für weitergehende Forschung und könnte im Rahmen einer erneut retrospektiven Untersuchung getestet werden.

Kritisch müssen die Dokumentations- und Übertragungsprozesse des TraumaRegister DGU ${ }^{\circledR}$ betrachtet werden. Die Daten werden primär per Hand im Schockraum erhoben und manuell in die Online-Datenbank des Traumaregisters eingepflegt, was eine potentielle Fehlerquelle darstellt. Außerdem können die hektische Schockraumatmosphäre und der starke Zeitdruck im Schockraum zu einer lückenhaften Datenerhebung führen. Deshalb stehen bei den Untersuchungsgruppen Auffällig und Unauffällig teilweise nicht alle 27 bzw. 240 Patienten als Vergleichspopulation zur Verfügung. Neben der Datenerhebung muss auch die Auswertung kritisch betrachtet werden. Die Signifikanztestung des Patientenkollektivs erfolgte anhand variierender Patientenzahlen die auf Dokumentationslücken oder Übertragungsfehler zurückzuführen sind. Die Anpassung des p-Wertes auf 0,017 bzw. 0,002381 zielt darauf ab, die Wahrscheinlichkeit zufällig signifikanter Unterschiede zu minimieren. Dabei wurde auch die Aussagekraft der Signifikanzen anhand der Differenz zur p-Wertgrenze berücksichtigt. Kritisch ist auch die unterschiedliche Größe der Untersuchungsgruppen 
zu betrachten wodurch die einzelnen Signifikanzen in ihrer Aussagekraft abgeschwächt werden.

Beim Jahresbericht des TraumaRegister DGUß, der als Bezugsgröße deutschlandweit dokumentierter Schockraumpatienten dient, gilt es zu berücksichtigen, dass lediglich ein als Basis-Kollektiv definierter Patientenstamm dargestellt wird. Dieses Kollektiv klammert leichtverletzte Patienten aus und setzt sich aus schwerverletzten Patienten mit einem MAIS 3+ sowie allen Patienten mit einem MAIS 2, die entweder verstorben sind oder auf Intensivstation lagen, zusammen (TraumaRegister DGU $® 2018$ ).

Zusammenfassend ist eine typische Symptomatik für mediastinale Verletzungen nur schwer zu beschreiben. Bezogen auf Aortenverletzungen ist der klinische Befund abhängig vom Ausmaß der Unterbrechung der Aortenwand, der Schwere des Thoraxtrauma und den nicht thorakalen Begleitverletzungen (Kopp et al. 2008). Patienten-bezogene Daten, der Unfallmechanismus und präklinisch erhobene Parameter konnten hierbei keine aussagekräftigen Werte liefern, die auf eine Mediastinalverletzung hinweisen.

Klinisch im Schockraum zeigen sich hingegen Parameter, wie der Schweregrad der Thoraxverletzung, der Intubations- und Thoraxdrainagestatus, als richtungsweisend. Diese sind mit erhöhter Wahrscheinlichkeit mit mediastinalen Verletzungen vergesellschaftet, weshalb bei vorliegendem Befunden ein besonderes Augenmerk auf die weitere diagnostische Abklärung gelegt und an eine frühzeitige Therapieeinleitung gedacht werden sollte.

\subsection{Aussagekraft von eFAST und Thoraxröntgenuntersuchung zur Pneumo- und Hämatothorax-Diagnostik im Schockraum}

Die Thoraxsonographie ist ein diagnostisches Mittel zur Detektion thorakaler Verletzungen mit der Möglichkeit sofort therapeutische Schritte zu veranlassen (Kreuter et al. 2012). Beispielsweise führt in den Pleuraspalt eingedrungene Luft zu einem fehlenden Lungengleiten in der Thoraxsonographie (Michels et al. 2014). Auch die Thoraxröntgenuntersuchung wird zur Diagnose eines Pneumothorax eingesetzt. Pathologien fallen in Form sichtbarer Pleuralinien, vermehrter Transparenz und fehlender Gefäßzeichnung lateral der kollabierten Lunge auf (Diederich und Wormanns 2009). 
Die angefertigte Studie zielt auf eine genaue Differenzierung der Aussagekraft von eFAST und Thoraxröntgenuntersuchung ab. Dabei hat sich eine bei $80 \%$ liegende und vierfach höhere Sensitivität des eFAST gezeigt, wodurch die Überlegenheit des eFAST verglichen mit der Thoraxröntgenuntersuchung (18\% Sensitivität) verdeutlicht wird. Die bei beiden Untersuchungen mit 100\% identische Spezifität ist zu vernachlässigen, da sie sich auf die Detektion unauffälliger Patienten bezieht und keine Relevanz für die Fragestellung nach einem Pneumo-/Hämatothorax besitzt.

Die Studie von Kirkpatrick et al. (2004) konnte bei vergleichbarer Spezifität von 99,2\% eine bei 49\% liegende und insgesamt 29\% höhere Sensitivität des eFAST, verglichen mit der Sensitivität der Thoraxröntgenuntersuchung, feststellen. Auch diese Daten zeigen die Überlegenheit des eFAST in der Pneumo-/Hämatothorax-Diagnostik. Der deutliche Sensitivitätsunterschied des eFAST im Vergleich zur hier durchgeführten Studie kann durch den technischen Fortschritt heutiger Ultraschallgeräte oder möglicherweise fehlende Untersucherexpertise erklärt werden. Hinsichtlich der Studiendurchführung zeigen sich bis auf eine längere Studiendauer seitens der Studie von Kirkpatrick et al. (2004) deutliche Parallelen zur hier veröffentlichten Untersuchung in Durchführung, Ablauf, Patientenzahl und -kollektiv.

In der von Zhang et al. (2006) publizierten prospektiven Studie zeigt sich mit 27,6\% eine deutlich niedrigere Sensitivität der konventionellen Thoraxröntgenuntersuchung im Vergleich zur 86,2\%-igen Sensitivität des eFAST. Bei vergleichbarem Untersuchungsaufbau und -durchführung unterstreichen diese Ergebnisse die deutlich überlegene Aussagekraft des eFAST zur Pneumo-/Hämatothorax-Detektion. Zusätzlich nennt Zhang et al. (2006) eine signifikante Zeitersparnis als weiteren Vorteil des eFAST.

Auch von Nagarsheth und Kurek (2011) wird im American Surgeon die Empfehlung der Nutzung des eFAST aufgrund der höheren Sensitivität gegeben. Diese Empfehlung basiert auf einer mit 81,8\% insgesamt 50\% höheren Sensitivität des eFAST im Vergleich zur Thoraxröntgenuntersuchung (31,8\%) (Nagarsheth und Kurek 2011).

Laut Hyacinthe et al. (2012) hat sich aufgrund hoher Sensitivität, schneller Durchführbarkeit und leichter Erreichbarkeit des eFAST im Schockraum die Beurteilung von Thoraxtraumen verbessert. Deshalb sollten auch hämodynamisch- und respiratorisch instabile Patienten einer Thoraxsonographie unterzogen werden. Dieses Zugeständnis bei maximal schwerverletzten Patienten unterstreicht den hohen und 
aussagekräftigen Stellenwert des eFAST in der initialen Schockraumbehandlung und bestätigt die hier veröffentlichten Untersuchungserbnisse.

In der Literatur zeigen sich schwankende Werte der eFAST Sensitivität, die durch eine unterschiedlich stark ausgeprägte Luftansammlung im Pleuraspalt, beispielsweise bei einem Spitzen- oder Mantelpneumothorax, zu erklären sind (Kirkpatrick et al. 2004). Die Detektion solch minimaler Luftmengen im Pleuraspalt ist oftmals mit dem eFAST nicht möglich und nur durch die als Goldstandard angesehene Computertomographie feststellbar (Reissig und Kroegel 2005). Ebenso kann sich ein Pneumothorax im Verlauf größenprogredient darstellen, wodurch sich die Luftansammlung erst zu einem späteren Zeitpunkt demaskiert.

Trotz schwankender Prozentzahlen stimmen die Studien in der Erkenntnis einer deutlich höheren Sensitivität der Thoraxsonographie überein und teilen das Untersuchungsergebnis der hier durchgeführten Studie. Somit ist das eFAST der konventionellen Thoraxröntgenuntersuchung zur Pneumo-/Hämatothorax-Diagnostik im Schockraum vorzuziehen.

Die wechselnde Durchführung und Auswertung von eFAST, Thoraxröntgenuntersuchung und CT durch Personal der Unfallchirurgie bzw. Radiologie sollte kritisch betrachtet werden. Hierbei können sich Ausbildungsstand-abhängige Unterschiede zeigen, die sich ggf. auf die Befunde auswirken. Die Dokumentation erfolgte durch ein an die Schockraumbögen angehefteten Fragebogen, der sowohl von studentischem Hilfspersonal als auch von Ärzten der chirurgischen Notaufnahme ausgefüllt wurde. Eine personell vereinheitlichte Erhebung, Dokumentation, Auswertung und Untersuchungsdurchführung würde zu einer verbesserten Studienqualität führen, ist im Klinikalltag jedoch nur schwer realisierbar. Während des kompletten Untersuchungszeitraums wurde an der bildgebenden Apparatur nichts verändert, weshalb diesbezügliche Verfälschungen zu vernachlässigen sind. Als verbesserungswürdig könnte eine striktere und einheitlichere Durchführung aller drei Untersuchungsmodalitäten bei Schockraumpatienten mit Thoraxtrauma angedacht werden sowie ein längerer, prospektiver Untersuchungszeitraum, um mehr relevante Patienten zu detektieren. 
Tabelle 4.2: Autorenübersicht zur prozentualen Sensitivität von eFAST und Thoraxröntgenuntersuchung in der Pneumo-/Hämatothorax-Diagnostik.

\begin{tabular}{|c|c|c|c|c|}
\hline Autor & Jahr & $\begin{array}{l}\text { Patienten- } \\
\text { anzahl }\end{array}$ & \multicolumn{2}{|c|}{$\begin{array}{l}\text { Sensitivität der Pneumothorax- } \\
\text { /Hämatothorax-Diagnostik }\end{array}$} \\
\hline \multirow[t]{2}{*}{ Kirkpatrick et al. } & \multirow[t]{2}{*}{2004} & \multirow[t]{2}{*}{225} & eFAST & $49 \%$ \\
\hline & & & Thoraxröntgen & $20 \%$ \\
\hline \multirow[t]{2}{*}{ Zhang et al. } & \multirow[t]{2}{*}{2006} & \multirow[t]{2}{*}{135} & eFAST & $86,2 \%$ \\
\hline & & & Thoraxröntgen & $27,6 \%$ \\
\hline \multirow{2}{*}{$\begin{array}{l}\text { Nagarsheth und } \\
\text { Kurek }\end{array}$} & \multirow[t]{2}{*}{2011} & \multirow[t]{2}{*}{125} & eFAST & $81,8 \%$ \\
\hline & & & Thoraxröntgen & $31,8 \%$ \\
\hline Hyacinthe et al. & 2012 & 119 & $\begin{array}{l}\text { eFAST soll w } \\
\text { schneller Durcl } \\
\text { dynamisch un } \\
\text { Patienten angev }\end{array}$ & $\begin{array}{l}\text { r Sensitivität und } \\
\text { t auch bei hämo- } \\
\text { orisch instabilen } \\
\text { en. }\end{array}$ \\
\hline
\end{tabular}

Das eFAST ist der Thoraxröntgenuntersuchung in der Notfall-Diagnostik von Schwerverletzten im Schockraum mit einer vierfach höheren Sensitivität bei der Pneumo- und Hämatothorax-Diagnostik weit überlegen. Sie ist als aussagekräftigere Untersuchung anzusehen. Aufgrund des deutlichen Vorteils der Thoraxsonographie wird empfohlen, die Thoraxröntgenuntersuchung durch das eFAST im primären SchockraumAlgorithmus zur Pneumo-/Hämatothorax-Detektion bei Thoraxtraumen zu ersetzen. Darüber hinaus kann die höhere Detektionsrate des eFAST in Kombination mit der von Zhang et al. (2006) postulierten Zeitersparnis genutzt werden, um das klinische Schwerverletztenmanagement im Schockraum zu optimieren.

\subsubsection{Konsequenzen aus eFAST, Thoraxröntgenuntersuchung und CT}

Ein Pneumothorax kann über die aufgehobenen Adhäsionskräfte der Pleurablätter zum Kollabieren der betroffenen Lunge mit Einschränkung der Sauerstoffaustauschfläche führen. Die schwerwiegendste Form stellt der Ventil-/Spannungspneumothorax dar, der durch einen Überdruck in der Lunge zur Mediastinalverschiebung mit ggf. hämodynamischer Problematik führt (von Wichert 2004). Im Schockraum müssen Patienten mit einem Pneumothorax möglichst schnell und mit hoher Sensitivität erkannt 
werden, um lungenentlastende Maßnahmen mittels Thoraxdrainage einleiten zu können (Kopp et al. 2008).

In der hier vorgestellten Untersuchung konnte durch das eFAST mit sieben (64\%) der insgesamt elf entlastungswürdigen Befunde weitaus mehr pathologische Zustände diagnostiziert und direkt mittels Thoraxdrainage therapiert werden. Aus der Thoraxröntgenuntersuchung hingegen konnte laut Dokumentation keine einzige Konsequenz gezogen werden. Somit spiegeln sich auch in diesem Ergebnis der deutlich höhere Stellenwert und die höhere Aussagekraft des eFAST wider.

In den restlichen $36 \%$ der Fälle wurde die notwendige Konsequenz weder aus dem eFAST noch aus der Thoraxröntgenuntersuchung gezogen. Erst mithilfe des CT konnte die Pathologie erkannt und therapiert werden. Das CT stellt aufgrund der höchsten Sensitivität den Goldstandard in der Pneumothorax-Diagnostik dar und ist sogar dem eFAST überlegen (Reissig und Kroegel 2005).

Über die genauen Gründe, warum in 36\% der Fälle das eFAST die pathologischen Befunde nicht erkannt hat, lässt sich nur spekulieren. Dabei könnten eine variierende Anwenderexpertise, der Zeitdruck und situations-bedingter Stress im Schockraum oder eine Adipositas-bedingte verbreiterte thorakale Gewebsschicht eine fehlerprovozierende Rolle spielen. Der Zeitfaktor beeinflusst das Untersuchungsergebnis ebenfalls, da sich die Luftansammlung im Pleuraspalt mit zunehmender Behandlungszeit und durch umlagerungs-bedingte Manöver ggf. progredient darstellen kann.

Dies wäre ein interessanter Ansatzpunkt für weitere Untersuchungen, die sich unter zusätzlicher Berücksichtigung der zeitlichen Komponente mit auf den Pneumothorax auswirkenden Faktoren beschäftigt. Dabei könnte die Aussagekraft der Thoraxsonographie zu drei Schockraumzeitpunkten untersucht werden. Initial vor dem Umlagern auf die Schockraumtrage, direkt danach und anschließend nach dem zweiten Umlagern auf den CT-Tisch. Der Untersucher sollte dabei nicht gewechselt werden. Somit könnte eine Aussage zur zeitlichen und umlagerungs-bedingten Entwicklung eines Pneumothorax getätigt werden.

Auch Reissig und Kroegel (2005) konnten in einer Post-interventionsstudie zum Pneumothoraxausschluss den deutlichen Vorteil des eFAST hinsichtlich schnellerer, früherer und einfacherer Durchführung im Vergleich zur Thoraxröntgenuntersuchung zeigen. Die Thoraxröntgenuntersuchung ist dabei lediglich zur Einteilung der Größe des 
Pneumothorax sowie bei einer Diskrepanz zwischen klinischem- und eFAST Befund in Erwägung zu ziehen (Reissig und Kroegel 2005).

Sowohl bei der hier vorgestellten Studie als auch bei Reissig und Kroegel (2005) kann aufgrund eines positiven eFAST Befund mit großer Wahrscheinlichkeit von einem vorliegenden Pneumo-/Hämatothorax ausgegangen werden.

Bei negativem eFAST-Befund muss die im Vergleich deutlich niedrigere Sensitivität der Thoraxröntgenuntersuchung bedacht werden. Außerdem zeigt die von Hyacinthe et al. (2012) veröffentlichte Studie, dass die Kombination aus klinisch auffälligem Befund und Thoraxröntgenuntersuchung eine geringere Sensitivität aufweist als das eFAST alleine. Deshalb ist bei negativem eFAST mit allenfalls sehr geringer Wahrscheinlichkeit von einem widersprüchlichen Pneumothorax darstellenden Röntgenbefund auszugehen.

An der von Reissig und Kroegel (2005) durchgeführten Studie ist der nur gering zugunsten des eFAST ausfallende Sensitivitätsunterschied kritisch zu hinterfragen. Diese Tatsache ist der geringen Anzahl vorliegender Pneumothorax bzw. Hydropneumothorax geschuldet, die alle vier vom eFAST und nur einer von der Thoraxröntgenuntersuchung nicht erkannt wurde.

Dennoch unterstützen sowohl Reissig und Kroegel (2005) als auch Hyacinthe et al. (2012) die Feststellung der höheren Sensitivität des eFAST, verglichen mit der Thoraxröntgenuntersuchung. Somit spiegelt die deutlich höhere Konsequenzrate des eFAST indirekt auch deren höheren Stellenwert im Schockraummanagement zur Pneumothorax-Diagnostik wider.

Basierend auf dem Vorteil des eFAST zur initialen Schockraum- und postinterventionellen Pneumothorax-Diagnostik wäre eine weitergehende Untersuchung der Pneumothorax-Detektion nach zentraler Venenkatheter-Anlage interessant. Hierbei könnte nach Punktion der V. jugularis interna oder der V. subclavia die Aussagekraft der Thoraxsonographie zu definierten Zeitabschnitten post-interventionell untersucht werden.

Bei dem hier vorgestellten Studienablauf muss berücksichtigt werden, dass die Untersuchungen, Auswertungen und Dokumentation von eFAST, Thoraxröntgen und CT durch variierendes Personal der Unfallchirurgie bzw. Radiologie durchgeführt wurden. Eine personell standardisierte Erhebung, Dokumentation, Auswertung und Untersuchungsdurchführung würde zu einer verbesserten Studienqualität führen, ist im 
Klinikalltag jedoch nur schwer realisierbar. Während des kompletten Untersuchungszeitraums wurde an der bildgebenden Apparatur nichts verändert, weshalb Untersuchungsgerät-spezifische Abweichung $\mathrm{zu}$ vernachlässigen sind. Hinsichtlich der Anzahl detektierter Pneumo-/Hämatothorax gilt zu berücksichtigen, dass bei der Untersuchung insgesamt 20 Befunde festgestellt wurden. Davon wiesen nur elf ein entlastungwürdiges Ausmaß auf, sodass nur diese berücksichtigt wurden.

Das eFAST ist der Thoraxröntgenuntersuchung hinsichtlich der Detektion traumatisch und post-interventionell entstandener Pneumothorax in Sensitivität und der Einleitung notwendiger Maßnahmen deutlich überlegen. Deshalb ist das eFAST als initial wichtigste first look-Untersuchung im Schockraum zur Pneumothorax-Diagnostik bei Patienten mit Thoraxtrauma anzusehen.

\subsection{Stellenwert von Thoraxröntgenuntersuchung und cCT/Ganzkörper-CT bei verstorbenen Schockraumpatienten}

Um die Wertigkeit der verschiedenen diagnostischen Verfahren bei letztendlich letal schwerverletzten Patienten zu analysieren, wurden die bildgebenden Verfahren CT (cCT/Ganzkörper-CT) und Thoraxröntgenuntersuchung evaluiert. Die anschließende Gegenüberstellung der bildgebenden Verfahren mit den Daten des TraumaRegister DGUß zielt auf einen deutschlandweiten Vergleich strahlenbelastender Bildgebung bei schwerverletzten Schockraumpatienten ab.

Die festgestellte fast doppelt so hohe Prozentzahl durchgeführter cCT/Ganzkörper-CT Untersuchungen (76\%) im Vergleich zur Thoraxröntgenuntersuchung (40\%) spiegelt den deutlich höheren Stellenwert der Computertomographie bei verstorbenen Schockraumpatienten wider. Verglichen mit den Jahresberichtdaten des TraumaRegister DGU® (2017) und (2018) zeigt sich mit 34\% und 30\% durchgeführter Thoraxröntgenuntersuchung zu 78\% bzw. 79\% durchgeführter CT-Untersuchungen ebenfalls eine stärkere Präsenz des CT bei Schockraumpatienten.

Diese Feststellungen werden von den im 3-Jahres-Trend (2015 bis 2017) kontinuierlich steigenden Zahlen durchgeführter CT (77\% auf 79\%) und fallender Anzahl Thoraxröntgenuntersuchung (36\% auf 30\%) im Schockraum gestärkt (TraumaRegister DGU ${ }^{\circledR}$ 2017, TraumaRegister DGUß 2018). 
Auch die Gesamtzahl durchgeführter Bildgebungen der vergangenen 10 Jahre verdeutlicht mit 39\% Thoraxröntgenuntersuchung zu 76\% cCT/Ganzkörper-CT den insgesamt deutschlandweit höheren Stellenwert der Computertomographie im Schockraum (TraumaRegister DGUß 2018).

Kritisch muss hierbei betrachtete werden, dass es sich bei dem TraumaRegister DGU®Jahresbericht um ein Basis-Patientenkollektiv mit nicht nur verstorbenen Patienten handelt. Es werden auch überlebendende schwerverletzte Patienten mit einem MAIS 3+ sowie Patienten mit einem MAIS von 2, die auf Intensivstation gelegen haben, berücksichtigt, was die Vergleichbarkeit einschränkt. Dennoch handelt es sich sowohl bei den analysierten verstorbenen Schockraumpatienten als auch den Patienten des TraumaRegister DGUß um ein schwerverletztes Patientenkollektiv.

Der Vergleich durchgeführter bildgebender Verfahren hat ein deutlich zugunsten der Computertomographie ausfallendes Ergebnis gezeigt. Dank übereinstimmender Zahlen (Jahresbericht TraumaRegister DGU® (2018) und vorliegende Studie) kann die Aussage getätigt werden, dass das CT die deutschlandweit wichtigste Untersuchung bei schwerverletzten Schockraumpatienten ist.

Weiterhin zeigen CT-bezogene Studien wie die von Huber-Wagner et al. (2009), dass die Integration des Ganzkörper-CT in der frühen Traumaversorgung für eine signifikant höhere Überlebenswahrscheinlichkeit polytaumatisierter Patienten sorgt. Standardverfahren wie Sonographie oder Röntgen sollen deswegen nicht abgeschafft, sondern als Ergänzung bzw. Reserve im Falle eines CT Ausfalls verwendet werden (HuberWagner et al. 2009). Anhand dieser Empfehlung zeigt sich der deutlich höher einzuschätzende Stellenwert des CT im Schockraum verglichen mit anderen bildgebenden Verfahren.

In einer weiteren von Huber-Wagner et al. (2013) veröffentlichten Untersuchung konnte durch Anwendung des Ganzkörper-CT sowohl bei hämodynamisch instabilen als auch stabilen schwerverletzten Traumapatienten eine signifikante Senkung der standardisierten Mortalitätsrate (SMR) festgestellt werde. Damit ist die Anwendung des Ganzkörper-CT bei schwerverletzten Patienten eine sichere, durchführbare und gerechtfertigte Untersuchung (Huber-Wagner et al. 2013). Deshalb ist das CT als unverzichtbar in der Behandlung schwerverletzter Patienten anzusehen und ein Garant hoher diagnostischer Qualität im Schockraum. 
Aufgrund des höheren Stellenwertes der CT-Diagnostik und die favorisierte Durchführung im Schockraum muss auch die höhere Strahlenbelastung diskutiert werden. Das Ganzkörper-CT stellt mit einer Effektdosis zwischen 5 bis $20 \mathrm{mSv}$ im Vergleich zum konventionellen Röntgen (Wirbelsäule, Thorax, Becken) mit 5,46 mSv eine nahezu identische bis maximal vierfach höhere Strahlenbelastung dar (Brenner und Elliston 2004, Hara et al. 2009, Loewenhardt et al. 2012, Martinsen et al. 2012, Noël et al. 2013). Zusätzlich zeigt sich neben der vergleichbaren Strahlendosis und dem größeren Nutzen des Ganzkörper-CT bei polytraumatisierten Patienten auch eine signifikante Zeitverringerung, die bereits bei CT-Effektdosen von 10,2 bis $12,7 \mathrm{mSv}$ möglich ist (Heyer et al. 2005).

Der Zeitfaktor spielt in der Polytraumaversorgung eine wichtige Rolle, da bereits ab einem verlängerten Schockraumaufenthalt von 3 Minuten die Letalität um 1\% ansteigt (Clarke et al. 2002). Ein kürzerer Schockraumaufenthalt beeinflusst dabei die Intensivund Gesamtaufenthaltsdauer im Krankenhaus positiv, unabhängig von der Verletzungsschwere des Patienten (Prokop et al. 2006). Unter Berücksichtigung der verkürzten Diagnosedauer und besseren Darstellungsmöglichkeiten (3D Rekonstruktion) kann, laut Prokop et al. (2006), die einmalig höhere Strahlenbelastung des CT bei schwerverletzten Schockraumpatienten in Kauf genommen werden.

Es gilt zu beachten, dass bei den UMG-Daten ausschließlich im System registrierte und per Einsicht überprüfte Thoraxröntgenuntersuchung und cCT/Ganzkörper-CT berücksichtigt wurden. Beim Vergleichskollektiv des TraumaRegister DGU® (2017) und (2018) handelt es sich um gesammelte, handschriftlich dokumentierte Daten, die im Internet eingepflegt und nicht nochmals gegengeprüft wurden. Darüber hinaus muss berücksichtigt werden, dass die als Vergleichsgruppe gewählten bildgebenden Verfahren CT, entweder ein cCT oder ein Ganzkörper-CT beinhalten. Sollte ein Patient sowohl ein cCT als auch ein Ganzkörper-CT erhalten haben, so wurde dies als eine Untersuchung gewertet.

Der Vergleich von Thoraxröntgenuntersuchung und cCT/Ganzkörper-CT bei schwerverletzten und im Schockraum verstorbenen Patienten unterstreicht den hohen und deutschlandweit übertragbaren Stellenwert der CT-Diagnostik in der Schockraumbehandlung. Die hohe diagnostische Aussagekraft, der Zeitgewinn im Schockraumablauf und die letalitätssenkende Wirkung bei hämodynamisch instabilen Patienten sind Gründe, weshalb dem CT eine zentrale Rolle auch in der aktuellen Leitlinie Polytrauma 
(2016) zugesprochen wird (Huber-Wagner et al. 2013). Im aktuellen Schockraumalgorithmus stellt das CT den diagnostischen Goldstandard dar und ist in der Bildgebung schwerverletzter Patienten nicht wegzudenken (Leitlinie Polytrauma 2016).

\subsection{Fazit}

Die Verletzungsschwere des Thorax sowie eine durchgeführte Intubation oder Thoraxdrainage im Schockraum sind als richtungsweisende Faktoren einer notwendigen weiteren diagnostischen Abklärung von Mediastinalverletzungen und einer ggf. frühzeitig einzuleitenden Therapie anzusehen.

Die Analyse im Schockraum etablierter bildgebender Verfahren hat gezeigt, dass bei schwerverletzten Patienten die Thoraxröntgenuntersuchung weder zur Beurteilung der Mediastinalverhältnisse noch zur Pneumo-/Hämatothorax-Diagnostik eine relevante Aussagekraft besitzt und somit als nicht mehr zeitgemäß anzusehen ist.

Die deutlich höhere Sensitivität des eFAST zur Pneumo-/Hämatothorax-Diagnostik sowie die prozentual höhere Konsequenzrate, verglichen mit dem konventionellen Röntgen, bestätigen die deutliche Überlegenheit und den Vorteil der Thoraxsonographie bei der initialen Bildgebung von Thoraxtraumen im Schockraum.

Zusammenfassend ist eine Optimierung des Schockraummanagements bei Thoraxtraumen durch den initialen Einsatz des eFAST anstelle der Thoraxröntgenuntersuchung zur Pneumo-/Hämatothorax-Diagnostik möglich.

Bei der Gegenüberstellung von Thoraxröntgenuntersuchung und CT verstorbener und schwerverletzter Schockraumpatienten spiegeln die ermittelten und mit dem TraumaRegister DGU® verglichenen Daten eine höhere und tendenziell steigende Prozentzahl durchgeführter cCT/Ganzkörper-CT Untersuchungen wider. Deshalb wird das cCT/Ganzkörper-CT, auch laut aktueller Leitlinie Polytrauma (2016), als diagnostischer Goldstandard bei schwerverletzten Schockraumpatienten angesehen. 


\section{$5 \quad$ Zusammenfassung}

Die Relevanz bildgebender Verfahren im Schockraum bei Thoraxverletzungen wurde mit dem Ziel, eine möglichst hohe Qualität im Schockraummanagement zu gewährleisten, analysiert.

Die Aussagekraft der Thoraxröntgenuntersuchung zum Ausschluss mediastinaler Verletzungen wurde mittels retrospektiver Betrachtung der Jahre 2013 und 2014 analysiert. Das Mediastinum des gesamten Patientenkollektivs aus 267 Patienten wurde an zwei definierten Stellen (Herzklappenebene und Aortenbogen) ausgemessen und in Relation zum Thoraxdurchmesser gesetzt. Die Einteilung der Ergebnisse erfolgte anhand von vier definierter Grenzwerte $(\geq 0,2 / \geq 0,25 / \geq 0,28 / \geq 0,30)$. Die Testgenauigkeit wurde anschließend unter Berücksichtigung zweier Patientengruppen Auffällig (Patienten mit Mediastinalverletzung, $\mathrm{n}=27$ ) und Unauffällig (Patienten ohne Mediastinalverletzung, $n=240$ ) ermittelt. Eine maximale Sensitivität und Spezifität konnte auf Herzklappenebene (Mediastinum/Thorax-Quotient Messwert 1) bei einem Grenzwert von $\geq 0,3$ und auf Höhe des Aortenbogens (Mediastinum/Thorax-Quotient Messwert 2) bei $\geq 0,28$ ermittelt werden. Insgesamt zeigte sich jedoch keine ausreichend hohe und zufriedenstellende Testgenauigkeit. Deshalb hat die Thoraxröntgenuntersuchung hinsichtlich der Fragestellung pathologischer Verbreiterungen des Mediastinums in der Primärphase des Schwerverletztenmanagements im Schockraum an Aussagekraft und Relevanz verloren.

Zur Detektion prädiktiver Faktoren mediastinaler Verletzungen wurde das zuvor genannte Patientenkollektiv (Auffällig $\mathrm{n}=27$; Unauffällig $\mathrm{n}=240$ ) hinsichtlich patientenbezogener Daten, präklinisch und klinisch erhobener Parameter sowie Trauma-Scores und Letalität auf signifikante Unterschiede untersucht. Es zeigte sich ein signifikanter Unterschied bei zunehmender Thoraxverletzungsschwere, durchgeführter Intubation und Thoraxdrainage im Schockraum sowie Katecholamin-Gabe am Unfallort. Deshalb sollte bei diesen Parametern ein besonderes Augenmerk auf die weitere diagnostische Abklärung mediastinaler Verletzungen gelegt werden. Somit könnte der doppelt so hohen Letalität der Patienten mit mediastinaler Verletzung (Auffällig) durch eine frühzeitige Therapieeinleitung entgegengewirkt werden. 
Die Gegenüberstellung von eFAST und Thoraxröntgenuntersuchung zur Pneumothorax/Hämatothorax-Diagnostik zielt auf die Ermittlung der jeweiligen Testgenauigkeit ab. Es wurden prospektiv über zwölf Monate alle Schockraumpatienten, die eine Untersuchungskombination aus CT + eFAST und/oder Thoraxröntgenuntersuchung erhalten haben, analysiert. Dabei konnte gezeigt werden, dass mit einer Sensitivität von $80 \%$ das eFAST der konventionellen Thoraxröntgenuntersuchung mit 18\% weit überlegen und somit als valides und wichtigeres Untersuchungsmittel bei Thoraxtraumen anzusehen ist.

In einem zweiten Schritt wurde anhand des gleichen Patientenkollektivs die Anzahl direkt aufgrund vorangegangener Bildgebung eingeleitet invasiver Maßnahmen (Thoraxdrainage) untersucht. Es zeigte sich, dass das eFAST mit 64\% direkt initiierter Interventionen der Thoraxröntgenuntersuchung mit $0 \%$ eingeleiteter Maßnahmen deutlich überlegen ist.

Abschließend wurden in einer retrospektiven Erhebung der Jahre 2013 und 2014 alle noch im Schockraum oder spätestens 30 Tage nach Aufnahme über den Schockraum verstorbenen Patienten hinsichtlich der stattgefundenen strahlenbelastenden Bildgebung (cCT/Ganzkörper-CT und Thoraxröntgenuntersuchung) analysiert. Der anschließende Vergleich mit den deutschlandweit im TraumaRegister DGUß dokumentierten Schockraumpatienten zielt auf eine deutschlandweite Übertragbarkeit des Ergebnisses ab. Es zeigte sich, dass sowohl bei den analysierten 184 verstorbenen Schockraumpatienten als auch bei den vom Traumaregister DGUß (2018) dokumentierten Patienten das CT mit 76\% (Studie) bzw. 79\% (TraumaRegister) deutlich häufiger durchgeführt wurde als die Thoraxröntgenuntersuchungen mit 40 (Studie) bzw. 30\% (TraumaRegister). Somit wird der hohe und deutschlandweit übertragbare Stellenwert der CT-Diagnostik in der Schockraumbehandlung schwerverletzter Patienten zum Ausdruck gebracht. 


\section{$6 \quad$ Anhang}

6.1 Initiale Beurteilung des Mediastinums mittels Thoraxröntgenuntersuchung im Schockraum

Tabelle 6.1: Patientendaten und Messwerte aller analysierter Patienten $(\mathrm{n}=267)$.

\begin{tabular}{ll|ll}
\hline Auffällig: & Geschlecht & Unauffällig: & Geschlecht \\
\hline Männlich & $85 \%$ & Männlich & $77 \%$ \\
Weiblich & $15 \%$ & Weiblich & $23 \%$ \\
Ges.-Anzahl (n) & 27 & Ges.-Anzahl (n) & 240 \\
\hline Auffällig: & Alter & Unauffällig: & Alter \\
\hline Durchschnitt & $49 \mathrm{y}$ & Durchschnitt & $49 \mathrm{y}$ \\
Minimum & $16 \mathrm{y}$ & Minimum & $0 \mathrm{y}$ \\
Maximum & $89 \mathrm{y}$ & Maximum & $92 \mathrm{y}$ \\
1. Quantil & $26 \mathrm{y}$ & 1. Quantil & $33 \mathrm{y}$ \\
3. Quantil & $59 \mathrm{y}$ & 3. Quantil & $65 \mathrm{y}$ \\
Standardabweichung & $\pm 19,8$ & Standardabweichung & $\pm 21,2$ \\
Ges.-Anzahl (n) & 27 & Ges.-Anzahl (n) & 240 \\
\hline Auffällig: & 27 & Unauffällig: & Messwert 1 \\
\hline Durchschnitt & 0,302 & Durchschnitt & 0,298 \\
Minimum & 0,18 & Minimum & 0,18 \\
Maximum & 0,43 & Maximum & 0,52 \\
1. Quantil & 0,27 & Standardabweichung & $\pm 0,06$ \\
\hline
\end{tabular}




\begin{tabular}{ll|ll} 
Auffällig: & Messwert 2 & Unauffällig: & Messwert 2 \\
\hline Durchschnitt & 0,302 & Durchschnitt & 0,306 \\
Minimum & 0,21 & Minimum & 0,18 \\
Maximum & 0,47 & Maximum & 0,55 \\
1. Quantil & 0,27 & 1. Quantil & 0,26 \\
3. Quantil & 0,33 & 3. Quantil & 0,35 \\
Standardabweichung & $\pm 0,06$ & Standardabweichung & $\pm 0,06$ \\
Ges.-Anzahl (n) & 27 & Ges.-Anzahl (n) & 240 \\
\hline
\end{tabular}

Tabelle 6.2: Ergebnisverteilung der Grenzwerte des Mediastinum/Thorax-Quotienten 1.

\begin{tabular}{lllll} 
Grenzwert: & $\geq 0,2$ & $\geq 0,25$ & $\geq 0,28$ & $\geq 0,3$ \\
\hline Ges.-positiv & 258 & 208 & 155 & 103 \\
\hline Richtig positiv & 25 & 22 & 19 & 15 \\
Falsch positiv & 233 & 186 & 136 & 88 \\
& & & & 164 \\
\hline Ges.-negativ & 9 & 59 & 112 & 152 \\
\hline Richtig negativ & 7 & 54 & 104 & 12 \\
Falsch negativ & 2 & 5 & 8 & \\
\hline
\end{tabular}


Tabelle 6.3: Ergebnisverteilung der Grenzwerte des Mediastinum/Thorax-Quotienten 2.

\begin{tabular}{lllll}
\hline Grenzwert: & $\geq 0,2$ & $\geq 0,25$ & $\geq 0,28$ & $\geq 0,3$ \\
\hline Ges.-positiv & 266 & 223 & 165 & 125 \\
\hline Richtig positiv & 27 & 23 & 17 & 12 \\
Falsch positiv & 239 & 200 & 148 & 113 \\
\hline Ges.-negativ & 1 & & & 142 \\
\hline Richtig negativ & 1 & 44 & 102 & 127 \\
Falsch negativ & 0 & 40 & 92 & 15 \\
\hline
\end{tabular}

\subsection{Früherkennung mediastinaler Verletzungen}

Tabelle 6.4: Übersicht Herz/Thorax-Quotient und Throaxverletzungsschwere unterteilt in die Untersuchungsgruppen Auffällig und Unauffällig.

\begin{tabular}{ll|ll}
\hline Auffällig: & Herzsilhouette & Unauffällig: & Herzsilhouette \\
\hline Durchschnitt & $139,51 \mathrm{~mm}$ & Durchschnitt & $136,19 \mathrm{~mm}$ \\
Standardabweichung & $\pm 26,2$ & Standardabweichung & $\pm 23,5$ \\
Verbreitert & $22 \%$ & Verbreitert & $26 \%$ \\
Normal & $88 \%$ & Normal & $84 \%$ \\
Ges.-Anzahl (n) & 27 & Ges.-Anzahl (n) & 240 \\
\hline Auffällig: & Thoraxverletzung & Unauffällig: & Thoraxverletzung \\
\hline Keine & $0 \%$ & Keine & $49 \%$ \\
Leicht & $0 \%$ & Leicht & $18 \%$ \\
Mittel & $27 \%$ & Mittel & $24 \%$ \\
Schwer & $72 \%$ & Schwer & $9 \%$ \\
Ges.-Anzahl (n) & 11 & Ges.-Anzahl (n) & 194 \\
\hline
\end{tabular}


Tabelle 6.5: Übersicht der analysierten Verletzungsursachen unterteilt in die Untersuchungsgruppen Auffällig und Unauffällig.

\begin{tabular}{ll|ll}
\hline Auffällig: & Verletzungsursache & Unauffällig: & Verletzungsursache \\
\hline Verkehrsunfall & $67 \%$ & Verkehrsunfall & $61 \%$ \\
Sturz & $20 \%$ & Sturz & $35 \%$ \\
Sonstige & $13 \%$ & Sonstige & $4 \%$ \\
Ges.-Anzahl (n) & 15 & Ges.-Anzahl (n) & 205 \\
\hline
\end{tabular}

Tabelle 6.6: Vitalparameter Unfallort und Schockraum unterteilt in die Untersuchungsgruppen Auffällig und Unauffällig.

\begin{tabular}{ll|ll}
\hline Auffällig: & RR Unfallort & Unauffällig: & RR Unfallort \\
\hline Durchschnitt & $133 \mathrm{mmHg}$ & Durchschnitt & $134 \mathrm{mmHg}$ \\
Minimum & $60 \mathrm{mmHg}$ & Minimum & $75 \mathrm{mmHg}$ \\
Maximum & $190 \mathrm{mmHg}$ & Maximum & $220 \mathrm{mmHg}$ \\
1. Quantil & $124 \mathrm{mmHg}$ & 1. Quantil & $120 \mathrm{mmHg}$ \\
3. Quantil & $150 \mathrm{mmHg}$ & 3. Quantil & $150 \mathrm{mmHg}$ \\
Standardabweichung & $\pm 39,4$ & Standardabweichung & $\pm 25,3$ \\
Ges.-Anzahl (n) & 12 & Ges.-Anzahl (n) & 189 \\
\hline Auffällig: & $\mathrm{RR} \mathrm{Schockraum}$ & Unauffällig: & $\mathrm{RR} \mathrm{Schockraum}$ \\
\hline Durchschnitt & $122 \mathrm{mmHg}$ & Durchschnitt & $126 \mathrm{mmHg}$ \\
Minimum & $70 \mathrm{mmHg}$ & Minimum & $47 \mathrm{mmHg}$ \\
Maximum & $164 \mathrm{mmHg}$ & Maximum & $200 \mathrm{mmHg}$ \\
1. Quantil & $100 \mathrm{mmHg}$ & 1. Quantil & $109 \mathrm{mmHg}$ \\
3. Quantil & $158 \mathrm{mmHg}$ & 3. Quantil & $145 \mathrm{mmHg}$ \\
Standardabweichung & $\pm 32,6$ & Standardabweichung & $\pm 28,6$ \\
Ges.-Anzahl (n) & 14 & Ges.-Anzahl (n) & 196 \\
\hline
\end{tabular}




\begin{tabular}{|c|c|c|c|}
\hline Auffällig: & HF Unfallort & Unauffällig: & HF Unfallort \\
\hline Durchschnitt & $101 / \mathrm{min}$ & Durchschnitt & $88 / \mathrm{min}$ \\
\hline Minimum & $50 / \mathrm{min}$ & Minimum & $16 / \mathrm{min}$ \\
\hline Maximum & $166 / \mathrm{min}$ & Maximum & $199 / \mathrm{min}$ \\
\hline 1. Quantil & $80 / \mathrm{min}$ & 1. Quantil & $78 / \mathrm{min}$ \\
\hline 3. Quantil & $127 / \mathrm{min}$ & 3. Quantil & $100 / \mathrm{min}$ \\
\hline Standardabweichung & $\pm 33,2$ & Standardabweichung & $\pm 21,4$ \\
\hline Ges.-Anzahl (n) & 13 & Ges.-Anzahl (n) & 186 \\
\hline Auffällig: & HF Schockraum & Unauffällig: & HF Schockraum \\
\hline Durchschnitt & $90 / \mathrm{min}$ & Durchschnitt & $85 / \mathrm{min}$ \\
\hline Minimum & $18 / \mathrm{min}$ & Minimum & $18 / \mathrm{min}$ \\
\hline Maximum & $136 / \mathrm{min}$ & Maximum & $195 / \mathrm{min}$ \\
\hline 1. Quantil & $68 / \mathrm{min}$ & 1. Quantil & $72 / \mathrm{min}$ \\
\hline 3. Quantil & $100 / \mathrm{min}$ & 3. Quantil & $96 / \mathrm{min}$ \\
\hline Standardabweichung & $\pm 29,6$ & Standardabweichung & $\pm 21,6$ \\
\hline Ges.-Anzahl (n) & 14 & Ges.-Anzahl (n) & 198 \\
\hline Auffällig: & $\mathrm{SpO}_{2}$ Unfallort & Unauffällig: & $\mathrm{SpO}_{2}$ Unfallort \\
\hline Durchschnitt & $88 \%$ & Durchschnitt & $93 \%$ \\
\hline Minimum & $60 \%$ & Minimum & $30 \%$ \\
\hline Maximum & $100 \%$ & Maximum & $100 \%$ \\
\hline 1. Quantil & $85 \%$ & 1. Quantil & $92 \%$ \\
\hline 3. Quantil & $96 \%$ & 3. Quantil & $98 \%$ \\
\hline Standardabweichung & $\pm 11,2$ & Standardabweichung & $\pm 9,9$ \\
\hline Ges.-Anzahl (n) & 14 & Ges.-Anzahl (n) & 176 \\
\hline
\end{tabular}




\begin{tabular}{ll|ll}
\hline \multicolumn{3}{l}{ 6 Anhang } \\
\hline Auffällig: & $\mathrm{SpO}_{2}$ Schockraum & Unauffällig: & $\mathrm{SpO}_{2}$ Schockraum \\
\hline Durchschnitt & $95 \%$ & Durchschnitt & $96 \%$ \\
Minimum & $81 \%$ & Minimum & $35 \%$ \\
Maximum & $100 \%$ & Maximum & $100 \%$ \\
1. Quantil & $91 \%$ & 1. Quantil & $96 \%$ \\
3. Quantil & $99 \%$ & 3. Quantil & $100 \%$ \\
Standardabweichung & $\pm 5,7$ & Standardabweichung & $\pm 6,4$ \\
Ges.-Anzahl (n) & 12 & Ges.-Anzahl (n) & 181 \\
\hline
\end{tabular}

Tabelle 6.7: Polytrauma-Kennwerte unterteilt in die Untersuchungsgruppen Auffällig und Unauffällig.

\begin{tabular}{ll|ll}
\hline Auffällig: & NISS & Unauffällig: & NISS \\
\hline Durchschnitt & 39 Pkte. & Durchschnitt & 28 Pkte. \\
Minimum & 12 Pkte. & Minimum & 3 Pkte. \\
Maximum & 75 Pkte. & Maximum & 75 Pkte. \\
1. Quantil & 22 Pkte. & 1. Quantil & 17 Pkte. \\
3. Quantil & 50 Pkte. & 3. Quantil & 34 Pkte. \\
Standardabweichung & $\pm 18,6$ Pkte. & Standardabweichung & $\pm 15,1$ Pkte. \\
Ges.-Anzahl (n) & 15 & Ges.-Anzahl (n) & 206 \\
\hline Auffällig: & RISC-II-Score & Unauffällig: & RISC-II-Score \\
\hline Durchschnitt & $75 \%$ & Durchschnitt & $87 \%$ \\
Minimum & $14,2 \%$ & Minimum & $4,7 \%$ \\
Maximum & $99,8 \%$ & Maximum & $99,9 \%$ \\
1. Quantil & $35,2 \%$ & Standardabweichung & $\pm 22,1 \%$ \\
3. Quantil & $98,7 \%$ & Ges.-Anzahl (n) & 194 \\
Standardabweichung & $\pm 32,2 \%$ & $90,9 \%$ \\
Ges.-Anzahl (n) & 15 & $99,6 \%$ \\
\hline
\end{tabular}




\subsection{Letalität}

Tabelle 6.8: Letalität Schockraumpatienten unterteilt in die Untersuchungsgruppen Auffällig und Unauffällig.

\begin{tabular}{lc|l}
\hline Letalität: & Auffällig & Unauffällig: \\
\hline Männlich & 4 & 14 \\
Weiblich & 0 & 4 \\
Prozent & $15 \%$ & $18 \%$ \\
Durchschnittalter & $46,5 y$ & $65,8 \mathrm{y}$ \\
Minimum & 22 & 20 \\
Maximum & 63 & 89 \\
Standardabweichung & 18,4 & $\pm 23,8$ \\
Ges.-Anzahl (n) & 27 & 240 \\
\hline
\end{tabular}




\subsection{Fragebogen}

\section{Thoraxröntgenuntersuchung vs. eFAST}

Röntgenthorax durchgeführt

eFAST durchgeführt

eFAST nicht durchgeführt wegen Weichteilemphysem

eFAST Befunde:

Röntgen Befunde:

Unauffällig

Unauffällig

Hämatothorax

Hämatothorax

Pneumothorax

Pneumothorax

Andere:

Andere:

Vom eFAST divergierende Befunde:

Aufgrund von eFAST eingeleitete Maßnahmen:

Thoraxdrainage: $\quad$ Rechts $\square \quad$ Links

Andere/weitere Maßnahmen:

Bemerkungen:

Abbildung 6.1: Darstellung des angefertigten Fragebogens zur Primärdiagnostik eines Pneumo/Hämatothorax im Schockraum durch eFAST und Thoraxröntgenuntersuchung. 


\section{Literaturverzeichnis}

Achenbach T (2012): Röntgenthorax-Indikationen, Limitationen, Befundung. Lege Artis $2,258-263$

Adams H, Baumann G, Gänsslen A ud, Janssens U, Knoefel W, Koch T, Marx G, Müller-Werdan U, Pape H, Prange W, et al. (2001): Definitionen der Schockformen. Anasthesiol Intensivmed Notfallmed Schmerzther $\underline{38}, 140-143$

Albrecht T, von Schlippenbach J, Stahel P, Ertel W, Wolf K-J (2004): Die Rolle der Ganzkörper-Spiral-CT bei der Primärdiagnostik polytraumatisierter Patienten-Vergleich mit konventioneller Radiographie und Abdomensonographie. RöFo 176, 1142-1150

Baker SP, O’neill B (1976): The injury severity score: an update. J Trauma Acute Care Surg $\underline{16}, 882-885$

Baker SP, O’Neill B, Haddon Jr W, Long WB (1974): The injury severity score: a method for describing patients with multiple injuries and evaluating emergency care. $\mathrm{J}$ Trauma Acute Care Surg 14, 187-196

Balogh Z, Offner PJ, Moore EE, Biffl WL (2000): NISS predicts postinjury multiple organ failure better than the ISS. J Trauma Acute Care Surg $\underline{48}, 624-628$

Barcia TC, Livoni JP (1983): Indications for angiography in blunt thoracic trauma. Radiology $147,15-19$

Blackmore CC, Zweibel A, Mann FA (2000): Determining risk of traumatic aortic injury: how to optimize imaging strategy. Am J Roentgenol 174, 343-347

Blalock JB, Ochsner JL (1966): Management of thoracic trauma. Surg Clin North Am $\underline{46}, 1513-1524$

Boehm T, Alkadhi H, Schertler T, Baumert B, Roos J, Marincek B, Wildermuth S (2004): Einsatz der Mehrschicht-Spiral-CT beim traumatologischen Notfall und ihre Auswirkungen auf den Untersuchungs-und Behandlungsalgorithmus. RöFo 176, 17341742

Bölükbas S, Ghezel-Ahmadi D, Kwozalla AK, Schirren J (2011): Diagnostic assessment and treatment concepts for thoracic trauma. Chirurg $\underline{82}, 843-849$

Bouillon B, Rixen D, Maegele M, Steinhausen E, Tjardes T, Paffrath T (2009): Damage control orthopedics. Unfallchirurg $\underline{112}, 860-869$

Brambrink AM, Noppens R (2001): Der Notfallpatient im Schock. Notf Rett Med $\underline{4}, 4$ 15 
Brenneman FD, Boulanger BR, McLellan, Barry A, Redelmeier DA (1998): Measuring injury severitiy: times for a change. J Trauma Acute Care Surg 44, 580-582

Brenner DJ, Elliston CD (2004): Estimated radiation risks potentially associated with full-body CT screening. Radiology 232, 735-738

Butcher N, Balogh ZJ (2009): The definition of polytrauma: the need for international consensus. Injury $\underline{40}, 12-22$

Chawda M, Hildebrand F, Pape HC, Giannoudis PV (2004): Predicting outcome after multiple trauma: which scoring system. Injury $\underline{35}, 347-358$

Clarke JR, Trooskin SZ, Doshi PJ, Greenwald L, Mode CJ (2002): Time to laparotomy for intra-abdominal bleeding from trauma does affect survival for delays up to 90 minutes. J Trauma Acute Care Surg $\underline{52}, 420-425$

Copes WS, Champion HR, Sacco WJ, Lawnick MM, Keast SL, Bain LW (1988): The Injury Severity Score revisited. J Trauma Acute Care Surg 28, 69-77

Demetriades D, Velmahos GC, Scalea TM, Jurkovich GJ, Karmy-Jones R, Teixeira PG, Hemmila MR, O'Connor JV, McKenney MO, Moore FO, et al. (2008): Diagnosis and treatment of blunt thoracic aortic injuries: changing perspectives. J Trauma Acute Care Surg $\underline{64}, 1415-1419$

Diederich S, Wormanns D (2009): Radiologische Diagnostik der Pleura. Radiol Up2date $\underline{9}, 167-185$

Dokov W, Radoinova D (2014): Applicability of the abbreviated injury scale in forensic medical practice in Bulgaria. Scr Sci Med $\underline{46}, 51-54$

Doll D, Müller T, Ruchholtz S, Kühne CA (2010): Damage Control prioritätenorientiertes Behandlungsmanagement schwerstverletzter Patienten. Orthop Unfall Up2date $\underline{5}, 87-96$

Domej W, Haditsch B, Schaberger G (2011): Kritische Beurteilung der O2-Versorgung des Organismus durch Pulsoxymetrie. Alpinmed Rundbrief 44, 14-15

Encke A, Lüllig H, Ullrich F (1978): Das geschlossene und offene Thoraxtrauma. Unfallchirurgie $\underline{4}, 23-29$

Farkas ML: Querschnittanatomie zur Computertomographie. Springer, Berlin Heidelberg 1986, 3-9

Fischer M, Kehrberger E, Marung H, Moecke H, Prückner S, Trentzsch H, Urban B (2016): Eckpunktepapier 2016 zur notfallmedizinischen Versorgung der Bevölkerung in der Prähospitalphase und in der Klinik. Notf Rett Med 19, 387-395 
Flohr TG, Ohnesorge BM, Becker CR, Knez A, Reiser MF: Multi-slice and Dual-source CT in Cardiac Imaging. 2. Auflage; Springer, Berlin Heidelberg New York 2007, 41-69

Freeman P (1999): The role of ultrasound in the assessment of the trauma patient. Aust $\mathrm{J}$ Rural Health $\underline{7}, 85-89$

Freyschmidt J, Hahn D: Handbuch diagnostische Radiologie. Kardiovaskuläres System; Springer, Berlin Heidelberg 2007, 118

Frobenius H: Unfallmechanismen und ihre typischen Verletzungen. Fachkundenachweis Rettungsdienst; Springer, Berlin Heidelberg 1991, 189-192

Goldberg BB, Goodman GA, Clearfield HR (1970): Evaluation of ascites by ultrasound. Radiology 96, 15-22

Grau T: Ultraschall in der Anästhesie und Intensivmedizin. Lehrbuch der Ultraschalldiagnostik. Deutscher Ärzte-Verlag, Köln 2007, 79-80

Haasper C, Junge M, Ernstberger A, Brehme H, Hannawald L, Langer C, Nehmzow J, Otte D, Sander U, Krettek C, et al. (2010): Die Abbreviated Injury Scale (AIS). Unfallchirurg 113, 366-372

Hara AK, Paden RG, Silva AC, Kujak JL, Lawder HJ, Pavlicek W (2009): Iterative reconstruction technique for reducing body radiation dose at CT: feasibility study. Am $\mathrm{J}$ Roentgenol 193, 764-771

Heberer G (1968): Beurteilung und Behandlung von Verletzungen des Brustkorbes und der Brustorgane im Rahmen der Mehrfachverletzungen. Langenbecks Archiv für klinische Chirurgie $\underline{322}$, 268-284

Hehir M, Hollands M, Deane S (1990): The accuracy of the first chest x-ray in the trauma patient. Aust N Z J Surg $\underline{60}, 529-532$

Hermanutz K, Bücheler E (1974): Traumatische, nichtperforierende Verletzungen der thorakalen Aorta und der intrathorakalen Aortenbogenäste. RöFo 120, 156-163

Heyer CM, Rduch G, Kagel T, Lemburg SP, Theisinger A, Bauer TT, Muhr G, Nicolas V (2005): Prospektive, randomisierte Evaluation eines modifizierten Mehrdetektor-CTProtokolls in der Initialdiagnostik beim Polytrauma. RöFo 177, 242-249

Hinkelbein J, Faymonville C, Hackenbroch M, Burst V, Böttiger B (2013): Interdisziplinäre Schockraumversorgung polytraumatisierter Patienten. Notf Med Up2date $\underline{8}, 177-192$

Ho RT, Blackmore CC, Bloch RD, Hoffer EK, Mann FA, Stern EJ, Wilson AJ (2002): Can we rely on mediastinal widening on chest radiography to identify subjects with aortic injury. Emerg Radiol 9, 183-187

Hokema F, Donaubauer B, Busch T, Bouillon B, Kaisers U (2007): 
Schockraummanagement nach dem ATLS®-Algorithmus. Anästhesiol Intensivmed Notfallmed Schmerzther $\underline{42}, 716-723$

Huber-Wagner S, Lefering R, Qvick L-M, Körner M, Kay MV, Pfeifer K-J, Reiser M, Mutschler W, Kanz K-G, Society WG on P of the GT, et al. (2009): Effect of wholebody CT during trauma resuscitation on survival: a retrospective, multicentre study. Lancet $\underline{373}, 1455-1461$

Huber-Wagner S, Biberthaler P, Häberle S, Wierer M, Dobritz M, Rummeny E, van Griensven M, Kanz K-G, Lefering R, et al. (2013): Whole-body CT in haemodynamically unstable severely injured patients: a retrospective, multicentre study. PloS One $\underline{8}$, e68880

Hunink M, Bos JJ (1995): Triage of patients to angiography for detection of aortic rupture after blunt chest trauma: cost-effectiveness analysis of using CT. Am J Roentgenol 165, 27-36

Husum H, Strada G (2002): Injury severity score versus new injury severity score for penetrating injuries. Prehosp disast Med 17, 27-32

Hyacinthe A-C, Broux C, Francony G, Genty C, Bouzat P, Jacquot C, Albaladejo P, Ferretti GR, Bosson J-L, Payen J-F (2012): Diagnostic accuracy of ultrasonography in the acute assessment of common thoracic lesions after trauma. Chest $\underline{141}, 1177-1183$

Johansen K, Kohler TR, Nicholls SC, Zierler RE, Clowes AW, Kazmers A (1991): Ruptured abdominal aortic aneurysm: the Harborview experience. J Vasc Surg $\underline{13}$, 240247

Kanz KG, Eitel F, Waldner H, Schweiberer L (1994): Entwicklung von klinischen Algorithmen für die Qualitätssicherung in der Polytraumaversorgung. Unfallchirurg 97 , 303-307

Kanz K-G, Körner M, Linsenmaier U, Kay M, Huber-Wagner S, Kreimeier U, Pfeifer K-J, Reiser M, Mutschler W (2004): Prioritätenorientiertes Schockraummanagement unter Integration des Mehrschichtspiralcomputertomographen. Unfallchirurg 107, 937944

Keel M, Trentz O (2005): Pathophysiology of polytrauma. Injury $\underline{36}$, 691-709

Keßler U, Bergert H, Ockert D, Saeger HD (2002): Ergebnisse und Komplikationen in der Behandlung des rupturierten abdominellen Aortenaneurysmas. Zentralbl Chir $\underline{127}$, 664-668

Khandhar SJ, Johnson SB, Calhoon JH (2007): Overview of Thoracic Trauma in the United States. Thorac surg Clin $\underline{17}, 1-9$ 
Kirkpatrick AW, Sirois M, Laupland KB, Liu D, Rowan K, Ball CG, Hameed SM, Brown R, Simons R, Dulchavsky SA, et al. (2004): Hand-held thoracic sonography for detecting post-traumatic pneumothoraces: the Extended Focused Assessment with Sonography for Trauma (EFAST). J Trauma Acute Care Surg 57, 288-295

Kirschning T, Brenner F, Stier M, Weber CF, Walcher F (2009): Präklinische Notfallsonographie bei traumatologischen Patienten. Anaesthesist $\underline{58}, 51$

Kirsh MM, Behrendt DM, Orringer MB, Gago O, Gray Jr LA, Mills LJ, Walter JF, Sloan H (1976): The treatment of acute traumatic rupture of the aorta: a 10-year experience. An Surg 184, 308

Koerner M, Krotz MM, Degenhart C, Pfeifer K-J, Reiser MF, Linsenmaier U (2008): Current Role of Emergency US in Patients with Major Trauma 1. Radiographics 28, $225-242$

Kopp R, Andrassy J, Czerner S, Weidenhagen A, Weidenhagen R, Meimarakis G, Reiser M, Jauch K (2008): Traumatische thorakale Aortenruptur. Anaesthesist 57, 782

Körner M, Reiser M, Linsenmaier U (2009): Multidetektor-CT zur Diagnostik traumatologischen Notfälle. Radiologe $\underline{49}, 510-515$

Kram HB, Wohlmuth DA, Appel PL, Shoemaker WC (1987): Clinical and radiographic indications for aortography in blunt chest trauma. J Vasc Surg $\underline{6}, 168-176$

Kreuter M, Eberhardt R, Wenz H, Schmitteckert H, Heussel C, Herth F (2011): Diagnostische Wertigkeit der thorakalen Sonographie vergleichend zur Thoraxübersicht beim Nachweis eines postinterventionellen Pneumothorax. Eur J Ultrasound $\underline{32}$, E20E23

Kreuter M, Herth F, Wenz H, Mathis G (2012): Sonographische Notfalldiagnostik des Thorax. Pneumologe $\underline{9}, 436-443$

Lefering R (2002): Trauma Score Systems for Quality Assessment. Eur J Trauma 28, $52-63$

Lefering R (2009): Development and validation of the revised injury severity classification score for severely injured patients. Eur J Trauma Emerg Surg 35, 437-447

Lefering R: Abschätzung der Gesamtzahl Schwerstverletzter in Folge von Strassenverkehrsunfällen in Deutschland. Bundesanst Für Strassenwesen BASt 2010 http://www.dvr.de/download2/p2196/2196_1.pdf

Lefering R (2015): Ein neuer Prognose-Score im TraumaRegister DGUß(RISC II). Orthop Unfallchir $\underline{4}, 80-81$ 
Lefering R, Huber-Wagner S, Nienaber U, Maegele M, Bouillon B (2014): Update of the trauma risk adjustment model of the TraumaRegister DGU ${ }^{\mathrm{TM}}$ : the Revised Injury Severity Classification, version II. Crit Care $\underline{18}, 476$

Liener U, Sauerland S, Knöferl M, Bartl C, Riepl C, Kinzl L, Gebhard F (2006): Operative Versorgung von Thoraxverletzungen innerhalb der ersten Operationsphase. Unfallchirurg 109, 447-452

Lindemann H (1998): Respiratorische Insuffizienz und Sauerstofftherapie. Kinderheilkunde 146, 896-903

Lingawi SS, Buckley AR (2000): Focused Abdominal US in Patients with Trauma. Radiology 217, 426-429

Linsenmaier U, Schueller G (2010): Schockraum-Management. Notf Rett Med 13, 436444

Linsenmaier U, Kanz K-G, Mutschler W, Pfeifer K (2001): Radiologische Diagnostik beim Polytrauma: Interdisziplinäres Management. RöFo 173, 485-493

Loewenhardt B, Buhl M, Gries A, Greim C-A, Hellinger A, Hessmann M, Rathjen T, Reinert M, Manke C, Bernhard M (2012): Radiation exposure in whole-body computed tomography of multiple trauma patients: Bearing devices and patient positioning. Injury $\underline{43}, 67-72$

Marnocha K, Maglinte D, Woods J, Goodman M, Peterson P (1984): Mediastinalwidth/chest-width ratio in blunt chest trauma: a reappraisal. Am J Roentgenol 142, 275277

Marsh DG, Sturm JT (1976): Traumatic aortic rupture: roentgenographic indications for angiography. Ann Thorac Surg 21, 337-340

Martinsen ACT, Sæther HK, Hol PK, Olsen DR, Skaane P (2012): Iterative reconstruction reduces abdominal CT dose. Eur J Radiol 1ㅗ, 1483-1487

Mathis G, Osterwalder JJ: Notfallsonographie am Thorax. Bildatlas der Lungen-und Pleurasonographie; Springer, Berlin Heidelberg 2010, 249-253

Meier J, Habler O (2007): Die Behandlung auf der Intensivstation. Anasthesiol Intensivmed Notfallmed Schmerzther $\underline{42}, 724-730$

Messmer P, Jacob AL, Fries E, Gross T, Suhm N, Steinbrich W, Frede K, Schneider T, Regazzoni P (2001): Technologieintegration und Prozessmanagement Konzept und Implementierung einer neuartigen Plattform für einzeitige Diagnostik und Therapie des akut Kranken und Verletzten sowie für elektive computerassistierte Chirurgie (CAS). Unfallchirurg $\underline{104}, 1025-1030$ 
Michels G, Breitkreutz R, Pfister R (2014): Stellenwert der Lungensonographie in der Notfall-und Intensivmedizin. Deutsche Medizinische Wochenschrift 139, 2301-2307

Müller S, Bonatti J, Hellweg G, Pachinger O (2001): Erkrankungen der thorakalen Aorta: klinische Symptomatik, elektrokardiographische, röntgenologische und echokardiographische Diagnostik. Austrian J Cardiol $\underline{8}, 5-10$

Nagarsheth K, Kurek S (2011): Ultrasound detection of pneumothorax compared with chest X-ray and computed tomography scan. Am Surg 77, 480-484

Nast-Kolb D, Ruchholtz S, Oestern H-J, Neugebauer E (2000): Das Traumaregister der Arbeitsgemeinschaft Polytrauma der Deutschen Gesellschaft für Unfallchirurgie. Notfallmed Rettungsmed $\underline{3}, 147-150$

Neugebauer EA, Waydhas C, Lendemans S, Rixen D, Eikermann M, Pohlemann T (2012): The Treatment of Patients With Severe and Multiple Traumatic Injuries. Dtsch Arztebl Int 109, 102-108

Niethard FU, Pfeil J, Biberthaler P: Duale Reihe Orthopädie und Unfallchirurgie. Thieme Verlag, Stuttgart 2014, 339-343

Noël PB, Renger B, Fiebich M, Münzel D, Fingerle AA, Rummeny EJ, Dobritz M (2013): Does Iterative Reconstruction Lower CT Radiation Dose: Evaluation of 15,000 Examinations. PLoS one $\underline{8}$, e81141

Novelline RA, Rhea JT, Rao PM, Stuk JL (1999): Helical CT in Emergency Radiology. Radiology 213, 321-339

Osler T, Baker SP, Long W (1997): A modification of the injury severity score that both improves accuracy and simplifies scoring. J Trauma Acute Care Surg $\underline{43}$, 922-926

Pape H, Lefering R, Butcher N, Peitzman A, Leenen L, Marzi I, Lichte P, Josten C, Bouillon B, Schmucker U, et al. (2014): The definition of polytrauma revisited: An international consensus process and proposal of the new 'Berlin definition'. J Trauma Acute Care Surg 77, 780-786

Pape H-C, Tornetta P, Tarkin I, Tzioupis C, Sabeson V, Olson SA (2009): Timing of Fracture Fixation in Multitrauma Patients: The Role of Early Total Care and Damage Control Surgery. J Am Acad Orthop Surg 17, 541-549

Peters S, Heyer C, Lemburg S, Mohr P, Muhr G, Nicolas V (2007): MDCT-Diagnostik nichtvaskulärer Thoraxverletzungen beim Polytrauma. RöFo 179, 21

Piek J, Jantzen J-P (2000): Empfehlungen zur Erstversorgung des Patienten mit Schädel-Hirn-Trauma bei Mehrfachverletzung-Verabschiedet durch die DIVI am 5. November 1999. Op J $\underline{16}, 105-110$ 
Potthoff A, Gebel M, Rifai K (2012): Diagnostische und interventionelle Abdomensonographie. Internist $\underline{53}, 261-270$

Prokop A, Hötte H, Krüger K, Rehm KE, Isenberg J, Schiffer G (2006): MultisliceSpiral-CT zur Diagnostik beim Polytrauma. Unfallchirurg 109, 545-550

Raptopoulos V, Sheiman R, Phillips D, Davidoff A, Silva W (1992): Traumatic aortic tear: screening with chest CT. Radiology $\underline{182}$, 667-673

Regel G, Pape H-C: Lungenkontusion; in: Tscherne Unfallchirurgie; Springer, Berlin Heidelberg 2000, 251-272

Reissig A, Kroegel C (2005): Accuracy of transthoracic sonography in excluding postinterventional pneumothorax and hydropneumothorax: comparison to chest radiography. Eur J Radiol 53, 463-470

Reissig A, Kroegel C (2006): Sonographische Diagnostik des post-interventionellen Pneumothorax und Seropneumothorax-Prospektive Studie an 100 Patienten. Praxis $\underline{\text { 95, }}$ $617-624$

Reissig A, Copetti R, Kroegel C (2011): Current role of emergency ultrasound of the chest. Crit Care Med 39, 839-845

Rieger M, Sparr H, Esterhammer R, Fink C, Bale R, Czermak B, Jaschke W (2002): Moderne CT-Diagnostik des akuten Thorax- und Abdominaltraumas. Radiologe 42 , $556-563$

Rozycki GS, Shackford SR (1996): Ultrasound, what every trauma surgeon should know. J Trauma Acute Care Surg $\underline{40}, 1-4$

Ruchholtz S (2004): Das externe Qualitätsmanagement in der klinischen Schwerverletztenversorgung. Unfallchirurg 107, 835-843

Ruchholtz S, Zintl B, Nast-Kolb D, Waydhas C, Lewan U, Kanz K, Schwender D, Pfeifer K, Schweiberer L (1998): Improvement in the therapy of multiply injured patients by introduction of clinical management guidelines. Injury 29, 115-129

Ruchholtz S, Waydhas C, Schroeder T, Piepenbrink K, Kühl H, Nast-Kolb D (2002): Stellenwert der Computertomographie in der frühen klinischen Behandlung schwer verletzter Patienten. Chirurg $\underline{73}$, 1005-1012

Ruchholtz S, Kühne C, Siebert H, Weißbuch AU (2007): Das traumanetzwerk der deutschen gesellschaft für unfallchirurgie. Unfallchirurg $\underline{110}, 373-380$

Ruchholtz S, Lefering R, Paffrath T, Oestern HJ, Neugebauer E, Nast-Kolb D, Pape HC, Bouillon B (2008): Rückgang der Traumaletalität. Dtsch Arztebl 105, 225-231 
Schmitt K-U, Niederer PF, Muser MH, Walz F: Trauma-Biomechanik: Verletzungen in Straßenverkehr und Sport. Springer, Heidelberg Dordrecht London New York 2010, $30-32$

Schoepf U, Becker C, Brüning R, Hong C, Rust G-F, Helmberger T, Leimeister P, Stadie A, Niethammer M, Klingemann B, et al. (1999): Computertomographie des Abdomens mit der Mehrzeilen-Detektor Spiral-CT. Radiologe 39, 652-661

Schönenberger E, Mühler M, Dewey M (2010): Komplikationen durch die Kontrastmittelgabe. Internist $\underline{51}, 1516-1524$

Schramm P (2005): CT-Diagnostik bei akuter zerebraler Ischämie. Radiologe $\underline{45}, 420$ 429

Sefrin P, Appel E, Palm D: Sympatho-adrenerge Regulation in der Frühphase des traumatisch-hämorrhagischen Schocks. In: Brückner JB (Hrsg.): Kreislauf. Springer, Heidelberg New York 1980 217-221

Seltzer S, D'orsi C, Kirshner R, DeWeese J (1981): Traumatic aortic rupture: plain radiographic findings. Am J Roentgenol 137, 1011-1014

Shackford SR, Rogers FB, Osler TM, Trabulsy ME, Clauss DW, Vane DW (1999): Focused abdominal sonogram for trauma: the learning curve of non radiologist clinicians in detecting hemoperitoneum. J Trauma Acute Care Surg $\underline{46}$, 553-564

Spering C, Roessler M, Kurlemann T, Dresing K, Stürmer KM, Lehmann W, Sehmisch S (2018): Optimierte Ressourcenmobilisation und Versorgungsqualität Schwerstverletzter durch eine strukturierte Schockraumalarmierung. Unfallchirurg $\underline{121}, 1-8$ States JD, Viano DC (1990): Injury impairment and disability scales to assess the permanent consequences of trauma. Accident Anal Prev 22, 151-159

Storck T, Girwidz R (2016): Pulsoximetrie im Physikunterricht. Phys Did A 1, 1-13

Sturm J, Hoffmann R (2016): Die Akademie der Unfallchirurgie (AUC). Unfallchirurg $\underline{119}, 164-172$

Traub M, Stevenson M, McEvoy S, Briggs G, Lo SK, Leibman S, Joseph T (2007): The use of chest computed tomography versus chest X-ray in patients with major blunt trauma. Injury $\underline{38}, 43-47$

Oestern H-J, Trentz O, Uraneus S: Polytrauma: pathophysiology, priorities, and management. General Trauma Care and related Aspects; Springer, Berlin Heidelberg 2014, 69-76

Trupka A, Waydhas C, Nast-Kolb D, Schweiberer L (1995): Effect of early intubation on the reduction of post-traumatic organ failure. Unfallchirurg $\underline{98}, 111-117$ 
Tsai C-C, Tsai C-S, Ng K-K, Lai C-H, Hsueh S, Kao P-F, Chang T-C, Hong J-H, Yen T-C (2003): The impact of image fusion in resolving discrepant findings between FDGPET and MRI/CT in patients with gynaecological cancers. Eur J Nucl Med Mol Imaging $\underline{30}, 1674-1683$

Tscherne H, Regel G, Sturm JA, Friedl HP (1987): Degree of severity and priorities in multiple injuries. Chirurg $\underline{58}, 631-640$

Uffmann M, Fuchs M, Herold C (1998): Radiologie des Thoraxtraumas. Radiologe $\underline{38}$, 683-692

van Gestel AJR, Teschler H: Physiotherapie bei chronischen Atemwegs- und Lungenerkrankungen. Evidenzbasierte Praxis; Springer, Berlin Heidelberg 2015, 241242

von Ow D, Osterwalder J (2012): Sonographisches Schockraummanagement beim Trauma-Etabliertes Verfahren beim Traumamanagement. Klinikarzt $\underline{41}$, 23-27

von Wichert P (2004): Pneumothorax. Internist 4도 549-554

Wastl D, Helwig K, Dietrich CF (2015): Untersuchungskonzepte und Untersuchungsabläufe in der Notfallsonographie. Intensivmed Notfallmed 110, 231242

Waydhas C (2012): Präklinische polytraumaversorgung: S3-leitlinie. Unfallchirurg 115 , $8-13$

Whitaker IY, Gennari TD, Whitaker AL (2003): The difference between ISS and NISS in a series of trauma patients in Brazil. Assoc Adv Automot Med 47, 301-309

Woodring JH (1990): The normal mediastinum in blunt traumatic rupture of the thoracic aorta and brachiocephalic arteries. J Emerg Med $\underline{8}, 467-476$

Zehnder MA (1960): Unfallmechanismus und Unfallmechanik der Aortenruptur im geschlossenen Thoraxtrauma. Thoraxchirurgie $\underline{8}, 47-65$

Zehnder MA (1974): Unfallmechanismen und Unfallmechanik zur traumatischen Aortenruptur. Langenbecks Arch Surg 337, 325-328

Zhang M, Liu Z-H, Yang J-X, Gan J-X, Xu S-W, You X-D, Jiang G-Y (2006): Rapid detection of pneumothorax by ultrasonography in patients with multiple trauma. Crit Care $\underline{10}, \mathrm{R} 112$ 
Internetquellen:

Aschenbrenner I, Biberthaler P.: http:/www.dgu-online.de/en/patienteninformation/ haeufige-diagnosen/schwerverletzte/polytrauma.html; Zugriff am 21.07.2019

Auerbach K, Otte D, Jänsch M, Lefering R.: https://bast.opus.hbz-nrw.de/opus45bast/frontdoor/deliver/index/docId/277/file/medizinische_folgen_von_unfaellen.pdf; Zugriff am 28.07.2019

BAuA.: $\quad$ https://www.baua.de/DE/Themen/Arbeitswelt-und-Arbeitsschutz-imWandel/Arbeitsweltberichterstattung/Gesamtunfallgeschehen/Gesamtunfallgeschehen_n ode.html; Zugriff am 28.07.2019

Bundesärztekammer.: http://www.bundesaerztekammer.de/fileadmin/user_upload/ downloads/LeitRoentgen2008Korr2.pdf; Zugriff am 28.07.2019

TraumaRegister DGU®: http://www.traumaregister-dgu.de/fileadmin/user_upload/ traumaregister-dgu.de/docs/Downloads/TR-DGU-Jahresbericht_2017.pdf; Zugriff am 03.07.2019

TraumaRegister DGU®: http://www.traumaregister-dgu.de/fileadmin/user_upload/ traumaregister-dgu.de/docs/Downloads/TR-DGU-Jahresbericht_2018.pdf; Zugriff am 03.07.2019

DVR: https://www.dvr.de/download/beschluesse/2012-praezisierung-der-definition-derneuen-kategorie-lebensgefaehrlich-verletzt.pdf; Zugriff am 03.07.2019

Leitlinie Polytrauma: http://www.awmf.org/uploads/tx_szleitlinien/012-0191_S3_ Polytrauma_Schwerverletzten-Behandlung_2017-08.pdf; Zugriff am 28.07.2019

TeleKooperation TNW®: http://map.telekooperation-tnw.de/maps/\#zoom=14\&lat= 52.09878\&lon=8.89349\&layers=BTF; Zugriff am 03.07.2019

TraumaNetzwerk $\quad$ DGU®: $\quad$ http://www.traumanetzwerk-dgu.de/de/auditierung zertifizierung.html; Zugriff am 03.07.2019a

TraumaNetzwerk DGU®: http://www.traumanetzwerk-dgu.de/de/startseite_tnw.html; Zugriff am 03.07.2019b

UMG: http://cuop-umg.de/klinik/schwerpunkte/polytrauma; Zugriff am 03.07.2019

Weißbuch Schwerverletzten-Versorgung DGU®: http://www.dgu-online.de/uploads/ media/DGU_Weissbuch_A4_Internetendgueltige_version_1_.pdf; Zugriff am 28.07.2019 


\section{Danksagung}

Ein besonderer Dank gilt meinem Doktorvater Herrn Prof. Dr. med. Klaus Dresing für die Überlassung des Dissertationsthemas sowie für den geduldigen Umgang und die Unterstützung während des gesamten Entstehungs- und Entwicklungsprozesses der hier veröffentlichten Arbeit.

Bedanken möchte ich mich auch bei Herrn Dr. med. Christopher Spering. Durch dessen Engagement zwei Ergebnispräsentationen (2016 und 2017) auf dem Deutschen Kongress für Orthopädie und Unfallchirurgie in Berlin realisiert werden konnten. 


\section{Curriculum vitae}

Am 28. Januar 1990 wurde ich als Sohn von Heike Rapp-Brauns und Dr. Dirk Sven Arthur Brauns in Kehl am Rhein geboren. Den deutsch-französischen Kindergarten besuchte ich in Kehl am Rhein. Die Grundschule in Leutesheim und von 2000 bis 2009 das Einstein-Gymnasium in Kehl. Im Juni 2009 erlangte ich dort das Abitur.

Während des Wehrersatzdienstes 2009/2010 arbeitete ich zunächst als Rettungshelfer, anschließend von 2010 bis 2017 nach abgeschlossener Rettungssanitäter-Ausbildung beim Deutschen Roten Kreuz, Rettungsdienst Ortenau gGmbH.

2010 begann ich mit dem Studium der Zahnmedizin an der Universität Leipzig bis 2012 und absolvierte dort 2011 die Naturwissenschaftliche Vorprüfung. Im Jahr 2012 erfolgte der Studium- und Fakultätswechsel zur Humanmedizin an die Georg-AugustUniversität Göttingen. Während des Studiums arbeitete ich im studentischen Rufdienst der Unfallchirurgie der Universitätsmedizin Göttingen sowie im Rufdienst der Unfallund Viszeralchirurgie des Evangelischen Krankenhauses Göttingen-Weende. Im Jahr 2015 erhielt ich ein Stipendium der Deutschen Gesellschaft für Orthopädie und Unfallchirurgie für die Berliner Jahrestagung. Während des Praktischen Jahres sammelte ich Erfahrungen sowohl in Häusern der Maximalversorgung in Deutschland als auch grenzüberschreitend in der Schweiz.

Im November 2017 wurde ich als Arzt approbiert und arbeite seit Januar 2018 als Assistenzarzt im Klinikverbund SRRWS Spital Altstätten, Abteilung Orthopädie. 